\title{
Cytokines and Depression: A neurochemical hypothesis
}

Citation for published version (APA):

Bonaccorso, S. (2005). Cytokines and Depression: A neurochemical hypothesis. [Doctoral Thesis, Maastricht University]. https://doi.org/10.26481/dis.20050126sb

Document status and date:

Published: 01/01/2005

DOI:

10.26481/dis.20050126sb

Document Version:

Publisher's PDF, also known as Version of record

\section{Please check the document version of this publication:}

- A submitted manuscript is the version of the article upon submission and before peer-review. There can be important differences between the submitted version and the official published version of record.

People interested in the research are advised to contact the author for the final version of the publication, or visit the DOI to the publisher's website.

- The final author version and the galley proof are versions of the publication after peer review.

- The final published version features the final layout of the paper including the volume, issue and page numbers.

Link to publication

\footnotetext{
General rights rights.

- You may freely distribute the URL identifying the publication in the public portal. please follow below link for the End User Agreement:

www.umlib.nl/taverne-license

Take down policy

If you believe that this document breaches copyright please contact us at:

repository@maastrichtuniversity.nl

providing details and we will investigate your claim.
}

Copyright and moral rights for the publications made accessible in the public portal are retained by the authors and/or other copyright owners and it is a condition of accessing publications that users recognise and abide by the legal requirements associated with these

- Users may download and print one copy of any publication from the public portal for the purpose of private study or research.

- You may not further distribute the material or use it for any profit-making activity or commercial gain

If the publication is distributed under the terms of Article $25 \mathrm{fa}$ of the Dutch Copyright Act, indicated by the "Taverne" license above, 
A Bart 


\section{Contents}

Introduction

Chapter I: Behavioral changes in rats after acute, chronic and repeated administration of interleukin-1beta: relevance for affective disorders.

Chapter II: Immune markers in fibromyalgia: comparison with major depressed patients and normal volunteers.

Chapter III: Serotonin-immune interactions in elderly volunteers and in patients with Alzheimer's disease (DAT): lower plasma tryptophan availability to the brain in the elderly and increased serum interleukin- 6 in DAT.

Chapter IV: Psychological and behavioural effects of IFN-alpha in patients with hepatitis chronic $\mathrm{C}$.

Chapter V: Immunotherapy with interferon-alpha in patients affected by chronic hepatitis $C$ induces an intercorrelated stimulation of the cytokine network and an increase in depressive and anxiety symptoms.

Chapter VI: Increased Depressive Ratings in Patients With Hepatitis C Receiving Interferon-alpha-Based Immunotherapy Are Related to Interferon-alpha-Induced Changes in the Serotonergic System.

Chapter VII: Treatment with interferon-alpha (IFN alpha) of hepatitis C patients induces lower serum dipeptidyl peptidase IV activity, which is related to IFN alpha-induced depressive and anxiety symptoms and immune activation.

Chapter VIII: Lower activities of serum peptidases predict higher depressive and anxiety levels following interferon-alpha-based immunotherapy in patients with hepatitis $C$.

Chapter IX: Depression induced by treatment with interferon-alpha in patients affected by hepatitis $C$ virus. 
4 Cytokines and Depression: A Neurochemical Hypothesis 


\title{
Cytokines and Depression:
}

\section{A Neurochemical Hypothesis}

\author{
Proefschrift \\ ter verkrijging van de graad van doctor aan de Universiteit Maastricht \\ op gezag van de Rector Magnificus, Prof. mr. G. P. M. F. Mols, \\ volgens het besluit van het College van Decanen in het openbaar te verdedigen \\ op woensdag 26 januari 2005 om 12.00 uur \\ door \\ Stefania Bonaccorso \\ geboren op 6 October 1970 te Rome (Italie) \\ Promotores \\ Prof. dr. M. Maes \\ Beoordelingscommissie \\ Prof. dr. E.J.L. Griez (voorzitter) \\ Dr. M. de Baets \\ Dr. N. Nicolson \\ Prof. dr. S. Scharpè, Universiteit van Antwerpen, B. \\ The publication of this thesis was financially supported by \\ 'Het Van Leersumfonds' of the Royal Dutch Academy of Sciences (KNAW).
}




\section{Introduction}

\section{Cytokines and Depression: a neurochemical hypothesis}

\section{Dr. Stefania Bonaccorso M. D.}

Major Depression is a common psychiatric disorder, recently defined by the DSM-IV-TR (DSM-IV-TR, 2001) as a period of at least two weeks during which are present somatic and psychological symptoms like depressed mood, loss of interest or pleasure in usual activities, loss of weight or appetite, sleep disturbances, enhanced or lowered psychomotor activity, anergy, guilty feelings, difficulties in concentration, and suicidal thoughts. Different factors have been thought to be responsible for its etiology: genetic factors, heritable patterns (temperament), and emotional states to life events. More recently the positive results obtained by the administration of psychodrugs, normally used to treat major depressive episodes, pointed out the importance of the monoamine hypothesis (Delgado et al., 1990). This theory involves a dysfunction of the noradrenergic, dopaminergic and serotonin systems. Recently, the hypothesis of catecholamines depletion initially proposed by Bunney (Bunney et al., 1965) in the 6o's has been substituted by the idea of a catecholamine's dysregulation according to which a negative feedback on presynaptic neurons causes an exaggerated norepinephrine release. Depressed patients have shown higher plasma norepinephrine (NE) levels (Maes et al., 1991), in urine, blood and cerebrospinal fluid (Maes et al, 1999). Further studies showed a sub sensitivity of alpha2-ARs receptors which might be intended as the cause of the negative feedback on the presynaptic catecholaminergic neurons, and therefore responsible for the disinhibition of noradrenergic output in response to any activation (Kafka et al, 1986). Moreover, noradrenergic projections to the hippocampus have recently been implicated in behavioral sensitization to stress, and prolonged activation of the locus coeruleus contributes to the state of learned helplessness, an animal model for depression (Thase, 2000). Thus a dysregulation of NE system might contribute to the pathophysiology of depression.

Another neurotransmitter suspected to be involved in the etiology of depression is serotonin $(5-\mathrm{HT})$ : serotonin system is widely distributed in the brain and also its innervations. Serotonergic neurons projecting to the suprachiasmatic nucleus of the hypothalamus help to regulate circadian rhythms like sleep-wake cycles, body temperature, and hypothalamic-pituitary-adrenocortical axis function. Serotonin also permits or facilitates goal-directed motor and consummatory behaviors in conjunction with norepinephrine and dopamine (Thase, 2000). Dysfunctions in the serotonin metabolism (Maes \& Meltzer, 1995) have been documented in depressed people, for example, Maes and coworkers found lower levels of tryptophan (TRP), (the 5-HT precursor which compete with other competing amino acids (CAA) for entrance through the blood-brain barrier) along with a lower Trp/CAA ratio (Maes, 1996). The depletion of plasma concentration of tryptophan is likely to reduce the serotonin synthesis in the brain since this latter depends on the plasma availability of tryptophan. In support of this observation, dietary depletion of L-tryptophan will induce this abnormality in a subset of vulnerable patients. Tryptophan depletion also reverses acute responses to selective serotonin 
reuptake inhibitors and therefore the therapeutic effects of antidepressant medication (Dursun et al., 2001).

Alterations of the serotonergic system have been found also in subsets of depressed patients who showed a state of a relative hypofrontality of cerebral blood flow and glucose metabolism fully consistent with decreased neurotransmission by 5-HT neurons projecting from the dorsal raphe nuclei (Mayberg, 1997). Moreover in depressed patients it has been noticed also changes in 5-HT2 and 5-HT1A brain receptors (Maes \& Meltzer, 1995) in fact increased or lowered receptors binding, respectively for $5-\mathrm{HT}_{2}$ and $5-\mathrm{HT} 1 \mathrm{~A}$ receptors, have been found in various brain regions like frontal, temporal, limbic cortex of medicated or unmedicated patients. (Yatham et al., 2000; Sargent et al., 2000). Moreover changes in the 5$\mathrm{HT}$ transporter ( $5-\mathrm{HTT}$ ), a molecule located on the presynaptic membrane of $5-\mathrm{HT}$ cell bodies in the raphe nuclei that regulates 5 -HT uptake levels in the synaptic cleft, have been correlated to the pathophysiology of mood disturbances (Lesch et al., 1996). This modulation seems to be genetically mediated; in fact, the polymorphism at the level of the gene responsible for the 5 -HT variation seems to be related to anxiety-, depression- and aggression-related personality traits and therefore it seems to influence the risk of affective disorders (Lesch et al., 1998). All the alterations and dysfunction of the serotonin system as described above interact and participate at different levels and degrees to the pathophysiology of depression (Wichers et al., 2002).

Dopamine is the other neurotransmitter possibly involved in the etiology of mood disturbances: in fact, decreases in mesocortical and mesolimbic dopamine activity have implications in the cognitive, motor, and hedonic disturbances associated with depression. Moreover, dopamine activity appears to be potentiated by nicotinic inputs and glucocorticoids, and its concentrations seem to be correlated with brain serotonin activity and therefore with all the alterations citated above (Kapur et al., 1992).

We may conclude that, after nearly 30 years of research, depressed people manifest one or more abnormalities of monoamine neurotransmission (Cooper et al., 1996). This hypothesis needs to be modulated on the results obtained researching the neuroendocrine system (Holsboer, 1995): its alterations, interactions and correlations with the monoaminergic system constitutes another hypothesis for the pathophysiology of major depression.

This idea is drawn by the observation that stressful experiences may induce elevated glucocorticoids activity and from the knowledge that hypercortisolism is one of the most common correlates of melancholic depression. Corticotropin-releasing factor (CRF) is synthesized, during stress, in the paraventricular nucleus (PVN) and released from its terminals, stimulating the anterior pituitary gland, which releases adrenocorticotropin hormone (ACTH), which in turn induces the release of glucocorticoids from adrenal cortex (Arborelius et al., 1999). Cortisol secretion is increased in 20 to 40 percent of depressed outpatients and 40 to 60 percent of depressed inpatients with higher rates among older patients, particularly those with highly recurrent or psychotic depressive disorders. This evidence leads to the assumption that stress is somehow related to the developing of major depressive symptoms. Starvation and protracted sleep deprivation can also induce hypercortisolism. Acute stress is also correlated to transient serotonin release while chronic stress seems to be responsible for serotonin depletion (Fujino et al. 2002; Cassano \& D'mello, 2001; Kitayama et al. 1989). The synthesis of 5-HT1A auto receptors in the dorsal raphe nucleus during chronic stress may lead to a 
decreased serotonin transmission, even though elevations of glucocorticoids levels seem to induce an enhanced serotonin transmission as a compensatory effect, at least in the initial phases of stress. Moreover, a deficit of 5-HT activity and an increase in norepinephrine or acetylcholine activity have been shown to increase cortical-hypothalamic-pituitary-adrenal cortical activity (Thase, 2000). These neurochemical changes have been seen to produce in animals' behavioral changes such as suppression of exploratory behavior, decrease of food intake and sexual behavior, increase of conflict behavior and grooming (Dunn et al., 1990; Owens et al., 1991).

In humans, depressed people may show a non-suppression of cortisol secretion following administration of the synthetic glucocorticoid, dexamethasone. This is one of the most common tests used to demonstrate a dysfunction of hypothalamic-pituitary-adrenal axis in major depression. The partial non-suppression of cortisol in depressed patients can indicate a premature loss of inhibitory hippocampal glucocorticoid receptors. Therefore it has been postulated that early trauma, intended as early stressful life events, could elicit an early hypercortisolism which in turn may result into a disruption of those neurons and therefore be involved in a predisposition to the development of depression or in its pathophysiology (Thase, 2000).

There is now growing evidence that there are links between the immune system and the central nervous system (CNS). These reciprocal interactions highlight the importance of an active area of investigation that may achieve new insights in the etiology of neuropsychiatric disorders.

The immune system protects the body from pathogens either viruses, bacteria, fungi, parasites or neoplastic cells. It is divided into a lymphoid section (that leads to the formation of B-, T-lymphocytes and natural killer cells) and a myeloid section (that leads to the immunocomptence of blood cells such as monocytes, granulocytes, neutrophils, eosinophils, basophils with further differentiation for monocytes and basophils into macrophages and mast cells).

Macrophages, microglia, endothelial and reticular cells, natural killer cells along with the macrophages-derived cytokines (such as interferon-alpha, -beta, tumor necrosis factor-alpha) act against pathogens in a non-specific manner according to the so called natural immunity. B- and T-lymphocytes together with lymphocytes-derived cytokines such as interferongamma mediate the specific immunity. This latter immune pathway act with the aid of the major histocompatibility complex (MHC) which is in charge to present pathogens or neoplastically transformed cells to B- or T-lymphocytes. Most cells normally express MHC molecules type I while cells of immune origin normally also express $\mathrm{MHC}$ molecules type II. Some T cells recognize antigens only when associated with one or the other MHC types. The T-lymphocytes that recognize antigens associated with the MHC type I normally express on the surface a glycoprotein that is referred to a cluster differentiation, named CD8 while the T-lymphocytes that recognize antigens associated to the MHC type II present a glycoprotein named CD4.

The CD4 T-lymphocytes classes II MHC are normally called T-helper for the ability to secrete cytokines after activation. The profile of cytokines that they can secrete is different according to the different T-helper sub-populations. The T-helper1 (Th1) are normally in charge for the secretion of the so-called pro-inflammatory cytokines such as interleukin-2 (IL-2), interferongamma (IFN-gamma) normally involved in cell mediated inflammatory reaction with a final 
result of an activation of cytotoxic cells. T-helper2 (Th2) instead secretes interleukin-4 (IL-4), interleukin-5 (IL-5), and interleukin-10 (IL-10) normally known as anti-inflammatory cytokines (Miller et al., 2000).

Cytokines are polypeptides normally produced by different immunocytes in response to several stimuli, either physiological or pathological. They regulate the growth and function of immune cells when activated by an antigen or an infection but they also represent a way to communicate not only between immunocytes but also between other cells as well, in an autocrine or paracrine fashion; in fact, cytokine receptors are found in the periphery and the central nervous system such as hypothalamus, limbic system, brain cortex, hippocampus (Black, 1994). The evidence for catecholamines, serotonergic, cholinergic receptors on immune cells (Westly et al., 1987; Madden et al., 1991; Titinchi at al., 1984; Janowsky et al., 1986; Bonnet et al., 1987; Martelletti et al., 1987; Silvermann et al., 1986) seems to indicate a bi-directional communication between central nervous system and immune system. It has been questioned whether cytokines could pass the blood-brain-barrier (BBB) which normally restricts the exchange of molecules between plasma and extracellular fluids of the brain. There are different hypothesis:

1) cytokines can pass the blood brain barrier where the barrier is weaker, for example at the level of organum vascolosum laminae terminalis (OVLT) or at the preoptic area (Stitt et al., 1985; Nakamori et al., 1993): there is evidence of a transmission of cytokine signals such as interleukin-1 (IL-1) along sensory afferents: in fact, IL-1 $\beta$ peripheral production has been shown to activate subdiaphragmatic vagal afferents (Maier et al., 1998). This activation elicits the transportation of signals in the nucleus tractus solitarius (NTS) and in the area postrema where the vagal afferents terminate. In turn, NTS projects to the hippocampus and hypothalamus acting on central IL-1 receptors. This interaction seems to provoke a central induction of IL-1 produced by astrocytes, microglia and neurons, in charge also for the production of others cytokines as well. In fact, an experiment carried out by Ericson et al. (1994) showed that systemic administration of IL-1 induces an activation of the neurons of the nucleus of solitary tract that receives vagal afferents. Instead, in the rat, a vagotomy can suppress the pyrogenic effects of IL-1 injected intraperitoneally (Watkins et al., 1995). These evidences taken together seem to describe, during inflammation, a peripheral activation of vagal afferents by IL-1.

2) A third hypothesis is that T and B-lymphocytes can pass the blood brain barrier, during inflammation or injury, through the induction of adhesion molecules such as ICAM and VCAM-1 in the brain endothelium. This induction seems to increase the potential for circulating T lymphocytes, especially CD4 lymphocytes (Brown, 2001; Raivich et al., 1998; Sun et al., 1999; Tipold et al., 2001 Hickey, 2001).

3) Another hypothesis is that the production of prostaglandins (PGE) and nitric oxide (NO) normally elicited by the secretion of cytokines may alter the blood brain barrier permeability as well and allow the passage of cytokines (Boje et al., 2003).

The hypothesis that major depression is related to an activation of the inflammatory response system (IRS) is drawn by the clinical findings, which observed several immune alterations and endocrine disorders associated with depressive symptoms. 
Several authors gave essential scientific contributions to this matter.

Maes et al. (1990) first described an activation of the inflammatory response system (IRS), as well as an activation of some aspects of cell-mediated immunity (Maes 1997, 1999) during major depression. These findings can be summarized as follows:

1) The presence of an acute phase response of the IRS is indicated by the increased serum concentrations of positive phase reactants, such as haptoglobin, C- reactive protein, and alpha1-antitrypsin (Maes 1997, 1999, 1993; Song et al., 1994; Sluzewska et al., 1996). It can be observed also a decreased serum concentrations of negative acute phase reactants such as albumin, transferrin and zinc (Maes 1997, 1999; 1993);

2) The presence of an activation of monocytes and T-lymphocytes (and specific subsets) is demonstrated by an increased serum, urine, or cerebrospinal fluid (CSF) products like neopterin and prostaglandin E2 (PGE2). Neopterin is a marker of cell-mediated immunity found in serum and urine of depressed patients (Bonaccorso et al., 1998) along with PGE2 which has been found also in CSF or culture supernatant obtained after polyclonal stimulation of whole blood (Song et al., 1998);

3) The presence of an activation of monocytes and T-lymphocytes (Th1 subset) is also indicated by increased serum concentration and increased production of monocytes and Th1-like lymphocitic cytokines or cytokine receptors or cytokine receptor antagonists, such as interleukin 1beta (IL-1beta), interleukin-2 (IL-2), interleukin-6 (IL-6), interferongamma (IFN-gamma), the soluble IL- 6 receptor (IL-6R) and the IL-1R antagonist (IL-1RA) (Maes 1991, 1997, 1999).

An unbalance between the so called pro-inflammatory cytokines (IL-1, IL-2, IFN-gamma, IL6 , TNF-alpha) (Maes 1993, 1994) produced by the monocytes and Th1 lymphocytes subset and the anti-inflammatory cytokines (IL-4, IL-5, IL-10) mainly produced by the Th2 lymphocytes subset may cause changes in neurotransmission and neuroendocrine system and therefore produce depressive symptoms (Maes \& Smith, 1995; Smith, 1991).

Different cytokines seem to be responsible for the complex interaction between the immune system, endocrine function, brain and behavior. Moreover, they seem to play a fundamental role in different levels, such as on serotonin, dopamine and norepinephrine neurotransmission and on the hypothalamic-pituitary-adrenal axis.

Interleukin-1beta given intraventricularly (i.v.) activates the corticotropin releasing factor (CRF) and induces ACTH secretion (Matta et al., 1990). The activation of the hypothalamicpituitary-adrenal axis (HPA) by IL-1 seems to be mediated by prostaglandin PGE2, which in turn activates the neurons of PVN, which contain corticotropin-releasing hormone. HPA is also activated by the noradrenergic neurons in the cerebral area $A 1$ that projects to the neuroendocrine PVN (Konsman et al., 2002). The activation of noradrenergic neurons seems to be promoted also by IL-1 (see next paragraph). No difference are noticed when IL-1 is injected intraperitoneally (i.p.) (Dunn et al., 1991; 1994). In fact, i.p. administration of IL-1 chronically infused (o, 5, 2, 4 micrograms/24h for 1 week) significantly increased ACTH and corticosterone concentrations in plasma (Sweep et al., 1992).

These results suggest a strong impact of IL-1 on HPA axis. 
Along with the activation of the HPA axis, IL-1 seems to produce changes in the central neurotransmission. IL-1beta, administered i.p. enhances norepinephrine release in the paraventricular nucleus (PVN) (Mohankumar et al., 1993; 1998) and hypothalamus (Dunn, 1988), similar effects have been noticed when IL-1beta was injected i.c.v. These findings suggest a dysregulation of the noradrenergic system by IL-1 administration (Wichers et al., 2002).

Together with the activation of the noradrenergic system, the serotonergic neurotransmission seems to be affected by IL-1 production, for example: IL-1 i.p. injection seems to increase the metabolism of tryptophan and therefore to enhance the production of serotonin and the concentrations of its catabolite the 5-hydroxyindoleacetic acid (5-HIAA) suggesting an increased serotonin turnover and therefore an impairment in the central neurotransmission (Maes, 2001). A study by Zubareva (Zubareva et al., 2001) investigated the role of the serotonergic system, during IL-1 administration, using the pretreatment of p-chlorphenylanine, an inhibitor of serotonin synthesis. This drug blocked the IL-1beta induced decrease in food intake and loss of body weight, but failed to alter the temperature increase and the decrease in communicative activity in rats. This result may indicate a multifactor etiology where $5-\mathrm{HT}$ doesn't act the main role.

Also direct interactions between cytokines and brain regions have been studied: in fact, IL10 injection increases $5-\mathrm{HT}$ concentrations in the $\mathrm{CA}_{1}$ area of the hippocampus of laboratory animals (Broderick, 2002) while IL-1beta inhibits neuronal activity and neuronal plasticity at the hippocampal CA1 synapses (Ikegaya et al., 2003). The inhibitory activity of IL-1beta is known also for the dendrites in raphe nuclei of guinea pigs (Manfridi et al., 2001).

In conclusion IL-1 interacts with several neurotransmitters, e.g. 5-HT, NE at different neuroanatomical levels hypothalamus, hippocampus with an impact on several systems, such as HPA axis.

Depression is accompanied by an enhanced production of pro-inflammatory cytokines including interleukin-6 (IL-6), but also stress or negative stimuli could cause a dysregulation in the pro-inflammatory cytokines and specifically of IL-6. Its overproduction in stressful conditions is partially determinated by the activation of the beta-adrenergic receptors normally elicitated in such conditions.

IL- 6 overproduction together with IL-1 may activate the HPA axis in releasing corticotropinreleasing factor (CRF) and cortisol by increasing prostaglandin E2 production (Song, 2000). IL1 and IL- 6 share common tasks and similar effects both in the periphery and in the central nervous system with alterations in the neurotransmission including 5-HT and dopamine. Even though certain studies don't show any changes in the 5-HT system after IL-6 i.p. injection, others report an increase of a 5-HT catabolite, 5-hydroxyindolacetic acid (5-HIAA) (Zalcman et al., 1994), although with an extent less prominent than that noticed after IL-1 administration. IL-6 and IL-1 may have synergistic effects during mild stress (air puff) in animal models (Song, 2000).

IL-6 seems to exert a more relevant influence on dopamine levels. IL- 6 i.p. injection provokes a decline in the extracellular concentrations of dopamine, after 30 minutes, with an enduring effect up to 2.5 hours (Song, 2000). The effects produced by IL-6 on cerebral neurotransmission do not seem to follow a bi-directional path, at least in healthy individuals. In fact acute depletion of tryptophan or tyrosine (with the subsequent decrease in the $5-\mathrm{HT}$ or cate- 
cholamines levels) does not seem to affect IL- 6 production or to impact the ratings of lowered mood (Harrison et al., 2002).

Stastny et al. (2003) carried out a similar experiment in a population of depressed patients affected by seasonal affective disorder. They found that, even though, plasma levels of IL- 6 and cytokine receptors such as TNF-R1, TNF-R2 were not significantly altered by monoamine depletion, an increased production of neopterin and lowered values of IL-4 soluble receptor (sIL-4r) were noticed. These changes had a time course manner and were associated to an increase in ratings of depressive symptomatology. These results seem to indicate that elevations in neopterin levels and decreases in plasma tryptophan levels have a parallel time course together with significant lower levels of sIL-4r. In other terms it seems that tryptophan depletion along with an impairment of brain $5-\mathrm{HT}$ is related to an activation of a cell-mediated immunity (increased neopterin) and an exacerbation of depressive symptoms accompanied with lowered values of an anti-inflammatory cytokine (sIL-4r).

IL-6 has also a central role during inflammation. This cytokine plays a pivotal role in promoting the production of the C-reactive protein (CRP), which constitutes an important risk factor for myocardial infarction. Individuals with high levels of both IL- 6 and CRP are found to have a 2.6 times probability to die over a 4.6 year period than those who had low levels of both. IL-6 and CRP may play a role in different diseases associated with disability among older adults such as Alzheimer disease or simple aging (Bonaccorso et al., 1998). Elevated levels of IL- 6 may slow muscle repair and accelerate muscle wasting after an injury. According to this theory the presence of IL-6 might be associated with self-rated health (Kiecolt-Glaser, 2002).

These findings show a tight relation between IL-6, HPA activation, dopaminergic and noradrenergic systems.

Interleukin-2 (IL-2) is mainly produced by T-lymphocytes (CD4+, CD8+) and B-lymphocytes. Its administration to humans gives one of the most strong and direct evidence of the potent neuropsychiatric side effects of immunotherapy. Interleukin-2 has been used widely and for a long time to treat patients affected by metastatic cancer. Reports about its side effects are dated mid 8o's and they mostly showed loss of energy, malaise, and fatigue and decreased food intake, only few were designed to carry out the neuropsychiatric effects of IL-2. Denicoff (1987) studied 44 patients with metastatic cancer treated with IL-2 alone or in combination with activated killer cells and found that 15 of the 44 showed severe behavioral changes such as severe agitation that necessitated treatment and physical restraints or emergency psychiatric consultation. Another study by Buter (1993) reported depression after high dose of IL-2. Fourteen of 61 patients experienced neuropsychiatric symptoms. More recently Konsman et al. (2002) found that the $30-45 \%$ of individuals affected by cancer or viral hepatitis $C$ develop depressed mood, anhedonia anxiety and cognitive impairment when administered with interleukin 2 or interferon-alpha.

Capuron et al. (2002) found that administration of IL-2 to cancer patients or to patients affected by viral diseases leads to the development of depressive symptoms and to an increase in MADRS scores and a decrease in tryptophan serum concentration. Tyrosine, another essential amino acid for catecholamines metabolism, did not change significantly during IL-2 treatment while tryptophan concentration significantly decreased. These lowered values 
were associated to the development of suicidal ideation, anorexia, pessimistic thoughts and loss of concentration (Capuron et al., 2002). The depressive symptoms are accompanied by cognitive disturbances and neurovegetative symptoms such as loss of appetite, fatigue or altered sleep. More specifically depressive symptoms and fronto-subcortical-like cognitive alterations develop early (first week of treatment) when patients are given interleukin-2; and later, after 4-8 weeks of treatment, when administered with interferon-alpha (Capuron et al., 2003).

The causal role of interleukin- 2 in the pathophysiology of depression might be explained by the activation of IDO. IL-2 together with IL-1, interferon-gamma (IFN-gamma), and interferon-alpha (IFN-alpha) induces the activation of indoleamine 2, 3-dioxygenase (IDO). IDO is the major tryptophan-catabolizing enzyme in the brain, kidney, lung, spleen, and duodenum, immune cells and induces the catabolism of tryptophan to kynurenine. Over stimulation of IDO determines tryptophan depletion in plasma and therefore reduces the synthesis of $5-\mathrm{HT}$ in the brain (Bonaccorso et al., 2000).

The findings reported above seem to give a positive confirmation about a causal role of cytokines in the pathophysiology of major depression. Tight and complex are the interactions between the alterations in the HPA axis, the dysfunctions in the serotonin neurotransmission, brain and behavior.

The behavioral effects of cytokines were studied initially with IL-1 which was shown to induce, in experimental animals, a specific behavioral complex, called "sickness behavior" characterized by locomotor retardation (Lacosta et al., 1998), hyperalgesia (Maier et al., 1993; 1995), anorexia, soporific effects, and inhibition of sexual behavior in female rats (Anisman et al., 1998). Several authors (Anisman et al., 1999; Dantzer 2001; Konsman et al., 2002) showed the adaptive role of the "sickness behavior" not only as a reflection of a malaise but also as the result of "a centrally-mediated motivational state". Let's take anhedonia as one of the most representative symptom of depression in humans. If we consider anhedonia as a "diminution in perceived reward or pleasure from otherwise rewarding stimuli or responses" we have to take in account the considerably reduced consumption of palatable substances in animals injected by IL-1beta or the bacterial endotoxin, lipopolysaccharide (LPS). This constitutes an attempt to give a valid translation in animal experimental models of what is the most common sign and symptom in depression. This kind of paradigm does not completely succeed in the aim of dissociating the anorexic and anhedonic effect of IL-1beta injection. Merali et al. (2003) tried to improve this model by using a progressive ratio schedule, according to which animals need to progressively increase the amount of work needed in order to obtain a fixed reward. This paradigm may be more accurate in describing the potential anhedonic effect of IL-1 beta.

Peripheral and central injections of recombinant rat IL-1 have been shown to decrease social exploration in rats (Bluthe et al., 1996) and this has been put in relation to a marked increase in extracellular corticosterone levels in the hippocampus (Linthorst et al., 1995, 1997). In rats, acute and chronic administration of IL-1beta significantly increase the latency of escape to a foot shock at the shuttle box (a model for anxiety- and depression-like behavior in animals), whereas repeated IL-1beta administration does not induce a sensitization of these 
behavioral responses (Bonaccorso et al., 2003).

The sickness behavior is not the only effect produced by IL-1 injection: weight loss, fever and depression in social behavior constitute others signs. In fact IL-1 is not the only interleukin which may induce behavioral changes. Similar effects are obtained by administration of IL-2 to animals (Anisman et al., 1998). Also, it's interesting to know that infecting healthy mice $\mathrm{MRL}+/+, \mathrm{C}_{3} \mathrm{H}$.SW and Balb/C with adenovirus vector carrying CDNA for murine IL-6 determined increased sustained serum IL- 6 levels over 5 days, and a rapid decline in preference for sucrose, suggesting that sustained IL- 6 production is an early mechanism in the behavioral and motivation/emotional alterations during chronic inflammatory conditions (Sakic et al., 1997). In humans, a low dose of IL- 6 (0, 5 microgram/Kg body wt) is associated with increased ratings of fatigue, inactivity and difficulties concentrating (Spath-Schwalbe et al., 1998). Also interferons exert behavioral effects in animals. In mice, human interferon-alpha injected i.v. increased the immobility in the forced swimming test, a test regarded as an animal model of depression and the increased in the immobility thought to indicate "behavioral despair" (Bonaccorso et al, 2000).

We have to underline the fact that all the alterations mentioned above are not specific of major depression if taken singularly. For example, IRS activation as described above is not a specific marker for major depression. In fact, it can be found in others disorders like schizophrenia, bipolar disorders, mania, psychological stress, somatization disorders, anxiety and post-traumatic stress disorder (PTSD) (Maes personal communication, 2003). HPA axis is activated also during stress and not only during mood disturbances and the same can be said for serotonin neurotransmission.

Plus, it cannot be traced a unidirectional relation between specific cytokines and a specific behavioral complex or symptom. In fact several cytokines contribute to the same manifestations. Anyhow the combined non-specific dysregulation in IRS, HPA axis, and serotonin system might indicate a specific pattern for major depression disorder.

Examples of this variability can be found in the second and third chapter where it's shown, for fibromyalgia patients, the absence of the activation of the IRS but a dysregulation of the serotonin metabolism with lowered tryptophan concentration; while, for Alzheimer's patients, the results show no significant alterations in the availability of tryptophan to the brain but signs of activation of IRS. The combined, non-specific but peculiar pattern of a dysregulation in IRS, HPA and serotonin system is illustrated in the fourth, fifth and sixth chapter, where are reported the most recent data shown in the international literature on the effects of immunotherapy with interferon-alpha in experimental animals and humans, the alterations of the serotonergic metabolism after repeated administration of interferon-alpha in patients affected by hepatitis $C$ and the subsequent modifications of the cytokine network. The hypothesis pertaining the correlation between these two latter systems and the depressive symptomatology shown by these patients is fully explained in the sixth chapter while the characteristic psychopathology reported by these people is described in the last chapter where it is pointed out the difference with the classic core of symptoms of depression as normally known.

The seventh and the eight chapters give additional informations about the detailed picture describing the links between depression, cytokine network and neurotransmitter alterations. These two chapters show the role of two enzymes DPP-IV and PEP which modulate the activ- 
ity of some cytokines and neuroactive peptides and which are related to the depressive symptomatohology shown by patients affected by hepatitis $C$ and treated with IFN-alpha in an inverse correlation. Lowered activity of these enzymes at the baseline can predict higher depressive and anxiety ratings during IFN-alpha based immunotherapy.

The first chapter shows the difference between acute, repeated and chronic administration of cytokines (IL-beta) on experimental animals on depression behavior resulting that only the acute and chronic administration increase the latency of escape to a foot shock in the shuttle box escape model. 


\title{
Chapter 1
}

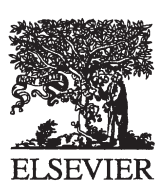

Research report

\section{Behavioral changes in rats after acute, chronic and repeated administration of Interleukin-1beta: relevance for affective disorders}

\author{
Stefania Bonaccorso ${ }^{\text {a,b,c,d }}$, Steven F. Maier ${ }^{\mathrm{b}}$, Herbert Y. Meltzer $^{\mathrm{d}}$, Michael Maes $^{\mathrm{c}, \mathrm{d}, \mathrm{e}, *}$ \\ ${ }^{a}$ Psychiatric Hospital, University 'La Sapienza', Rome, Italy \\ ${ }^{\mathrm{b}}$ University of Colorado at Boulder, Department of Social Psychology, Boulder, CO, USA \\ ${ }^{\mathrm{C}}$ Clinical Research Center for Mental Health, (CRC-MH), Limburg, Belgium \\ 'Department of Psychiatry, Vanderbilt University, Nashville, TN, USA \\ ${ }^{\mathrm{e}}$ Department of Psychiatry, University Hospital of Maastricht, Maastricht, The Netherlands \\ Received 3 April 2001; received in revised form 3 April 2002; accepted 15 April 2002
}

\begin{abstract}
Background: Acute, peripheral and central administration of recombinant rat interleukin-1 $\beta$ (IL-1 $\beta$ ) has been shown to decrease social exploration and locomotor activity and to induce alterations in brain biogenic amines in rats. The aims of this study were to examine whether acute, repeated and chronic administration of IL-1 $\beta$ to rats may interfere with shuttle box escape learning, a model for anxiety- and depression-like behavior. Methods: Sixty-four adult male viral-free SpragueDawley rats (200-300 g weight) housed in groups of four at $25^{\circ} \mathrm{C}$ with a $12: 12$ light:dark cycle were used in the experiments. They were divided into 8 groups, i.e. 4 control and 4 experimental. The latter were divided into an acute group receiving a single intra-peritoneally (I.P.) challenge of $I L-1 \beta$ (tested at the shuttle box 1 and $24 \mathrm{~h}$ later); a chronic group with daily I.P. injections of $\mathrm{IL}-1 \beta$ for 7 days (tested at the shuttle box $1 \mathrm{~h}$ later); and a group with repeated administration, i.e. one I.P. injection on the first day and a second challenge on the seventh day (tested at the shuttle box $1 \mathrm{~h}$ later). The control animals followed the same injecting and testing schedule but were treated I.P. with saline. Results: The acute group treated with one $\Pi L-1 \beta$ challenge and tested $1(P=0.001)$ and $24 \mathrm{~h}(P=0.002)$ later showed significant time elongations in the escape trials. The animals treated chronically with IL- $1 \beta$ for seven consecutive days showed a significant increase in the latency at the escape trials $(P=0.0001)$. Repeated administration of $\Pi L-1 \beta$ on the first day and a second on the seventh day did not significantly alter the time elongation in the escape trial. Discussion: Acute and chronic administration of IL- $1 \beta$ significantly increase the latency of escape to a foot shock, whereas repeated IL-1 $\beta$ administration does not induce a sensitization of these behavioral responses.

(C) 2002 Published by Elsevier B.V.
\end{abstract}

Keywords: Interleukin-1 $\beta$ (IL-1 $\beta)$; Depression; Anxiety; Cytokines; Immunology

${ }^{*}$ Corresponding author. Department of Psychiatry and Neuropsychology, University Hospital of Maastricht, Provisorium, Suite 13, Postbus 5800, AZ 6202 Maastricht, The Netherlands. Tel.: +31-43-387-1025; fax: +31-43-387-1026.

E-mail address: http://www.marquiswhoswho.net/MMAES/ (M. Maes), crc-mh@online.be (M. Maes).

0165-0327/\$ - see front matter (c) 2002 Published by Elsevier B.V. doi: 10.1016/S0165-0327(02)00118-0

\section{Introduction}

There are now many studies showing that an activation of the inflammatory response system 
(IRS) may accompany major depression (Maes et al., 1992; Muller et al., 1993; Seidel et al., 1995; Sluzewska et al., 1996a,b; Connor and Leonard, 1998). $\mathrm{IL}-1 \beta$ is one of the pro-inflammatory cytokines which is considered to play a role in the underlying phenomena of mood disorders (Maes et al., 1993; Maes, 1995). An increased production of IL-1 $\beta$ and increased serum concentrations of the IL-1 receptor antagonist (IL-1RA) have been found in depression (Maes et al.,1995, 1997; Song et al., 1998; Anisman et al., 1999a,b). The increased production of IL-1 $\beta$ could also provoke some of the hormonal abnormalities observed in depression, since it is known that an increased production of IL-1 $\beta$ stimulates the HPAaxis (Maes et al., 1993; Anforth et al., 1998). In experimental animals, IL-1 $\beta$ may also induce a specific behavioral complex, called sickness behavior, characterized by locomotor retardation, sleep disorders, soporific effects, anorexia, weight loss, hyperalgesia, decreased social exploration, and inhibition of sexual behavior in female rats (Lacosta et al., 1998; Maier et al., 1993; Anisman et al., 1998; Bluthe et al., 1996). It is now well established that an inflammatory challenge as evoked by bacterial endotoxin or $\mathrm{IL}-1$ administration induces autonomic, endocrine, and behavioral responses that are controlled by the brain (Linthorst and Reul, 1998). Peripheral injection of LPS causes sickness behavior and a dramatic increase in preoptic extracellular concentrations of noradrenaline and a marked increase in hippocampal extracellular serotonin levels, responses which are mediated through IL-1 and prostaglandins (Linthorst and Reul, 1998). Thus, peripheral IRS activation results in highly differentiated changes in brain neurotransmission, probably subserving the coordinate processing of immune information in circuits involved in autonomic, neuroendocrine, and behavioral regulation (Linthorst and Reul, 1998). The central effects of peripheral immune activation have probably adaptive significance and relevance to sickness behavior; however, pathological outcomes, such as depression and anxious-like states, could develop should proinflammatory cytokines, such as IL- $1 \beta$, be overproduced or dysregulated (Maes, 1995; Zalcman et al., 1998). There is also evidence that IL-1 $\beta$ impairs long term potentiation (LTP) in the hippocampus, which has been proposed as a biological substrate for learning and/or memory (Lynch, 1998).
Repeated administration of IL-1 $\beta$ may induce long-term effects (sensitization) in, for example, stress hormones. For example, exposure of IL-1 $\beta$ primed rats 1-2 weeks later to footshocks results in exaggerated cortisol responses as compared to vehicle-primed controls, suggesting that IL-1 induces long-lasting hyperresponsiveness to stressors (Tilders and Schmidt, 1998). Eleven days after IL-1 $\beta$ administration, a second IL-1 $\beta$ challenge causes a marked depletion of the vasopressin stores in the external zone of the median eminence within $2 \mathrm{~h}$, which is not seen in rats treated with vehicle 11 days earlier (Schmidt et al., 1995). However, little is known about the delayed or long lasting behavioral effects of $I L-1 \beta$ administration.

The aims of the present study are to examine the behavioral changes in rats after acute, repeated, and chronic injections of IL-1 $\beta$.

\section{Subjects and methods}

Adult male viral-free Sprague-Dawley rats 200 $300 \mathrm{~g}$ weight housed in groups of four at $25^{\circ} \mathrm{C}$ with a 12:12 light:dark cycle were used in all experiments. Food and water were freely available. There were 8 rats in each group. Care and use of the animals were in accordance with protocols approved by the University of Colorado Institutional Animal Care and Use Committee. Recombinant human IL-1 $\beta$ (rhIL-1 $\beta$ ) was a gift by the National Cancer Institute, Division of Cancer Treatment (NCI). The drug was diluted in sterile saline immediately prior to the injection intraperitoneally (I.P.) at a dose of $5 \mu \mathrm{g} / \mathrm{kg}$. Control animals were injected with an equal volume of saline. Animals were divided in 8 groups of 8 rats each (4 experimental and 4 controls). The acute group received a single I.P. challenge of IL-1 $\beta$ and was tested at the shuttle box 1 and $24 \mathrm{~h}$ later. The chronic group had been given daily I.P. injections of IL-1 $\beta$ for 7 days and tested at the shuttle box $1 \mathrm{~h}$ later. Control animals followed the same injecting and testing schedule but were treated I.P. with saline. The repeated groups received one I.P. injection with IL-1 $\beta$ or saline on the first day and a second challenge with I.P. IL-1 $\beta$ on the seventh day; the rats were tested at the shuttle box $1 \mathrm{~h}$ later.

The shuttle box testing started 1 or $24 \mathrm{~h}$ later than the last injection with a 10 -min behavioral observation by 
an experimenter unaware of group membership. This 10-min observation period was followed by two (fixed ratio) FR-1 escape trials. Shock intensity was $0.8 \mathrm{~mA}$ and the shocks occurred with an average interictal interval of $60 \mathrm{~s}$. Shocks terminate automatically after $30 \mathrm{~s}$ if escape had not occurred and, accordingly, a $30 \mathrm{~s}$ latency is assigned to those animals. The two FR-1 escape trials were followed by a 20 -min observation period, conducted as before. Three more FR-1 escape trials and then 25 FR-2 escape trials followed the 20min post-FR-1 observation period.

\subsection{Statistics}

The data were analyzed by means of analyses of variance (ANOVAs) with the treatment condition (IL$1 \beta$ versus placebo) and the six time points as factors. All tests were two-tailed $(P=0.05)$.

\section{Results}

Fig. 1 shows the measurements in the different test conditions. The acute group, treated with one administration of IL- $1 \beta$ and tested $1 \mathrm{~h}$ later, showed a significant time elongation in the latency of the escape trials as compared with control rats $(F=11.6$;

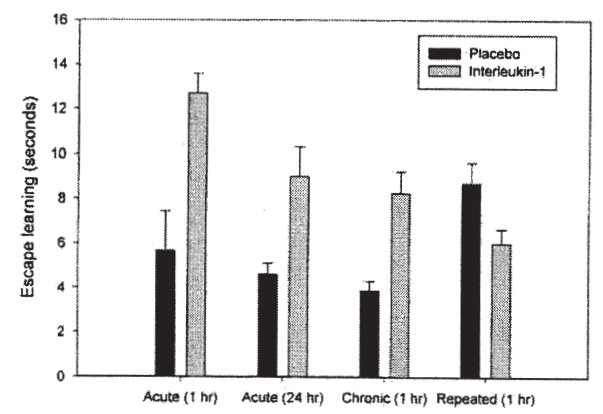

Fig. 1. Effects of Interleukin-1 $\beta$ versus saline in four groups of rats, i.e. two acute groups treated with one administration of IL-1 $\beta$ and tested 1 and $24 \mathrm{~h}$ later at the shuttle box; one chronic group treated with seven challenges of IL-1 $\beta$ for seven consecutive days and tested $1 \mathrm{~h}$ later at the shuttle box; and one group of rats treated with two repeated injections of IL- $1 \beta$ on the first and on the seventh day and tested at the shuttle box $1 \mathrm{~h}$ later. $d f=1.84 ; P=0.001)$. The acute group treated with one injection of $\mathrm{IL}-1 \beta$ but tested at the shuttle box $24 \mathrm{~h}$ later showed a significant increase in latency of the escape trials $(F=10.4 ; d f=1.84 ; P=0.002)$. The chronic group, treated with seven challenges of IL-1 $\beta$ for seven consecutive days, showed a significant increase in the latency at the escape trials $(F=20.2 ; d f=1.84 ; P=0.0001)$. Rats which were treated with two repeated injections of $I L-1 \beta$ on the first and on the seventh day and tested at the shuttle box $1 \mathrm{~h}$ later did not have a significantly different behavioral response as compared with placebo injected rats.

\section{Discussion}

Our results show a significant increase in the latency of escape to a foot shock in experimental animals treated acutely with IL-1 $\beta$. Acute bilateral infusion into the hippocampus of lipopolysaccharide (LPS), which increases the production of IL-1 $\beta$, blocks the escape learning (Ma and Zhu, 1997a).

Acute intrahippocampal infusion of IL-6, another proinflammatory cytokine produced by, amongst other things, cells of the monocyte-macrophage lineage, impairs learning acquisition of active avoidance by enhancing the latency of avoidance in rats tested at the one-way shuttle box (Ma and Zhu, 1997b).

A major finding of this study is that animals treated chronically with $\mathrm{IL}-1 \beta$ for seven consecutive days showed a highly significant increase in the latency at the escape trials. The persistent increase in the escape latency found after 7 days of $\mathrm{IL}-1$ administration shows that there is no habituation in this response. These findings suggest that chronic inflammatory states characterized by increased $\mathrm{IL}-1$ production may be accompanied by a defect in avoidance learning. I.P. administration of chronically infused IL-1 for 1 week significantly elicited ACTH and corticosterone concentrations in plasma (Sweep et al., 1992). Transgenic mice, which express IL-6 chronically from astrocytes in the brain, exhibit dose- and age-related deficits in avoidance learning that closely corresponded with specific progressive neuropathological changes (Heyser et al., 1997).

In the present study we were unable to find that repeated administration of $I L-1 \beta$ on the first day and 
a second on the seventh day significantly induced time prolongation in the escape trial. These findings suggest that there is no sensitization in the increased latency at the escape trials following $I L-1 \beta$ administration. This contrasts the effects of $\mathrm{IL}-1 \beta$ inducing sensitization in, for example, stress hormones, such as corticosterone and vasopressin (Tilders and Schmidt, 1998; Schmidt et al., 1995).

It has been shown that inescapable shock (IS) may interfere with avoidance escape (Jackson et al., 1980). Thus, it appears that acute and chronic administration of IL-1 $\beta$ exerts effects on avoidance behavior comparable to those obtained by IS although the effects may be less pronounced. Indeed, the average of latency for IS is around $20 \mathrm{~s}$. In rats, exposure to IS raises brain IL-1 $\beta 2 \mathrm{~h}$ after IS but no longer after $24 \mathrm{~h}$ (Nguyen et al., 1998, 2000). IS elevates IL- $1 \beta$ protein in the pituitary both in intact and adrenalectomized rats. Intracerebroventricular (I.C.V.) administration of the IL-1 receptor antagonist (IL-1RA) before IS blocks the subsequent interference with escape learning and enhancement of fear conditioning normally produced by this treatment (Maier and Watkins, 1995)

Some data suggest that IL-1 $\beta$ may play a key role in mediating the inhibitory effect of stress on long term potentiation (Vereker et al., 2001). Indeed, there is an inverse relationship between $\mathrm{IL}-1 \beta$ concentrations in the dentate gyrus and LTP in aged rats, stressed rats, and rats pretreated with IL- $1 \beta$. One mechanism is that $\mathrm{IL}-1 \beta$ stimulates the production of reactive oxygen species which triggers lipid peroxidation which in turn causes depletion of membrane arachidonic acid that correlates with impaired LTP (Murray and Lynch, 1998; Vereker et al., 2000). There is behavioral evidence that central IL- $1 \beta$ administration and agents that induce central IL-1beta activity impair the consolidation of memories that depend on the hippocampal formation (Rachal Pugh et al., 2001). Therefore, it could be argued that the failure of the avoidance response after IL- $1 \beta$ administration may reflect an impairment in short term memory rather than a primary change in the anxiety or mood state. Depressed cognitive functioning (disrupted acquisition), however, is another feature of pyrogen-induced sickness behavior (Aubert et al., 1995).

The two-way shuttle box avoidance test has been used as a model for anxiety (Fernandez-Teruel et al., 1991) and mood state (Steimer et al., 1997) in animals. Anxiolytic drugs may improve the early acquisition of avoidance. Treatment with nortriptyline for 4 or 6 days may counteract the escape deficits produced by IS (Telner and Singhal, 1981). $\mathrm{IL}-1 \beta$ is known to stimulate a myriad of illnessrelated outcomes such as sickness behavior, depression-like symptoms and hypothalamic-pituitary-adrenal (HPA) activation (Fleshner et al,, 1995). Activation of peripheral immune cells leads to increases of IL-1 $\beta$ mRNA immunoreactivity, and protein levels in brain and pituitary. Peripherally released $I L-1 \beta$ has potent effects on the central nervous system. One of the primary routes of peripheral cytokine signaling is through stimulation of the peripheral vagus nerve (Bluthe et al., 1996; Maier et al., 1998). Evidence suggests that IL-1 $\beta$ may activate subdiaphragmatic vagal afferents. Experiments in rats demonstrate that subdiaphragmatic vagal transection both attenuates acquisition and facilitates extinction of conditioned taste aversions induced by I.P. administration of IL- $1 \beta$ (Goehler et al., 1995). It has been shown that subdiaphragmatic, but not hepatic vagotomy, blocked the biochemical and hormonal effects of rhIL-1 $\beta$ (Fleshner et al., 1995). I.P. injection of IL$1 \beta$ cannot exert its central actions after vagotomy (Bluthe et al., 1996).

In conclusion, the results of the present study show that acute as well as sub-chronic administration of $\mathrm{IL}-1 \beta$ may significantly interfere with the two-way shuttle box escape. One explanation is that IL-1 $\beta$ may interfere with anxiety or mood state through activation of subdiaphragmatic vagal afferents.

\section{References}

Anforth, H.R., Bluthe, R.M., Bristow, A., Hopkins, S., Lenczowski, M.J., Luheshi, G., Lundkvist, J., Michaud, B., Mistry, Y Van Dam, A.M., Zhen, C., Dantzer, R., Poole, S., Rothwell, N.J., Tilders, F.J., WoIIman, E.E., 1998. Biological activity and brain actions of recombinant rat interleukin-1alpha and interleukin-1 beta. Eur. Cytokine Netw. 9, 279-288.

Anisman, H., Kokkinidis, L., Borowski, T., Merali, Z., 1998. Differential effects of interleukin (IL)-1beta, IL-2 and IL-6 on responding for rewarding lateral hypothalamic stimulation. Brain Res. 779, 177-187. 


\section{S. Bonaccorso et al. / Journal of Affective Disorders 77 (2003) 143-148}

Anisman, H., Ravindran, A.V., Griffiths, J., Merali, Z., 1999a. Interleukin-1 beta production in dysthymia before and after pharmacotherapy. Biol. Psychiatry 46, 1649-1655.

Anisman, H., Ravindran, A.V., Griffiths, J., Merali, Z., 1999b. Endocrine and cytokine correlates of major depression and dysthy mia with typical or atypical features. Mol. Psychiatry 4, 182-188

Aubert, A., Vega, C., Dantzer, R., Goodall, G., 1995. Pyrogens specifically disrupt the acquisition of a task involving cognitive processing in the rat. Brain Behav. Immun. 9, 129-148.

Bluthe, R.M., Michaud, B., Kelley, K.W., Dantzer, R., 1996 Vagotomy attenuates behavioural effects of interleukin-1 injected peripherally but not centrally. Neuroreport 7, 1485-1488.

Connor, T.J., Leonard, B.E., 1998. Depression, stress and immunological activation: the role of cytokines in depressive disorders. Life Sci. 62, 583-606.

Fernandez-Teruel, A., Escorihuela, R.M., Nunez, J.F., Zapata, A., Boix, F., Salazar, W., Tobena, A., 1991. The early acquisition of two-way (shuttle-box) avoidance as an anxiety-mediated behavior: psychopharmacological validation. Brain Res. Bull. $26,173-176$.

Fleshner, M., Goehler, L.E., Hermann, J., Relton, J.K., Maier, S.F. Watkins, L.R., 1995, Interleukin-1 beta induced corticosterone elevation and hypothalamic NE depletion is vagally mediated. Brain Res. Bull. 37, 605-610.

Goehler, L.E., Busch, C.R., Tartaglia, N., Relton, J., Sisk, D Maier, S.F., Watkins, L.R., 1995. Blockade of cytokine induced conditioned taste aversion by subdiaphragmatic vagotomy: further evidence for vagal mediation of immune-brain com munication. Neurosci. Lett. 185, 163-166.

Heyser, C.J., Masliah, E., Samimi, A., Campbell, I.L., Gold, L.H., 1997. Progressive decline in avoidance learning paralleled by inflammatory neurodegeneration in transgenic mice expressing interleukin 6 in the brain. Proc. Natl. Acad. Sci. U.S.A. 94 $1500-1505$.

Jackson, R.L., Alexander, J.H., Maier, S.F., 1980. Learned helplessness, inactivity, and associative deficits: effects of inescapable shock on response choice escape learning. $J$. Exp. Psychol. Anim. Behav. Process. 6, 1-20.

Lacosta, S., Merali, Z., Anisman, H., 1998. Influence of interleukin-1 beta on exploratory behaviors, plasma ACTH, corticosterone, and central biogenic amines in mice. Psychopharmacology (Berl.) 137, 351-361.

Linthorst, A.C., Reul, J.M., 1998. Brain neurotransmission during peripheral inflammation. Ann. N Y Acad. Sci. 840, 139-152.

Lynch, M.A., 1998. Age-related impairment in long-term potentiation in hippocampus: a role for the cytokine, interleukin- 1 beta? Prog. Neurobiol. 56, 571-589.

Ma, T.C., Zhu, X.Z., 1997a. Intrahippocampal infusion of interleukin-6 impairs avoidance learning in rats. Chung Kuo Yao $\mathrm{Li}$ Hsueh Pao. 18, 121-123.

Ma, T.C., Zhu, X.Z., 1997b. Suppression of lipopolysaccharideinduced impairment of active avoidance and interleukin-6-induced increase of prostaglandin E2 release in rats by indomethacin. Arzneim.-Forsch. 47, 595-597.

Maes, M., 1995. Evidence for an immune response in majo depression: a review and hypothesis. Prog. Neuropsychopharmacol. Biol. Psychiatry 19, 11-38.
Maes, M., Lambrechts, J., Bosmans, E., Jacobs, J., Suy, E., Vandervorst, C., de Jonckheere, C., Minner, B., Raus, J., 1992. Evidence for a systemic immune activation during depression: results of leukocyte enumeration by flow cytometry in conjunction with monoclonal antibody staining. Psychol. Med. 22, 45-53.

Maes, M., Bosmans, E., Meltzer, H.Y., Scharpe, S., Suy, E., 1993. Interleukin-1 beta: a putative mediator of HPA axis hyperactivity in major depression? Am. J. Psychiatry 150, 1189-1193.

Maes, M., Vandoolaeghe, E., Ranjan, R., Bosmans, E., Bergmans, R., Desnyder, R., 1995. Increased serum interleukin-1-receptorantagonist concentrations in major depression. J. Affect. Disord. $36,29-36$.

Maes, M., Bosmans, E., De Jongh, R., Kenis, G., Vandoolaeghe, E., Neels, H., 1997. Increased serum IL-6 and IL-1 receptor antagonist concentrations in major depression and treatment resistant depression. Cytokine 9, 853-858.

Maier, S.F., Watkins, L.R., 1995. Intracerebroventricular interleukin-1 receptor antagonist blocks the enhancement of fear conditioning and interference with escape produced by inescapable shock. Brain Res. 695, 279-282.

Maier, S.F., Wiertelak, E.P., Martin, D., Watkins, L.R., 1993 Interleukin-1 mediates the behavioral hyperalgesia produced by lithium chloride and endotoxin. Brain Res. 623, 321-324.

Maier, S.F., Goehler, L.E., Fleshner, M., Watkins, L.R., 1998. The role of the vagus nerve in cytokine-to-brain communication. Ann. N Y Acad. Sci. 840, 289-300.

Muller, N., Hofschuster, E., Ackenheil, M., Mempel, W., Eckstein, R., 1993. Investigations of the cellular immunity during depression and the free interval: evidence for an immune activation in affective psychosis. Prog. Neuropsychopharmacol. Biol. Psychiatry 17, 713-730.

Murray, C.A., Lynch, M.A., 1998. Evidence that increased hippocampal expression of the cytokine interleukin-1 beta is a common trigger for age- and stress-induced impairments in long-term potentiation. J. Neurosci. 18, 2974-2981.

Nguyen, K.T., Deak, T., Owens, S.M., Kohno, T., Fleshner, M. Watkins, L.R., Maier, S.F., 1998. Exposure to acute stress induces brain interleukin-1 beta protein in the rat. J. Neurosci. $18,2239-2246$

Nuygen, T., Deak, T., Will, M.J., Hansen, M.K., Hunsaker, B.N., Fleshner, M., Watkins, L.R., Maier, S.F., 2000. Time course and corticosterone sensitivity of the brain, pituitary, and serum interleukin-1beta protein response to acute stress. Brain Res. 859, 193-201.

Rachal Pugh, C., Fleshner, M., Watkins, L.R., Maier, S.F., Rudy, J.W., 2001. The immune system and memory consolidation: role for the cytokine IL-1 beta. Neurosci. Biobehav. Rev. 25 , 29-41.

Schmidt, E.D., Janszen, A.W., Wouterlood, F.G., Tilders, F.J., 1995. Interleukin-1-induced long-lasting changes in hypothalamic corticotropin-releasing hormone (CRH)- neurons and hyperresponsiveness of the hypothalamus-pituitary-adrenal axis. J. Neurosci. 15, 7417- 7426

Seidel, A., Arolt, V., Hunstiger, M., Rink, L., Behnisch, A., Kirchner, H., 1995. Cytokine production and serum proteins in depression Scand. J. Immunol. 41, 534-538. 
Sluzewska, A., Rybakowski, J.K., Sobieska, M., Wiktorowicz, K., 1996a. Concentration and microheterogeneity glycophorms of alpha-1-acid glycoprotein in major depressive disorder. J. Affect. Disord. 39, 149-155.

Sluzewska, A., Rybakowski, J., Bosmans, E., Sobieska, M., Berghmans, R., Maes, M., Wiktorowicz, K., 1996b. Indicators of immune activation in major depression. Psychiatry Res. 64 $161-167$.

Song, C., Lin, A., Bonaccorso, S., Heide, C., Verkerk, R., Kenis, G., Bosmans, E., Scharpe, S., Whelan, A., Cosyns, P., de Jongh, R., Maes, M., 1998. The inflammatory response system and the availability of plasma tryptophan in patients with primary sleep disorders and major depression. J. Affect. Disord. 49, 211-219.

Steimer, T., la Fleur, S., Schulz, P.E., 1997. Neuroendocrine correlates of emotional reactivity and coping in male rats from the Roman high (RHA/Verh)- and low (RLA/Verh)avoidance lines. Behav. Genet. 27, 503-512.

Sweep, C.G., van der Meer, M.J., Hermus, A.R., Smals, A.G., van der Meer, J.W., Pesman, G.J., Willemsen, S.J., Benraad, T.J., Kloppenborg, P.W., 1992. Chronic stimulation of the pituitary- adrenal axis in rats by interleukin-1 beta infusion: in vivo and in vitro studies. Endocrinology 130, 1153-1164.

Telner, J.I., Singhal, R.L., 1981. Effects of nortriptyline treatment on learned helplessness in the rat. Pharmacol. Biochem. Behav. $14,823-826$.

Tilders, F.J., Schmidt, E.D., 1998. Interleukin-1-induced plasticity of hypothalamic CRH neurons and long-term stress hyperresponsiveness. Ann. N Y Acad. Sci. 840, 65-73.

Vereker, E., O'Donnell, E., Lynch, M.A., 2000. The inhibitory effect of interleukin-1beta on long-term potentiation is coupled with increased activity of stress-activated protein kinases. J. Neurosci. 20, 6811-6819.

Vereker, E., O'Donnell, E., Lynch, A., Kelly, A., Nolan, Y., Lynch, M.A., 2001. Evidence that interleukin-1 beta and reactive oxygen species production play a pivotal role in stressinduced impairment of LTP in the rat dentate gyrus. Eur. J. Neurosci. 14, 1809-1819.

Zalcman, S., Murray, L., Dyck, D.G., Greenberg, A.H., Nance, D.M., 1998. Interleukin-2 and -6 induce behavioral-activating effects in mice. Brain Res. 811, 111-121. 


\title{
Chapter 2
}

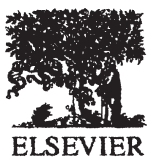

Joumal of Affective Disorders 48 (1998) 75-82

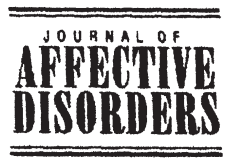

Immune markers in fibromyalgia: comparison with major depressed patients and normal volunteers

Stefania Bonaccorso ${ }^{a, b}$, Ai-hua Lin $^{a, c}$, Robert Verkerk $^{c}$, Fran Van Hunsel ${ }^{a}$, Isabelle Libbrecht ${ }^{a}$, Simon Scharpé ${ }^{c}$, Luc DeClerck ${ }^{d}$, Massimo Biondi ${ }^{b}$, Aleksander Janca ${ }^{e}$, Michael Maes ${ }^{\mathrm{a}, \boldsymbol{f}, *}$

"Clinical Research Center for Mental Health (CRC-MH), Antwerp, Belgium "Department of Psychiatry, University of Rome, La Sapienza, Rome, haly 'Department of Medical Biochemistry, University of Antwerp, Antwerp, Belgium Department of Immunology University of Antwerp, Antwerp, Belgium

'Division of M'ental Health, World Health Organization. Geneva, Switzerland

'Department of Psychiatry, Vanderbilt University, Nashville, TN, USA

Received 9 June 1997; accepted 13 August 1997

\begin{abstract}
Background: There is a high degree of comorbidity between fibromyalgia and major depression. The latter is characterized by signs of immune activation, whereas the immune status in fibromyalgia is not yet elucidated. The aims of the present study were to examine (i) neopterin and biopterin excretion in 24-h urine of patients with fibromyalgia compared with normal volunteers and patients with major depression; and (ii) the effects of subchronic treatment with sertraline (11 weeks) on the urinary excretion of neopterin and biopterin. Methods: Measurements of neopterin, biopterin, pseudouridine, creatinine and uric acid in 24-h urine were performed by means of HPLC in 14 fibromyalgia and ten major depressed patients and 17 normal volunteers. Results: There were no significant differences in urine excretion of the above five analytes between patients with fibromyalgia and normal volunteers. Patients with major depression showed significantly higher urinary neopterin excretion than normal volunteers and fibromyalgia patients. Patients with fibromyalgia and major depression had a significantly increased neopterin/creatinine ratio. Fibromyalgia patients had significantly lower urinary excretion of creatinine than patients with major depression. In fibromyalgia patients, there were no significant effects of sertraline treatment on any of the urine analytes. Conclusions: The findings suggest that fibromyalgia, in contrast to major depression, may not be accompanied ty activation of cell-mediated immunity. Limitation: Other immune markers should be measured in fibromyalgia before drawing definite conclusions. Clinical relevance: Increased urinary excretion of neopterin can be used as a marker for major depression, but not fibromyalgia. (c) 1998 Elsevier Science B.V.
\end{abstract}

Keywords: Fibromyalgia; Neopterin; Cellular immunity; Depression; Selective serotonin reuptake inhibitors; Antidepressants

${ }^{*}$ Corresponding author. Address for correspondence: Clinical Research Center for Mental Health, University Department of Psychiatry, AZ Stuivenberg, Lange Beeldekensstraat 267, 2060 Antwerp, Belgium. Fax: +3234483265 (or +3236332814 )

0165-0327/98/\$19.00 1998 Elsevier Science B.V. All rights reserved.

PII S0165-0327(97)00144-4 


\section{Introduction}

Fibromyalgia is a form of non-articular rheumatism characterized by chronic and widespread musculoskeletal aching, stiffness and pressure hyperalgesia (Yunus et al., 1993). Sleep disturbances, chronic fatigue and depression are commonly associated features (Hernanz et al., 1994). Some studies report a significant magnitude of depressive symptoms in fibromyalgia patients (Hawley and Wolfe, 1993). The latter score higher than normal controls on psychiatric rating scales for c.nxiety and depression (Krag et al., 1994). Hudson et al. (1985) found a higher incidence rate of depression amongst fibromyalgia than rheumatoid arthritis patients. Hawley and Wolfe (1993) found that depressive symptoms may be secondary to fibromyalgia. However, the etiology of fibromyalgia has remained elusive. It is thought that central nervous system mechanisms may be responsible for the majority of the clinical findings in fibromyalgia, i.e. both the musculoskeletal and non-musculoskeletal symptoras, such as depressive symptoms (Clauw, 1995)

Because of the symptomatic similarities between depression and fibromyalgia, measurements of biochemical markers of depression in fibromyalgia are useful (Russell, 1989; Russell et al., 1991). There is now some evidence that major depression is accompanied by activation of cell-mediated immunity and an acute phase response (Joyce et al., 1992; Maes et al., 1992, 1994a, 1995a, 1997; Song et al., 1994; Seidel et al., 1996; Sluzewska it al., 1996). The 'macrophage theory of depression' suggests that an activation of cell-mediated immunity, with increased production of cytokines, such as interleukin-1 (IL-1), interleukin-6 (IL-6) and interferon-gamma (IFNy) may play a role in the pathophysiology of depression (Smith, 1991; Maes, 1995; Maes et al., 1995b). There are few studies which have reported on the immune status in fibromyalgia. No significant differences in lymphocyte subpopulations, could be found between patients with primary fibromyalgia and controls (Hernanz et al., 1994). No statistically significant differences in anti-nuclear-antibody titers were found between fibromyalgia patients and normal controls (Hench and Mitler, 1986; Yunus et al. 1993; Bridges, 1993; Bridges et al., 1996). Others reported increased antibodies titers $(74 \%)$ against serotonin and gangliosides (Klein et al., 1992; Klein and Berg, 1994).

In order to check whether fibromyalgia is accompanied by alterations in cell-mediated immunity we have measured neopterin and biopterin in fibromyalgia patients versus major depressed patients and normal volunteers. There is now some evidence that major depression is accompanied by increased neopterin concentrations and an elevated neopterin/biopterin ratio (Duch et al., 1983; Anderson et al., 1992; Dunbar et al., 1992; Maes et al., 1994b). Neopterin is a low molecular weight not-conjugated pteridin present in the primate's reticulo-endothelial system and produced by activated macrophages after IFN $\gamma$ stimulation. Biopterin is the final catabolic product in the tetrahydrobiopterin pathway. Chemically more stable than tetrahydrobiopterin, biopterin is a useful indicator of the synthesis of tetrahydrobiopterin, which is an essential co-factor in the synthesis of the monoamine precursors, such as DOPA (precursor of dopamine, norepinephrine, epinephrine) and 5-hydroxytryptophan (precursor of serotonin) (Nichol et al., 1985). There are reports that antidepressant drugs, such as tricyclic antidepressants and selective serotonin reuptake inhibitors (SSRIs) show a clinical efficacy in the treatment of fibromyalgia, e.g. improvement of pressure hyperalgesia, pain symptoms, sleep disturbances and depressive symptomatology (Saccomani et al., 1993; Goldenberg, 1989).

The aims of the present study were to examine: (i) the excretion of neopterin and biopterin in patients with fibromyalgia versus normal controls and patients with major depression; and (ii) to examine the effects of treatment with an SSRI, i.e. sertraline, on neopterin and biopterin excretion in fibromyalgia patients.

\section{Materials and methods}

\subsection{Subjects}

Forty-one subjects participated in these studies, i.e. 17 normal volunteers, 14 fibromyalgia patients and ten depressed patients. The study protocol was approved by the Ethical Committee of the University Hospital of Antwerp, Belgium. The fibromyalgia outpatients were selected at the Department of 
Rheumatology, University Hospital of Antwerp, Belgium. The patients fulfilled all criteria for a fibromyalgia diagnosis according to the American College of Rheumatology (ACR) criteria (Wolfe et al., 1990): (1) a history of widespread pain for at least 3 months, i.e. pain in the left side of the body, pain in the right side of the body, pain above and below the waist, axial skeletal pain (cervical spine or anterior chest or thoracic spine or low back pain); and (2) presence of at least 11 tender point sites (measurements performed using digital palpation with a force of $4 \mathrm{~kg}$ ): occiput $\mathrm{L}$ or $\mathrm{R}$, low cervical L or $R$, trapezius $L$ or $R$, supraspinatus $L$ or $R$, second rib $L$ or $R$, lateral epicondyle $L$ or $R$, gluteal $L$ or $R$, and knee $L$ or $R$. For a tender point to be considered 'positive' the subject must state that the palpation was painful. Exclusionary criteria for fibromyalgia patients were the following: presence of major clinical conditions other than fibromyalgia; presence of a major depressive disorder, according to DSM-IV criteria (APA, 1994); suicidal thoughts or a serious risk of suicide; patients who had received fluoxetine within 6 weeks, MAO inhibitors within 2 weeks, other psycho-active drugs within 1 week and antiinflammatory drugs within 4 weeks before baseline use of any psycho-active drugs, except lormetazepam during the study; use of any other medication for the symptorns of fibromyalgia (except paracetamol); or any other medications, physiotherapy or any othe adjuvant therapy (psychotherafy, homeopathy, acupuncture). Most fibromyalgia patients were free of psychotropic drugs prior to the 1-week wash-out period. Prior to this wash-out period, some patients had been taking antidepressants $(n=4)$. The antidepressants used by these patients prior to the 1-week wash-out period were: amitriptyline $(25-75 \mathrm{mg} /$ day, $n=3$ ) or trazodone ( $100 \mathrm{mg} /$ day). After inclusion of the patients, these antidepressants were discontinued and, subsequently, these four patents underwent wash-out period of 1 week. During the study, five patients were treated with lormetazepam in case of severe sleep disorders and three patients were treated with paracetamol on a regular basis.

Ten major depressed inpatient; admitted to the University Department of Psychiatry, Antwerp, Belgium, participated in this study. These patients wer free of any psychotropic drugs for at least 1 week. Patients were classified as having major depression according to the DSM-IV (American Psychiatric Association, 1994) diagnostic criteria. Exclusion criteria were other axis-I diagnoses besides major depression, such as organic mental disorders, schizophrenic and paranoid disorders, primary anxiety disorders, somatoform disorders, psychoactive substance use disorders within the past 6 months, anxiety and eating disorders. Exclusionary criteria for fibromyalgia and depressed patients were: immunocompromised patients, patients suffering from a neurological, inflammatory, endocrine or clinically significant chronic disease, patients receiving drugs with known or potential interaction with immune functions and anticoagulant therapy. Also excluded were patients who were treated with psycho-active medication, except low-dosage benzodiazepines.

Seventeen normal volunteers participated in this study. All subjects had a negative past, present or family history for psychiatric disorders. None was a regular drinker and none had ever been taking psychotropic drugs. All were free of any medications for at least 1 month. All fibromyalgia, depressed and normal subjects had normal blood tests, such as serum electrolytes, urea, liver function tests, such as $\gamma$-GT, SGPT, SGOT, and thyroid function tests, such as FT4 and basal thyroid secreting hormone. Al subjects were free of medical illnesses, such as endocrine, metabolic, or autoimmune disorders (lupus erythematosus, inflammatory bowel disease rheumatoid arthritis, and diabetes), and free of any infections, inflammatory or allergic reactions for at least 2 weeks prior to the blood sampling and free of drugs known to affect immune or endocrine functions and of hormonal preparations.

\subsection{Methods}

The 24-h urine collection started at $11 \mathrm{pm}$. Subjects were instructed to rest during the urine collec tions. Urine was kept at $-20^{\circ} \mathrm{C}$ in dark bottles to prevent the oxidation of labile compounds, such as neopterin and biopterin. The urine collection was considered complete if the creatinine excretion exceeded $0.7 \mathrm{~g} /$ day and urine volume $0.7 \mathrm{l} /$ day. The measurement of pseudouridine (marker of T-RNA turn-over and the metabolic/catabolic state), creatinine and uric acid in urine was performed by means of HPLC (Van Acker et al., 1993). A fluores- 
cence detector was installed to allow quantification of neopterin and biopterin (Motyl et al., 1993). Tender points were evaluated by means of dolorimetry: a trained rheumatologist advanced the instrument at a rate of approximately $1 \mathrm{~kg}$ per second and the patient was instructed to tell when this procedure became painful. The pressure was measured at each of the above 18 tender points. The total fibromyalgic score (right + left) was used in the statistical analyses. A visual analogue scale (VAS) was employed to rate the following items: global fibromyalgic symptoms, stiffness, pain, anergy and sleep disturbances. The 17-iterı Hamilton Depression Rating Scale was completed to measure severity of depression (HDRS; Hamilton. 1960).

Thirteen fibromyalgia patients had repeated 24-h urine collections and measurements of the above clinical ratings both at baseline and after repeated administration of sertraline $(n=7)$ or placebo $(n=$ 6 ) for 11 weeks. The fibromyalgia patients took part in a double-blind, placebo-controlled, multicenter study on the clinical efficacy of sertraline in fibromyalgia (Pfizer, Belgium). Patients were randomized to receive sertraline, $50-100 \mathrm{mg} /$ daily, or placebo for 11 weeks.

\subsection{Statistics}

Group mean differences were checked by means of analysis of variance (ANOVA) or analysis of covariance (ANCOVA). Differences between preand posttreatment measurements were assessed by means of repeated measure design ANOVAs or ANCOVAs. Post-hoc differences among group means were ascertained with Fisher's least significant difference (LSD). Relationships between variables were assessed with Pearson's product-moment or the point-biserial correlation coefficients and through multiple regression analysis. The independence between classification systems was checked with the analysis of contingence $\left(x^{2}\right.$ test).

\section{Results}

There were no significant differences $(F=2.4$, df $=2 / 38, P=0.1$ ) in age between normal controls (mean $=41.8 \pm 14.0$ years), fibromyalgia patients (mean $=51.0 \pm 6.9$ years) and depressed patients (mean $=45.3 \pm 12.4$ years). There were no significant differences $\left(\chi^{2}=0.7, \mathrm{df}=2, P=0.7\right)$ in the male/ female ratio between normal controls $(11 / 6)$, fibromyalgia $(11 / 3)$ and depressed patients $(7 / 3)$. There were no significant differences in mean urinary volume $(F=0.2, \mathrm{df}=2 / 38, P=0.85)$ between normal volunteers $($ mean $=1.80 \pm 0.841)$, fibromyalgia (mean $=2.00 \pm 0.97 \mathrm{l}$ ) and depressed patients (mean $=1.84 \pm 1.181$ )

Table 1 shows the correlation matrix between the biological analytes, age and gender. There were significant positive correlations between neopterin and biopterin, neopterin and creatinine, neopterin and pseudouridine, and between biopterin and pseudouridine. No significant correlations were found between age and neopterin, biopterin, creatinine, pseudouridine, and uric acid concentrations. Point-biserial correlations showed that the excretion of neopterin creatinine and uric acid, but not biopterin and

Table 1

Intercorrelation-matrix between 24-h urinary excretion of neopterin, biopterin, creatinine, pseudouridine, uric acid and age/sex

\begin{tabular}{|c|c|c|c|c|c|c|}
\hline Variables & Neopterin & Biopterin & Creatinine & Pseudouridine & Uric acid & Gender \\
\hline Biopterin & $0.82^{* * *}$ & & & & & \\
\hline Creatinine & $0.82 * * *$ & $0.69 * * *$ & & & & \\
\hline Pseudouridine & $0.74^{* * * *}$ & $0.88 * * *$ & $0.51^{* * *}$ & & & \\
\hline Uric acid & $0.63^{* * * *}$ & $0.43 * *$ & $0.68^{* * * *}$ & $0.41 * *$ & & \\
\hline Gender & $0.37 *$ & 0.21 & $0.62^{* * *}$ & 0.11 & $0.44^{* * *}$ & \\
\hline Age & 0.06 & 0.09 & 0.10 & 0.01 & -0.22 & 0.02 \\
\hline
\end{tabular}

All resuls of Pearson's or point biserial (gender: men $=1$, women $=0$ ) correlation analyses, pooled over the three study groups of nom Alluters, fibromyalgia and depressed putients $(n=41)$.

$* P<0.05$.
$* * P<0.01$

$* * * P<0.001$. 
Table 2

Measurements of 24-h urinary excretion of neopterin, biopterin, creatinine, pseudouridine and uric acid in normal volunteers (NV), fibromyalgia (FM) and major depressed (MI)) patients

\begin{tabular}{|c|c|c|c|c|c|c|c|}
\hline Groups & $\begin{array}{l}\text { Neopterin } \\
\mu \mathrm{M}\end{array}$ & $\begin{array}{l}\text { Biopterin } \\
\mu \mathrm{M}\end{array}$ & $\begin{array}{l}\text { Creatinine } \\
\mathrm{mM}\end{array}$ & $\begin{array}{l}\text { Pseudouridine } \\
\mu \mathrm{M}\end{array}$ & $\begin{array}{l}\text { Uric acid } \\
\mathrm{mM}\end{array}$ & $\begin{array}{l}\text { Neopterin/ } \\
\text { biopterin ratio }\end{array}$ & $\begin{array}{l}\text { Neopterin } / \\
\text { creatinine } \times 100\end{array}$ \\
\hline $\mathrm{HV}$ & $2.21(1.50)$ & $1.40(0.80)$ & $7.96(4.00)$ & $175(92)$ & $6.23(4.49)$ & $1.66(0.63)$ & $2.75(1.19)$ \\
\hline FM & $2.10(1.38)$ & $1.70(1.70)$ & $5.37(3.58)$ & $175(167)$ & $4.60(2.89)$ & $1.94(1.32)$ & $4.59(3.54)$ \\
\hline MD & $4.14(3.00)$ & $3.08(3.10)$ & $10.58(6.62)$ & $180(175)$ & $6.02(2,76)$ & $1.96(0.93)$ & $3.90(0.88)$ \\
\hline ANCOVA & & & . & & & & \\
\hline$F$ & 5.7 & 3.1 & 6.1 & 0.00 & 0.3 & 0.2 & 5.4 \\
\hline df & $2 / 35$ & $2 / 32$ & $2 / 35$ & $2 / 34$ & $3 / 35$ & $2 / 32$ & $2 / 35$ \\
\hline$P$ & 0.007 & 0.056 & 0.006 & 0.9 & 0.8 & 0.8 & 0.009 \\
\hline
\end{tabular}

All data are shown as mean ( \pm S.D.).

"All results of ANCOVAs with age, gender and urine volume as covariates.

pseudouridine, were significantly higher in males than in females. In any case, subsequent statistical analyses were adjusted for possible effects of age and sex, by using age and gender as covariates in regression analyses.

Table 2 shows the measurements of neopterin, biopterin, creatinine, pseudouridine, uric acid, neopterin/biopterin and neopterin/creatinine ratio in the three study groups. By means of ANCOVAs (with age, gender and urine volume as covariates) significant differences in neopterin, creatinine and the neopterine/creatinine ratio were found between the three study groups. Urinary neopterin excretion was significantly higher in major depressed patients than in the other two study groups. Creatinine excretion was significantly lower in fibromyalgia than in major depressed patients. The neopterin/creatinine ratio was significantly higher in major depressed and fibromyalgia patients than in normal subjects. There was a trend toward higher urine biopterin concentration in depressed patients than in normal volunteers and fibromyalgia patients.

In fibromyalgia patients, there was a significant and positive correlation between anergy and the neopterin/creatinine ratio $(r=0.76, P=0.002)$. There was a trend toward a negative correlation between anergy and creatinine $(F=4.4, P=0.06$, i.e. significance of regression coefficients obtained after multiple regression with anergy and gender as explanatory variables). There was a trend toward a positive correlation between the neopterin/creatinine ratio and duration of fibromyalgia $(r=0.53, P=$ $0.057)$ and global fibromyalgia symptomatology $(r=$ $0.52, P=0.055$ ).

Table 3 shows the measurements of neopterin, biopterin, creatinine, pseudouridine and uric acid before and after treatment with sertraline, 50-150 $\mathrm{mg} /$ day, or placebo. By means of repeated measure

Table 3

Measurements of 24-h urinary excretion of neopterin, biopterin, creatinine, pseudouridine and unic acid in fbromyalgia patients both at baseline and after treatment (end-point) with sertraline or placebo

\begin{tabular}{lllllll}
\hline Treatment & Condition & $\begin{array}{l}\text { Neopterin } \\
\mu \mathrm{M}\end{array}$ & $\begin{array}{l}\text { Biopterin } \\
\mu \mathrm{M}\end{array}$ & $\begin{array}{l}\text { Creatinine } \\
\mathrm{mM}\end{array}$ & $\begin{array}{l}\text { Pseudouridine } \\
\mu \mathrm{M}\end{array}$ & $\begin{array}{l}\text { Uric Acid } \\
\mathrm{mM}\end{array}$ \\
\hline Placebo & Basal & $1.54(0.67)$ & $1.23(0.82)$ & $4.98(2.76)$ & $119(67)$ & $\begin{array}{l}4.87(1.78) \\
\text { Sertraline }\end{array}$ \\
& End-point & $1.47(0.99)$ & $0.84(0.75)$ & $5.11(3.50)$ & $126(82)$ & $5.26(3.14)$ \\
& Basal & $2.16(1.51)$ & $1.95(1.46)$ & $6.05(4.45)$ & $165(129)$ & $4.87(3.64)$ \\
& End-point & $2.31(2.37)$ & $1.32(0.89)$ & $4.25(4.43)$ & $114(138)$ & $3.43(2.34)$ \\
ANCOVA & F & 0.00 & 0.2 & 0.9 & 0.7 & 1.0 \\
& df & $1 / 10$ & $1 / 7$ & $1 / 11$ & $1 / 8$ & $1 / 11$ \\
& $\mathrm{P}$ & 0.3 & 0.7 & 0.6 & 0.6 & 0.3 \\
\hline
\end{tabular}

All data are presented as mean $( \pm$ S.D.).

-All results of repeated measures design ANOVAs, showing the $F$ values for the interaction time $\times$ type of trearment. 
design ANOVAs, no significant interactions between time $\times$ type of treatment could he found for any of the analytes. There were no significant differences between the baseline $($ mean $=2.01 \pm 1.01 \quad 1)$ and posttreatment $($ mean $=2.08 \pm 1.03$ l) $24-\mathrm{h}$ urine volumes $(F=0.1, \mathrm{df}=1 / 11, P=0.7)$ and no significant time $\times$ type of treatment interaction $(F=1.6$, df $=1 / 11, P=0.2$ ). Repeated measure design ANCOVAs, with 24-h urine volume as covariates, did not change any of the above results. Repeated measure design ANOVA did not show any difference in the total baseline $($ mean $=46.1 \pm 14.6)$ and posttreatment (mean $=47.5 \pm 18.9$ ) myalgic scores $(F=$ 0.3 , $\mathrm{df}=1 / 11, P=0.5$ ); the interaction time $\times$ treatment, i.e. sertraline or placebo, was not significant $(F=0.05, \mathrm{df}=1 / 11, P=0.8)$. These findings suggest that there were no significant effects of sertraline treatment either on the urine analytes or on the clinical state of the patients.

\section{Discussion}

In the present study we found no signs of immune activation in fibromyalgia as assessed by means of neopterin excretion in urine. In addition, we found no significant relationships betweisn neopterin excretion and any of the fibromyalgic symptoms, such as tender points, pain, stiffness, dizpression or sleep disorders, and no significant effects of treatment with sertraline on urinary neopterin excretion. These findings extend those of Hernanz et al. (1994), who could not find significant changes in peripheral blood B, T, CD4 or CD8 lymphocytes. The increase in the neopterin/creatinine ratio found in fibromyalgia patients is probably not immunologically related, because it was attributable to a lower excretion of creatinine. Lower excretion of creatinine in fibromyalgia has also been reported by Jacobsen et al. (1993). This phenomenon was attributed to diminished physical activity in fibromyalgic patients. Jacobsen et al. (1992), (1.993) reported that the voluntary physical capacity for work is lower in fibromyalgia than in normal humans. This finding seems to be supported by the results of the present study that there is a highly significant correlation between anergy and the neopterin/creatinine ratio. There was a trend toward a significant inverse correlation between anergy and urinary creatinine excretion. Lijnen et al. (1985), on the other hand, were unable to find that strenuous exercise induced significant increases in mean creatinine excretion in healthy young men.

In accordance with previous studies (Duch et al., 1983; Anderson et al., 1992; Dunbar et al., 1992; Maes et al., 1994b), we found that the neopterin concentrations were significantly higher in depressed patients than in normal controls. As a marker of immune activation and increased production of proinflammatory cytokines, such as IFNy, the present findings support the 'macrophage theory of depression' (Smith, 1991; Maes, 1995). Thus, it appears that the pathophysiology of fibromyalgia and major depression may be different, i.e. immune activation being specific for the latter. By inference, the comorbidity between both disorders or the presence of depressive symptoms in fibromyalgia patients (Hawley and Wolfe, 1993; Krag et al., 1994; Hudson et al., 1985) may not be related to activation of cell-mediated immunity. However, lower concentrations of plasma tryptophan, the precursor of serotonin (5-HT) were observed in fibromyalgia patients (Russell, 1989; Russell et al., 1991; Yunus et al., 1992). A significant inverse correlation between tryptophan concentrations and morning pain responsivity in those patients may suggest a direct involvement of lower tryptophan availability in the symptomatology of the disease (Hench and Mitler, 1986; Puttini and Caruso, 1992). It is well known that cytokines, such as IFNy, IL-1 and IL-2, may induce indoleamine 2,3-dioxygenase (IDO) (Hu et al., 1995), a key-enzyme which catabolizes tryptophan (Takikawa et al., 1984). Immune activation is accompanied by a decreased availability of tryptophan to the brain due to IDO-induced catabolism of tryptophan (Takikawa et al., 1984; Heyes and Quearry, 1991; Saito et al., 1992). Thus, our findings that there are no significant alterations in neopterin excretion, an index of IFN $\gamma$-induced macrophage activity (Giovannoni et al., 1997), do not exclude the existence of immune activation related to other proinflammatory cytokines, e.g. IL-1 and IL-2.

\section{Acknowledgements}

The research reported was supported in part by the Clinical Research Center for Mental Health (CRC- 
S. Bonaccorso et al. I Journal of Affective Disorders 48 (1998) 75-82

MH), Antwerp, the Fund for Scientific Research, Vlaanderen, Belgium; the Michael Kaplan Investigator Award to Dr.M.Maes (NARSAD).

\section{References}

American Psychiatric Association, 1994. Diagnostic and Statistical Manual of Mental Disorders, 4th ed. American Psychiatric Association, Washington, DC.

Anderson, D.N., Abou-Saleh, M.T., Collns, J., Hughes, K. Catteli, R.J., Hamon, C.G.B., Blair, J.A., Dewey, M.E., 1992 Pterin metabolism in depression: an exiension of the amine hypothesis and possible marker of response to ECT. Psychol. Med. 22, 863-869.

Bridges, A.J., 1993. Fibromyalgia, antinuclear antibodies and Bridges, A.J., 1993. Fibromyalgia, antinuclear antibodies and
clinical features of connective tissue diseise (letter). Clin. Exp. clinical features of connective
Rheumitol. 11, 696-697.

Bridges, A.J., Anderson, J.D., Burns, D.E., Kemple, K., Kaplan, J.D., Lorden, T., 1996. Autoantibodies in patients with silicon implants. Curr. Top. Microbiol. Immunol. 210, 277-282.

Clauw, D.J., 1995. The pathogenesis of chronic pain and fatigue syndrornes, with special reference to fibromyalgia. Med. Hypoth. 44, 369-378.

Duch, D.S., Woolf, J.H., Nichol, C.A., Jonathan, R.D., Garbutt, J.C., 1983. Urinary excretion of biopterin and neopterin in psychiatry disorders. Psychiatr. Res. 11, 83-89.

Dunbar, P.R., Hill, J., Neale, T.J., Mellsop, G.W., 1992. Neopterin measurement provides evidence of altered cell-mediated immeasurement provides evidence of altered cell-mediated im-
munity in patients with depression, but not with schizophrenia. munity in patients with depressi
Psychol. Med. 22, 1051-1057.

Giovannoni, G., Lai, M., Kidd, D., Thorps, J.W., Miller, D.H., Thompson, A.J., Kleir, G., Feldmam, M., Thompson, E.J. 1997. Daily urinary neopterin excretion as an immunological marker of disease activity in multiple sclerosis. Brain 120, $1-13$.

Goldenberg. D.N., 1989. Management of fibromyalgia syndrome Rheum. Dis. Clin. North Am. 15, 499-512.

Hamilton, M. 1960. A rating scale for depression. J. Neurol Neurosurg. Psychiatry 23, 56-61.

Hawley, D.J., Wolfe, F., 1993. Depression is not more common in theumatoid arthritis: a 10-year longitudinal study of 6153
anley theumatoid arthritis: a 10-year longitudinal study of 6153
patients with theumatic disease. J. Rheusnatol. 20, 2025-2031 patients with theumatic disease. J. Rheurnatol. 20, 2025-2031.
Hench, P.K., Mitler, M.M., 1986. Fibromyalgia: 2. Management guidelines and research findings. Postgrad. Med. 80, 57-69.

Hernanz, W., Valenzuela, A., Quijada, J., Garcia, A., De La Iglesia, J.L., Gutierrez, A., Povedano. J., Moreno, I., Sanchez, B., 1994. Lymphocyte subpopulations in patients with primary fibromyalgia. J. Rheumatol. 21, 2122-2124.

Heyes, M.P., Quearry, B.J., 1991. Incre ised L-tryptophan, 5hydroxyindoleacetic acid, 3-hydroxykynurenine and quinolonic acid concentrations in cerebral corte: following systemic endotoxin administration Adv. Exp. Mes Biol. 294. 559-562

Hu, B., Hissong, B.D. Carlin, J.M., 1995. Interleukin-1 enhances indolearnine 2,3-dioxygenase activity by increasing specific mRNA expression in human mononjclear phagocytes. J. mRNA expression in human m
Interferon Cyt. Res. 15, 617-624.
Hudson, J.I., Hudson, M.S., Pliner, L.F., Goldenberg, D.L., Pope Jr. H.G., 1985. Fibromyalgia and major affective disorder: a controlled phenomenology and family history study. Am. J. controlled phenomenology
Psychiatry 142, 44!-446.

Jacobsen. S., Jensen, K.E., Thomsen, C., Danneskiold-Samsoe, B. Henriksen, O., 1992. 31-P magnetic resonance spectroscopy of skeletal muscle in patients with fibromyalgia. J. Rheumatol. 19 $1600-1603$

Jacobsen, S., Gam, A., Egsmose, C., Olsen, M., DanneskioldSamsue, B.. Finn Jensen, G., 1993. Bone mass and turnover in fibronyalgia. J. Rheumatol. 20, 856-858.

Joyce, P.R., Hawes, C.R., Mulder, R.T., Sellman, J.D., Wilson, D.A., Boswell, D.R., 1992. Elevated levels of acute phase plasma proteins in major depression. Biol. Psychiatry 32 1035-1041.

Klein, R., Bansch, M., Berg, P.A., 1992. Clinical relevance of antibodies against serotonin and gangliosides in patients with antibodies against serotonin and gangliosides in patients with
primary fibromyalgia syndrome. Psychoneuroendocrinology primary fibro $17,593-598$.

Klein, R., Berg, P.A., 1994. A comparative study on antibodies to nucleoli and 5 -hydroxytryptamine in patients with fibromyalgia syndrome and tryptophan-induced eosinophilia-myalgia syndrome. Clin. Invest. 72, 541-549.

Krag, N.J., Norregaard, J., Larsen, J.K., Danneskiold-Samsoe, B. 1994. A blinded controlled evaluation of anxiety and depressive symptoms in patients with fibromyalgia, as measured by standardized psychometric interview scales. Acta Psychiatr. Scand. 89, 370-375.

Lijnen, P., Hespel, P.. Vanden Eynde, E., Amery, A., 1985. Urinary excretion of electrolytes during prolonged physical activity in normal man. Eur. J. Appl. Physiol. 53, 317-321.

Maes, M., 1995. Evidence for an immune response in major depression: a review and hypothesis. Prog. Neuro-Psychopharmacol. Biol. Psychiatry 19, 11-38.

Maes, M., Scharpe, S., Cooremans, W., VanGrootel, L., Lyttenbrouck, W., Minner, B., Suy, E., Cosyns, P., 1992. Higher al-antitrypsine, haptoglobin, and ceruloplasmin, and lower retinol binding protein plasma levels in major depressed subjects. J. Affect. Disord. 24, 183-192.

Maes, M., Meltzer, H.Y., Stevens, W., Cosyns, P., Blockx, P., 1994. Multiple reciprocal relationships between in vivo cellular immunity and hypothalamic-pituitary-adrenal axis in depresimmunity and hypothalamic-pituitary-
sion. Psychol. Med. 24, 167-177.

Maes, M. Scharpé, S., Meltzer, HY, Okayli, G., Bosmans, E. Maes. M., Scharpé, S., Meltzer, H.Y., Okayli, G., Bosmans, E..
D'Hondt, P., Yanden Bossche, B., Cosyns, P. 1994. Increased neopterin and interferon-g secretion and lower availability of L-tryptophan in major depression: further evidence for activition of cell-mediated immunity. Psychiatr. Res. 54, 143-160. Maes, M., Bosmans, E., Meltzer, H.Y., 1995. Irnmunoendocrine aspects of major depression: relationships berween plasm interleukin- 6 and solubie interleukin-2 receptor, prolactin and cortisol. Eur. Arch. Psychiatr. Clin. Neurosci. 245, 172-178.

Maes, M.. Smith, R., Scharpe, S., 1995. The monocyte-T-lymphocyte hypothesis of major depression. Psychoneuroendocrinology 20, $111-116$.

Maes, M., Bosmans, E., De Jongh, R., Kenis, G., Vandoolaeghe, E., Neels, H., 1997. Increased serum IL-6 and IL-1 receptor antagonist concentrations in major depression and treatment resistant depression. Cytokine, in press. 
Motyl, T., Traczyk, Z., Holska, W., Daniewska-Michalska, D., Ciesluk, S., Kukulska, W., Kaluzny, Z., Podgurniak, M., 1993. Comparison of urinary neopterin and pseudouridine in patients with malignant proliferative diseases. Eur. J. Clin. Chem. Clin. Biochem. 31, 205-209.

Nichol, C.A., Smith, G.K., Duch, D.S., 1985. Biosynthesis and metabolism of tetrahydrobiopterin and molybdopterin. Annu. Rev. Biochem. 54, 729-764.

Puttini. P.S.. Caruso, I., 1992. Primary fibromyalgia syndrome and 5-hydroxy-L-tryptophan: a 90-day open study. J. Int. Med. Res. 20. 182-189.

Russell, I.J., 1989. Neurohormonal asfects of fibromyalgia syndrome. Rheum. Dis. Clin. North Am. 15, 149-165.

Russell, 1.J., Michalek, J.E., Vipraio, G.A., Fletcher, E.M., Javors, M.A., Bowden, C.A., 1991. Platelet 3-H imipramine uptake receptor density and serum serotonin levels in patients with fibromyalgia/fibrositis syndrome. J. Rheumatol 19, 104-109. accomani, L., Vigliarolo, M.A., Sbolgi, P., Ruffa, G., Dori Lamba, L., 1993. Sindrome fibromialgica giovanile: due
clinici. Ped. Med. Chir. (Med. Surg. Ped.) 15, 99-101.

Saito, K., Markey, S.P., Heyes, P., 1992. Effects of immune activation on quinolinic acid and neurjactive kynurenines in the mouse. Neuroscience 51, 25-39.

Seidel, A., Arolt, V., Hunstiger, M., Rink, L., Behnisch, A. Kirchner, H., 1996. Increased CD56 + natural killer cells and related cytokines in major depression. Clin. Immunol. Immunopathol. 78, 83-85.

Sluzewska, A., Rybakowski, J., Bosmans, E., Sobieska, M., Berghmans, R., Maes, M., Wiktorwicz, K., 1996. Indicators of imrnume activation in major depression. Psychiatr. Res. 64, 161-167.
Smith, R.S., 1991. The macrophage theory of depression. Med. Hypoth. 35, 298-306.

Song, C., Dinan, T., Leonard, B.E., 1994. Changes in immunoglobulin, complement and acute phase protein levels in the depressed patients and normal controls. J. Affect. Disord. 30. 283-288.

Takikawa, O., Yoshida, R., Yasui, H., Hayaishi, O., 1984. The relationship between plasma kynurenine and indoleamine 2,3dioxygenase activity in the extrahepatic tissues. In: Schlos sberger, H.G., Kochen. W., Linzen, B., Steinhart, H. (Eds.), Progress in Tryptophan and Serotonin Research. Walter de Gruyter, Berlin-New York, pp. 517-520.

Van Acker, K.J., Eyskens, F.J., Verkerk, R.M., Scharpé, S., 1993. Urinary excretion of purine and pyrimidine metabolites in the neonate. Pediatr. Res. 34, 762-766.

Wolfe, F., Smythe, H.A., Yunus, M.B., Bennett, R.M., Bombardier, C., Goldenberg, D.L., Tugwell, P., Campbell, S.M., Abeles, M., Clark, P. et al., 1990. The American College of Rheumatology 1990: Criteria for the Classification of Fibmemyalgia. Report of the Multicenter Criteria Committee. Arthr. Rheumatol. 33, 160-172

Yunus, M.B., Dailey, J.W., Aldag, J.C., Masi, A.T., Jobe, P.C. 1992. Plasma tryptophan and other amino acids in primary fibromyalgia: a controlled study. J. Rheumatol. 19, 90-94. Yunus, M.B., Hussey, F.X., Aldag, J.C., 1993. Antinuclear antibodies and connective tissue disease features in fibromyalgia syndrome: a controlled study. J. Rheumatol. 20, 15571560 . 


\title{
Chapter 3
}

\section{Serotonin-immune interactions in elderly volunteers and in patients with Alzheimer's disease (DAT): Lower plasma tryptophan availability to the brain in the elderly and increased serum interleukin-6 in DAT}

\author{
S. Bonaccorso ${ }^{1,2}$, A. Lin ${ }^{1}$, C. Song ${ }^{1,3}$, R. Verkerk ${ }^{4}$, G. Kenis ${ }^{5}$, E. Bosmans 5 , S. Scharpe ${ }^{4}$, \\ M. Vandewoude ${ }^{6}$, A. Dossche ${ }^{6}$, and M. Maes ${ }^{1,7}$ \\ ${ }^{1}$ Clinical Research Center for Mental Health, Antwerp, Belgium; ${ }^{2}$ Department of Psychiatry, University of \\ Roma "La Sapienza", Roma, Italy; ${ }^{3}$ Life Sciences Research Center, Carleton University, Ottawa, Canada; \\ ${ }^{4}$ Department of Medical Biochemistry, University of Antwerp, Antwerp, Belgium; ${ }^{5}$ Eurogenetics, \\ Tessenderlo, Belgium; ${ }^{6}$ Department of Gerontology and Geriatrics, University of Antwerp, Wilrijk, Belgium; \\ ${ }^{7}$ Department of Psychiatry, Vanderbilt University, Nashville, Tennessee, U.S.A.
}

\begin{abstract}
The aims of this study were to examine the plasma availability of tryptophan, the precursor of 5-hydroxytryptamine $(5-H T)$, and serum cytokines, such as interleukin-6 (IL-6) and $I L-8$, in normal elderly volunteers and in patients with Alzheimer's disease (DAT). Elderly norma volunteers (mean age $=78.3 \pm 5.7$ years) had a sig nificantly lower tryptophan/competing amino acids (valine+leucine +isoleucine+phenylala nine+tyrosine) ratio than younger subjects (mean " age $=32.9 \pm 8.1$ years). In normal volunteers, there were significant and inverse relationships between age and either plasma tryptophan or the tryptophan/competing amino acids ratio, and between the availability of tryptophan to the brain and serum IL-6 or IL-8. DAT patients had signifi- . cantly higher serum IL-6, but not IL-8, than agematched normal volunteers. There were no significant differences in the availability of tryptophan to the brain between DAT patients and agematched normal volunteers. The results suggest that: 1) in normal humans, the availability of plasma tryptophan to the brain decreases with age, and with activation of the immune system; and 2) increased production of IL-6 may play a role in the pathogenesis of DAT.

(Aging Clin. Exp. Res. 10: 316-323, 1998)

○1998, Editrice Kurtis
\end{abstract}

\section{INTRODUCTION}

There is now evidence that alterations in the peripheral and central metabolism of serotonin (5-HT), as well as the immune-inflammatory responses may play a role in Alzheimer's disease (DAT), and the normal aging process. Many studies have documented serotonergic abnormalities in the brain of DAT patients. These include: i) reductions in brain and cerebrospinal fluid (CSF) 5-HT and/or 5-hydroxyindolacetic acid (5-HIAA), the major 5-HT metabolite, in some but not all studies (1-7); ii) cell loss and tangle formation in the dorsal raphe nucleus $(8,9)$; and iii) a loss of presynaptic 5-HT terminals and reductions in brain 5-HT1 and 5-HT2 receptor binding $(6,10-$ 12). Disorders in 5-HT may be involved in the cognitive (13) as well as the non-cognitive symptoms of DAT, e.g., behavioral symptoms and depression (14). Declines in serotonergic cell numbers in the raphe nuclei, and the occurrence of aberrant serotonergic fibers may be implicated in the normal aging process (15). Neurochemical data on the serotonergic system in relation to the normal aging process are more controversial. Unaltered, increased or decreased 5-HT or 5-HIAA concentrations were found in selected brain regions of elderly persons $(15-20)$. There is also some evidence that the availability of plasma tryptophan, the precursor of 5-HT, as indicated by the ratio of plasma tryptophan to the sum of amino acids

Key words: Alzheimer's disease, cytokines, dementia, interleukin-6, interleukin-8, tryptophan.

Correspondence: M. Maes, M.D., Ph.D. Clinical Research Center for Mental Health (CRC-MH), University Department of Psychiatry, AZ Stuivenberg, 267 Lange Beeldekensstraat, 2060 Antwerp, Belgium.

Received October 21, 1997; accepted in revised form February 4, 1998. 
lown to compete for the same cerebral uptake lechanism (i.e., the competing amino acids valine, ucine, tyrosine, phenylalanine, and isoleucine) (21), lay gradually decrease with age in depressed subjects :2). Total and free tryptophan, as well as the trypsphan/competing amino acid ratio are indicators or the availability of tryptophan to the brain (23, 4) and, hence, for 5-HT synthesis in the brain (25). An immune-inflammatory response has been sugested to play a role in the pathophysiology or pathoenesis of DAT (26). There is evidence that an acute hase response in the brain, and increased cytokine roduction are involved in the neurodegenerative rocesses in DAT $(27,28)$. Elevated levels of pro-inlammatory cytokines, such as interleukin-1 (IL-1), $L-6$, and tumor necrosis factor, have been found in he brains, CSF or sera of DAT patients (29-32).

Aging may be associated with a progressive decline $n$ immune responsiveness to exogenous antigens, in increasing incidence of autoimmune reactions, ind an altered pattern of cytokine secretion (33). I was shown that serum IL- 6 concentrations rise with idvancing age in healthy elderly persons, and in old hesus monkeys (34). Mitogen-stimulated peripheral lood mononuclear cells of elderly subjects secrete ligher amounts of IL- 6 , IL- $1 \beta$ and TNF $\alpha$ than those ff young subjects (35). However, other reports demon;trate that functionally intact immune responses can be rresent in the elderly. For example, although there was a clear age-dependent increase in the prevaence of organ-specific antibodies in sera from elder $y$ subjects (i.e., 70-85 years), the prevalence of these tutoantibodies was not significantly altered in centezarians (36). Other data suggest that various immune unctions are kept at a high level of efficiency in cenenarians (37). This is important because healthy zentenarians constitute an example of successful aging (38). Therefore, it was suggested that alterations in cell-mediated and humoral immunity observed in the ilderly are not the consequence of the aging process itself, but rather might be related to age-associated disease (39).

It is known that an immune-inflammatory response, with an increased production of pro-inflammatory cytokines, is accompanied by decreased plasma total tryptophan (40). This phenomenon may be caused by 1) induction of indoleamine 2,3 dioxygenase (IDO), a major tryptophan catabolizing enzyme, by cytokines, such as interferon- $\gamma$ (IFN $\gamma$ ), IL-1 and IL-2 (40-47); and 2) muscle breakdown of proteins, increased release of amino acids, inhibited muscle amino acid uptake, and increased use of some amino acids for leukocyte activity and synthesis of acute phase proteins $(48,49)$. In major depression and detoxified chronic alcoholic patients without apparent liver disease, significant inverse correlations were found between the availability of tryptophan to the brain and markers of immune activation, such as increased serum neopterin IL- 6 and IL-8, and lower albumin and transferrin concentrations (50-53).

The aims of the present study were: 1) to examine the availability of tryptophan to the brain and serum immune-inflammatory markers, such as IL- 6 and IL- 8 , in DAT patients and in the elderly; and 2) to determine whether there are inverse relationships between the availability of tryptophan to the brain and serum IL-6 or IL-8 in DAT patients or elderly normal volunteers.

\section{SUBJECTS AND METHODS}

\section{Subjects}

Forty-six subjects participated in the present study i.e., 31 normal volunteers and 15 DAT patients. Normal subjects were excluded for a present or lifetime history of DSM-III-R axis I diagnoses, such as organic mental disorder, substance use disorder, anxiety disorders, major depression, and bipolar disorder. Not one was a regular drinker or had ever been taking psychotropic drugs. The normal volunteers had a female/male ratio of $17 / 14$, and a mean $\pm \mathrm{SD}$ age of $55.2( \pm 22.2)$ years (range: $22-91$ years). The study group of normal volunteers was divided in two subgroups: 1) a "young" study group $(\mathrm{N}=13)$ with a mean \pm SD age of $32.9( \pm 8.1)$ years (range: $22-45$ years) and a female/male ratio of $5 / 8$; and 2 ) an "elderly" study group ( $\mathrm{N}=13$ ) with a mean $\pm \mathrm{SD}$ age of $78.3( \pm 5.7)$ years (range: $70-91)$ and a female/male ratio of $7 / 6$

DSM-III-R criteria (54) were used to make the diagnosis of DAT. All DAT subjects had a CT scan which was negative for multi-infarct dementia, and a score of $<16$ on the Mini Mental State Examination. The mean \pm SD age of the DAT patients was 78.4 $( \pm 10.3)$ years (range: $56-94$ years) and the female/male ratio was $12 / 3$. All DAT patients were free of psychotropic drugs. Fifteen of the 31 normal volunteers included in this study served as an agematched control group for the DAT patients. The mean $\pm \mathrm{SD}$ age of this normal control group was 75.6 $( \pm 9.1)$ years (range: $53-91$ years) and the female/male ratio was $7 / 8$

All subjects (DAT patients and normal volunteers) had normal physical examination as screened by a senior gerontologist (MVDW), a normal electrocardiogram, and normal values of blood results, such as hematologic measures (e.g., hematocrit), plasma vitamin B12, folic acid, thyroid hormones and thyroid stimulating hormone, electrolytes, SGOT, SGPT and 
$\gamma \mathrm{GT}$, and blood renal tests (blood urea and creatinine). We also excluded subjects (either in the DAT or normal control group) with disorders which are often accompanied by mental deterioration, e.g., Parkinson's disease, Huntington's disease and chronic alcoholism. All subjects were free of 1) chronic illnesses known to affect either the endocrine-immune status or plasma tryptophan availability to the brain; and 2) acute infectious or allergic reactions for at least two weeks prior to the study.

\section{Methods}

In normal volunteers and DAT patients blood samples were taken at 7:45 ( $\pm 30 \mathrm{~min})$ after an overnight fast. Sera and plasma samples were kept at $-75^{\circ} \mathrm{C}$ until thawed for assay. All blood specimens for the assay of plasma amino acids and serum IL- 6 or IL-8 in DAT patients and normal volunteers were assayed in a single run, with a single lot number of reagents and consumables employed by a single operator (A.L.). Plasma amino acids were determined by means of an HPLC method, as described previously $(55,56)$. The intra-assay $C V$ values obtained in our laboratory are: tryptophan 3.3\%; tyrosine $3.8 \%$; valine $3.0 \%$; phenylalanine $3.2 \%$; isoleucine $3.4 \%$; and leucine $3.7 \%$. The L-TRP/valine+leucine+isoleucine+tyrosine+phenylalanine ratio was computed and multiplied by 100 . Serum IL- 6 and IL-8 were determined with an ELISA method (Eurogenetics, Tessenderlo, Belgium) based on appropriate and validated sets of monoclonal antibodies. The intraassay CV values were less than $8 \%$.

\section{Statistics}

Group mean differences were assessed using analyses of variance (ANOVAs). In order to control for pos- sible effects of gender (differences between the young and elderly study groups), and age and gender (differences between normal elderly volunteers and DAT patients), we used analyses of covariance (ANCOVAs), with age and/or gender as covariates. Relationships between variables were assessed by means of Pearson's product moment correlations, or through multiple regression analyses. Box-Cox transformations were used either to normalize the distribution of the variables (IL- 6 and IL-8), or adjust for heterogeneity of variance between the study groups.

\section{RESULTS}

Younger and elderly normal volunteers

There were significant differences in age between the young and elderly study groups $(F=273, d f=1 / 24$, $p<10^{-4}$ ); there were no significant differences in the female/male ratio $\left(\chi^{2}=0.1 \mathrm{df}=1, p=0.7\right)$. Table 1 shows the amino acid measurements in younger vs elderly normal volunteers. There was a trend toward lower tryptophan in elderly than in younger normal volunteers. There were no significant differences in any of the five competing amino acids between both groups. The tryptophan/competing amino acids ratio was significantly lower in elderly normal volunteers than in younger normal volunteers. There were no significant differences in serum IL- 6 or IL- 8 between both study groups.

Table 2 lists the intercorrelation matrix between the plasma amino acids and age and gender (point-biserial correlation coefficients) in the total study group of normal volunteers. No significant correlations emerged between age and any of the amino acids, although there was a trend toward a negative correlation with tryptophan $(r=-0.30, p=0.09)$. There was a significant

Table 1 - Measurements of plasma amino acids, the tryptophan/competing amino acid (TRP/CAA) ratio and serum interleukin-6 (IL-6) and IL-8 in young vs elderly normal volunteers.

\begin{tabular}{llllllllll}
\hline Groups & Tryptophan & Tyrosine & Valine & Phenylalanine & Isoleucine & Leucine & $\begin{array}{l}\text { TRP/CAA } \\
\text { ratio } \times 100\end{array}$ & $\begin{array}{l}\text { IL-6 (pg/mL) } \\
\text { median (q25-q75) }\end{array}$ & $\begin{array}{l}\text { IL-8 }(\mathrm{pg} / \mathrm{mL}) \\
\text { median (q25-q5) }\end{array}$ \\
\hline Young & $78(16)$ & $69(13)$ & $253(55)$ & $93(17)$ & $81(19)$ & $156(36)$ & $12.1(2.0)$ & $1.0(0.8-7.2)$ & $20.9(5.7-143)$ \\
Elderly & $66(10)$ & $73(20)$ & $234(26)$ & $97(11)$ & $78(11)$ & $151(23)$ & $10.5(1.1)$ & $3.4(2.3-9.0)$ & $9.8(6.5-24.2)$ \\
$\mathrm{F}^{*}$ & 4.0 & 0.4 & 0.7 & 0.7 & 0.00 & 0.00 & 5.7 & 0.7 & 0.9 \\
$\mathrm{df}$ & $1 / 23$ & $1 / 23$ & $1 / 23$ & $1 / 23$ & $1 / 23$ & $1 / 23$ & $1 / 23$ & $1 / 23$ & $1 / 23$ \\
$p$ & 0.055 & 0.5 & 0.6 & 0.6 & 0.99 & 0.92 & 0.02 & 0.6 & 0.7 \\
\hline
\end{tabular}

All results are shown as mean $\pm \mathrm{SD}$, except IL- 6 and IL- 8 : median and $\mathrm{q} 25$ and $\mathrm{q} 75$ values. Amino acids are expressed in $\mu \mathrm{mol} / \mathrm{L}$

F: All results of ANCOVAs with gender as covariate. 
Table 2 - Intercorrelation matrix between plasma tryptophan, the five competing amino acids, age and sex in 31 normal volunteers (age range: $22-91$ years).

\begin{tabular}{|c|c|c|c|c|c|c|}
\hline Variables & Tyrosine & Valine & Tryptophan & Isoleucine & Phenylalanine & Leucine \\
\hline Valine & $0.39^{*}$ & & & & & \\
\hline Tryptophan & $0.42^{*}$ & $0.62^{* * *}$ & & & & \\
\hline Isoleucine & $0.37^{*}$ & $0.94^{* * *}$ & $0.60^{* * *}$ & & & \\
\hline Phenylalanine & $0.59^{* * *}$ & $0.62^{* * *}$ & $0.35^{*}$ & $0.63^{* * *}$ & & \\
\hline Leucine & $0.44^{*}$ & $0.90^{* * *}$ & $0.60^{* * *}$ & $0.94^{* * *}$ & $0.77^{* * *}$ & \\
\hline Age & 0.13 & -0.09 & -0.30 & 0.01 & 0.20 & -0.01 \\
\hline Gender ${ }^{\mathrm{a}}$ & 0.04 & $0.46^{* *}$ & $0.37^{*}$ & $0.54^{* *}$ & 0.19 & $0.48^{* *}$ \\
\hline
\end{tabular}

and inverse correlation between age and the tryptophan/competing amino acids ratio $(r=-0.42, p=0.01)$.

This inverse relationship remained significant after considering the effects of gender ( $\mathrm{F}=5.6, p=0.02)$. Plasma concentrations of valine, tryptophan, isoleucine, and leucine were significantly higher in males than in females.

In the total study group of normal volunteers, there were significant and inverse correlations between the tryptophan/competing amino acids ratio and serum IL-6 $(r=-0.53, p=0.002)$ and IL-8 $(r=-0.41, p=0.02)$, and between plasma tryptophan and serum IL-8 $(r=-0.42, p=0.02)$. There was a trend toward a significant inverse relationship between plasma tryptophan and serum IL-6 $(r=-0.34, p=0.06)$. Up to $47.5 \%$ of the variance in the tryptophan/competing amino acids ratio $(F=8.1, d f=3 / 27, p=0.0007)$ was explained by the multiple regression on age $(\mathrm{F}=7.2, p=0.001)$, IL-8 $(\mathrm{F}=5.6, p=0.02)$ and $\mathrm{IL}-6(\mathrm{~F}=3.2, p=0.08)$ (all three variables were negatively loaded; gender was not sig- nificant in this regression equation). Up to $41.3 \%$ of the variance in the tryptophan/competing amino acids ratio $(\mathrm{F}=9.8, \mathrm{df}=2 / 28, p=0.0008$ ) could be explained by the regression on IL- $8(\mathrm{~F}=12.1, p=0.002)$ and age $(F=11.8, p=0.002)$ (both variables were negatively loaded in the regression equation; gender was not significant). Up to $43.5 \%$ of the variance in plasma tryptophan $(\mathrm{F}=6.9, \mathrm{df}=3 / 27, p=0.002)$ could be explained by the regression on IL- $8(\mathrm{~F}=11.0, p=0.003)$, age $(\mathrm{F}=6.1, p=0.02)$ and gender $(\mathrm{F}=5.2, p=0.03)(\mathrm{IL}-8$ and age were negatively loaded in this regression equation, and gender positively).

\section{DAT patients and normal volunteers}

There were no significant differences in age between the normal volunteers and the DAT patients $(\mathrm{F}=0.6, \mathrm{df}=1 / 28, p=0.6)$, nor in the female/male ratio between DAT patients and the elderly study group $\left(\chi^{2}=2.3, \mathrm{df}=1, p=0.1\right)$. Table 3 shows the amino acid and cytokine measurements in DAT patients

Tabie 3 - Measurements of plasma amino acids, the tryptophan/competing amino acids (TRP/CAA) ratio and serum interleukin-6(IL 6) and IL-8 in patients with Alzheimer's disease (DAT) and in age-matched, elderly normal volunteers.

\begin{tabular}{llllllllll}
\hline Groups & Tryptophan & Tyrosine & Valine & Phenylalanine & Isoleucine & Leucine & $\begin{array}{l}\text { TRP/CAA } \\
\text { ratio X 100 }\end{array}$ & $\begin{array}{l}\text { IL-6 }(\mathrm{pg} / \mathrm{mL}) \\
\text { median (q25-q75) }\end{array}$ & $\begin{array}{l}\text { IL-8 }(\mathrm{pg} / \mathrm{mL}) \\
\text { median (q25-q75) }\end{array}$ \\
\hline Controls & $65(10)$ & $73(18)$ & $233(25)$ & $97(10)$ & $78(10)$ & $151(22)$ & $10.3(1.2)$ & $3.5(2.3-5.2)$ & $11.1(8.6-27.2)$ \\
DAT & $57(12)$ & $66(11)$ & $216(45)$ & $103(23)$ & $73(14)$ & $145(28)$ & $9.6(2.1)$ & $11.2(8.1-16.5)$ & $14.6(11.4-21.6)$ \\
F* & 2.3 & 1.2 & 1.0 & 0.9 & 0.4 & 0.2 & 0.7 & 9.7 & 0.8 \\
df & $1 / 25$ & $1 / 25$ & $1 / 25$ & $1 / 25$ & $1 / 25$ & $1 / 25$ & $1 / 25$ & $1 / 26$ & $1 / 26$ \\
$p$ & 0.1 & 0.3 & 0.3 & 0.6 & 0.6 & 0.7 & 0.6 & 0.004 & 0.6 \\
\hline
\end{tabular}

All results are shown as mean $\pm \mathrm{SD}$, except IL- 6 and $\mathrm{IL}-8$ : median and $\mathrm{q} 25$ and $\mathrm{q} 75$ values. Amino acids are expressed in $\mu \mathrm{mol} / \mathrm{L}$. $F^{*}:$ All results of ANCOVAs with age and gender as covariates. 
and normal volunteers. There were no significant differences either in plasma amino acids, including tryptophan, or in the tryptophan/competing amino acids ratio between both groups. Serum IL- 8 was not significantly different between DAT patients and normal volunteers. Patients with DAT had significantly higher serum IL-6 than normal volunteers. In DAT patients, no significant correlations were found between the tryptophan/competing amino acids ratio and either serum IL-6 $(r=-0.13, p=0.7)$ or IL-8 $(r=-0.35, p=0.2)$, nor between plasma tryptophan and either IL-6 $(r=0.00, p=0.99)$ or IL-8 $(r=-0.21, p=0.5)$. Adjusting for possible effects of age and gender did not change any of these results.

\section{DISCUSSION}

The first major finding of this study is that the availability of plasma tryptophan to the brain in normal humans is significantly and inversely related to increasing age and signs of immune activation, such as increased serum IL-6 and IL-8 concentrations. Indeed, elderly normal volunteers had a significantly lower availability of plasma tryptophan to the brain than young normal volunteers. We found that the effects of age and immune activation were additive, and independently from each other predicted lower plasma tryptophan availability. We previously reported a significant and inverse correlation between age and the tryptophan/CAA ratio, but not plasma tryptophan, in depressed patients (22). In the rodent, an age-related reduction of the plasma ratio of tryptophan to competing amino acids and cortical tryptophan was found in old animals (57). There are some reports that the serotonergic system in the brain is sensitive to aging (6). Since the availability of plasma tryptophan, in part, determines brain 5-HT synthesis (25), it could be suggested that lower plasmä tryptophan availability in elderly normal humans plays a role in the age-sensitivity of the central 5-HT system. However, the neurochemical data on the serotonergic system and aging are controversial (see Introduction). This may be explained by region-specific differences in the effects of age on 5-HT turnover (58). For example, age showed 2 significantly positive, and 2 significantly negative correlations with 5-HT concentrations in different brain areas (58). In the present study, no significant differences in any of the five competing amino acids could be found between young and elderly volunteers. Rudman et al. (59), on the other hand, observed a delayed plasma clearance of phenylalanine and tyrosine in healthy elderly subjects.

The inverse correlations between the availability of plasma tryptophan and serum IL-6 or IL-8 suggest that plasma tryptophan is highly sensitive to small and graded increases in immune-inflammatory responsiveness, as indicated by increased serum IL-6 and IL-8. IL-6 is a pleiotropic cytokine, which is a major immune and inflammatory mediator, and plays a pivota role in T and B lymphocyte proliferation or differentiation, $\mathrm{T}$ cell activation, hematopoiesis, prostaglandin secretion, regulation of antibody production, and the acute phase response (60). IL- 8 is produced by various cells, such as monocytes, macrophages, neutrophils, endothelial cells, fibroblasts and hepatocytes, after stimulation by IL- 1 and TNF $\alpha$. IL- 8 has a chemotaxic activity not only on neutrophils, but also on eosinophils, basophils, natural killer cells and lymphocytes (60). As summarized in the Introduction, immune activation or inflammation are accompanied by lowered plasma availability of tryptophan through, amongst other things, induction of IDO by cytokines, such as IFN $\gamma$ and IL-1. Thus, the inverse relationships between tryptophan and serum cytokines found in the present study suggest that lower plasma tryptophan availability is not only highly sensitive to increasing age, but also to activation of the inflammatory response system. We previously found relationships between lower plasma tryptophan availability, and activation of the inflammatory response system in depression, i.e., increased serum neopterin, haptoglobin and IL-6, and lower serum albumin and transferrin, and in detoxified chronic alcoholic patients, i.e., increased serum IL-6 and IL-8 (50-53)

In the present study, no significant effects of age on serum IL-6 or IL- 8 could be found in the normal volunteers. Previous research, on the other hand, showed that: 1) serum IL-6 rises with advancing age in healthy persons, rhesus monkeys and the rodent (review: 61); and 2) mitogen-stimulated peripheral blood mononuclear cells of elderly subjects secrete higher amounts of IL-6 than those of young subjects (35). Our results are in agreement with previous reports that immune functions may not be changed significantly in healthy elderly persons $(37,39)$.

We found no significant alterations in the availability of tryptophan to the brain in DAT patients. These results suggest that the well documented serotonergic abnormalities in the brain of DAT patients (see Introduction) are probably not related to changes in the availability of plasma tryptophan. Jorgenson et al. (62) observed a significantly lower tryptophan/competing amino acids ratio in patients with dementia (including DAT) than in normal volunteers. However, since the median age of their normal volunteers was only 38 years, the lower tryptophan availability in dementia patients us normal controls was probably due to an age-related decrease in tryptophan availability in the dementia patients. 
Commensurate with earlier studies, which described the implication of the inflammatory response system in the pathogenesis of DAT (26-28), we found signs of an inflammatory response in DAT patients, i.e., increased serum IL-6 concentrations. In this respect, it has been hypothesized that DAT may result from an autoimmune process, characterized by either local or systemic increases in IL- 6 production, which induces $\alpha 2$-macroglobulin to alter the processing of amyloid precursor protein and, consequently, the disposition of amyloid $\beta$ protein within the senile plaques $(29,63)$. Our results, which show that increased serum IL-6 is specific for DAT and not for the normal aging process, do not corroborate the hypothesis that an age-related increase in IL- 6 activity is involved in the pathogenesis of DAT (61).

\section{REFERENCES}

1. Carlsson A., Adolfsson R., Aquilonius S.M., Gottfries C.G. Oreland L., Svennerholm L., Winblad B.: Biogenic amines in
human brain in nomal aging, senile dementia, and chronic alcoholism. Adv. Biochem. Pharmacol. 23: 295-304, 1980.

2. Bareggi S.R., Franceschi M., Bonini L., Zecca L., Smime S.: Decreased CSF concentrations of homovanillic acid and aminobutyric acid in Alzheimer's disease. Arch. Neurol 39: 709-712, 1982.

3. Arai H., Kosaka K., Tizuka R.: Changes of biogenic amines and their metabolites in postmortem brains from patients with Alzheimer-type dementia. J. Neurochem. 43: 388-393, 1984

4. Volicer L., Langlais P.J., Matson W.R., Mark K.A., Gamache P.H.: Serotonergic system in dementia of the Alzheimer in cerebrospinal fluid. Arch. Neurol. 42: 1158-1161, 1985.

5. Sparks D.L., Markesbery W.R., Slevin J.T.: Alzheimer's disease: monoamines and spiperone binding reduced in nucleus basalis. Ann. Neurol. 18: 562-565, 1986.

6. Gottfries C.G.: Disturbance of the 5-hydroxytryptamine metabolism in brains from patients with Alzheimer's demen-
tia. J. Neural Trans. 30 (Suppl.): 33-43, 1990.

7. Tohgi H., Abe T., Takahashi S., Kimura M., Takahashi J., Kikuchi T.: Concentrations of serotonin and its related substances in the cerebrospinal fluid in patients with Alzheimer type dementia. Neuroscience 141: 9-12, 1992.

8. Mann D.M., Yates P.O.: Serotonin nerve cells in Alzheimer's disease. J. Neurol. Neurosurg. Psychiatry 46: 96-98, 1983.

9. Yamamoto T., Hirano A.: Nucleus raphe dorsalis in Alzheimer's disease: neurofibrillary tangles and loss of large neurons. Ann. Neurol. 17: 573-577, 1985

10. Bowen D.M., Allen S.J., Benton J.J., Goodhardt M.J., Haan E.A., Palmer A.M., Sims N.R., Smith C.C.T., Spillane J.A. Esiri M.M., Neory D., Snowdon J.J., Wilcock G.K., Davison A.N.: Biochemical assessment of serotonergic and cholinergic dysfunction and cerebral atrophy in Alzheimer's disease. $J$ Neurochem. 41: 266-272, 1983.

11. Perry E.K., Perry R.H., Candy J.M., Fairbairn A.F., Blessed G., Dick D.J., Tomlinson B.E.: Cortical serotonin-2 receptor binding abnormalities in patients with Alzheimer's disease: comparisons with Parkinson's disease. Neurosci. Lett. 51 353-357, 1984

12. Reynolds G.P., Arnold L., Rosser M.N., Iversen L.L., Mountjoy C.Q., Roth M.: Reduced binding of H-ketanserin to cortical 5HT receptors in senile dementia of Alzheimer type. Neurosci. Lett. 44: 47-52, 1984.

13. Cassel J.C., Jeltsch H.: Serotonergic modulation of cholinergic function in the central nervous system: cognitive implications. Neuroscience 69: 1-41, 1995.

14. Blessed G.: Clinical aspects of the senile dementias. In Roberts P.J. (Ed.), Biochemistry of dementia. Wiley, London, 1980, pp. 1-14.

15. Marielle G., van Luijtelaar P.A., Tonnaer J.A.D.M., Steinbusch H.W.M.: Aging of the serotonergic system in the rat forebrain an immunocytochemical and neurochemical study. Neurobiol. Aging 13: 201-215, 1992

16. Arranz B., Blennow K., Ekman R., Eriksson A., Mansson J.E. Marcusson J.: Brain monoaminergic and neuropeptidergic variations in human aging. J. Neural Transm. 103: 101-115 1996.

17. Godefroy F., Bassant M.H., Weil-Fugazza J., Lamour Y.: Age-related changes in dopaminergic and serotonergic in dices in the rat forebrain. Neurobiol. Aging 10: 187-190, 1990.

18. Timiras P.S., Hudson D.B., Segall P.E.: Lifetime brain serotonin: regional effects of age and precursor availability. Neurobiol. Aging 5: 235-242, 1984

19. Morettj A., Carfagna N., Trunzo F.: Effect of aging on monoamines and their metabolites in the rat brain. Neu rochem. Res. 12: 1035-1039, 1987.

20. Malhado A., Cano J., Santiago M.: The change with age in biogenic amines and their metabolites in the striatum of the rat. Arch. Gerontol. Geriatr. 5: 333-342, 1986.

21. Badawy A.A.B., Morgan C.J., Lovett J.W., Bradley D.M., Thomas R.: Decrease in circulating tryptophar availability to the brain after acute ethanol consumption by normal volunteers: implications for alcohol-induced aggressive behavior and depression. Pharmacopsychiatry 28: 93-97, 1995

22. Maes M., de Hert M., Claes R., Cosyns P., Suy E.: Serum L-tryptofaan en competitieve aminozuren bij depressieve patienten. Tijdschr. Psychiatrie 31: 267-283, 1989.

23. Curzon G., Sama G.S.: Tryptophan transport to the brain: newer findings and older ones reconsidered. In. Schlossberger H.G., Kochen W. Linzen B. Steinhart H. (Eds), Progress in Tryptophan and Serotonin Research Walter De Gruyter, Berlin, 1984, pp. 145-157.

24. Fernstrom J.D.: Tryptophan availability and serotonin synthesis in rat brain: effects of experimental diabetes. In: Schlossberger H.G., Kochen W., Linzen B., Steinhart H. (Eds.), Progress in Tryptophan and Serotonin Research. Walter de Gruyter, Berlin, 1984, pp. 161-172.

25. Moir A.T.B., Eccleston D.: The effect of precursor loading in the cerebral metabolism of 5-hydroxyindoles. $J$. Neurochem. 15: 1093-1108, 1986

26. Aisen P.S., Davis K.L.: Inflammatory mechanisms in Alzheimer's disease: implications for therapy. Am. J. Psychiatry 151: 1105-1113, 1994.

27. McGeer P.L., McGeer E.G.: The inflammatory response system of brain: implications for therapy of Alzheimer and other neurodegenerative diseases. Brain Res. Rev. 21: 195-218, 1995

Aging Clin. Exp. Res., Vol. 10, No. 4 
18. McRae A., Dahlstrom A.: Immune responses in brains of Alzheimer's and Parkinson's disease patients: hypothesis and reality. Rev. Neurosci. 3: 79-97, 1992.

9. Hull M., Strauss S., Berger M., Volk B., Bauer J.: The participation of interleukin- 6 , a stress-inducible cytokine, in the pathogenesis of Alzheimer's disease. Behav. Brain Res. 78 37-41, 1996 .

10. Fillit H., Ding W., Buee L., Kalman J., Altstiel L., Larlow B. Wolf-Klein G.: Elevated circulating tumor necrosis factor levels in Alzheimer's disease. Neurosci. Lett. 129: 318-320, 1991.

31. Blum-Degen D., Muller T., Kuhn W., Gerlach M., Przuntek H., Riederer P.: Interleukin- $1 \beta$ and interleukin- 6 are elevated in the cerebrospinal fluid of Alzheimer's and de novo Parkinso
ease patients. Neurosci. Lett. 202: 17-20, 1995.

32. Sheng J.G., Ito K., Skinner R.D., Mrak R.E., Rovnaghi C.R., van Eldik L.J., Griffin WST.: In vivo and in vitro evidence supporting a role for the inflammatory cytokine interleukin-1 as a driving force in Alzheimer pathogenesis. Neurobiol. Aging 17: 761-766, 1996.

3. Caruso C., Candore G., Cigna D., di Lorenzo G., Sireci G. Dieli F., Salerno A.: Cytokine production pathway in the elderly. Immunol. Res. 15: 84-90, 1996.

44. Ershler W.B., Sun W.H., Binkley N., Gravenstein S., Volk M.J., Kamoske G., Klopp R.G., Roecker E.B., Daynes R.A M.J., Kamoske G., Kopp R.G., Roecker E.B., Daynes R.A.,
Weindruch R.: Interleukin-6 and aging: blood levels and Weindruch R.: Interleukin- 6 and aging: blood levels and
mononuclear cell production increase with advancing age mononuclear cell production increase with advancing age and in vitro production is modifiable by dietary r
Lymphokine Cytokine Res. 12: 225-230, 1993.

15. Fagiolo U., Cossarizza A., Scala E., Fanalesbelasio E., Ortolani C., Cozzi E., Monti D., Franceschi C., Paganelli R.: Increased cytokine production in mononuclear cells of healthy elderly people. Eur. J. Immunol. 23: 2375-2378, 1993.

6. Mariotti S., Sansoni P., Barbesino G., Caturegli P., Monti D. Cossarizza A., Giacomelli T., Passeri G., Fagiolo U., Pinchera A.: Thyroid and other organ-specific autoantibodies in healthy centenarians. Lancet 340: 430, 1992.

37. Sansoni P., Cossarizza A., Brianti V., Fagnoni F., Snelli,G., Monti D., Marcato A., Passeri G., Ortolani C., Forti E.: Lymphocyte subsets and natural killer cell activity in healthy old
people and centenarians. Blood 82: 1601-1604, 1993.

38. Paganelli R., Scala E., Rosso R., Cossarizza A., Bertollo L., Barbieri D., Fabrizi A., Lusi E.A., Fagiolo U., Franceschi C. A shift to Th0 cytokine production by $\mathrm{CD} 4^{+}$cells in human longevity: studies on two healthy centenarians. Eur. J. Im munol. 26: 2030-2034, 1996.

39. Franceschi C., Monti D., Sansoni P., Cossarizza A.: The immunology of exceptional individuals: the lesson of centenarians. Immunol. Today 16: 12-16, 1995.

10. Maes M., Meltzer H.Y., Scharpe S., Bosmans E., Suy E., Minner B., de Meester I., Calabrese J., Vandervorst C., Raus J., Cosyns P.: Relationships between lower plasma L-tryptophan levels and immune variables in depression. Psychiatry phan levels and immune
Res. 49: 11-27, 1993.

11. Hu B., Hissong B.D., Carlin J.M.: Interleukin-1 enhances indoleamine 2,3-dioxygenase activity by increasing specific mRNA expression in human mononuclear phagocytes. J. Interferon Cytokine Res. 15: 617-624, 1995.

12. Brown R.R., Lee C.M., Kohler P.C., Hank J.A., Storer B.E, Sondel P.M.: Altered tryptophan and neopterin metabolism in cancer patients treated with recombinant interleukin-2. Can cer Res. 49: 4941-4944, 1989.
43. Takikawa O., Yoshida R., Yasui H., Hayaishi O.: The relationship between plasma kynurenine and indoleamine 2,3dioxygenase activity in the extrahepatic tissues. In: Schlossberger H.G., Kochen W., Linzen B., Steinhart H. (Eds.), Progress in Tryptophan and Serotonin Research. Walter de Gruyter, Berlin, 1984, pp. 517-520.

44. Moroni F., Russi P Gallo-Mezo M.A. Moneti G., Pellicciari R.: Modulation of quinolinic and kynurenic acid content in the rat brain: effects of endotoxins and nicotinylalanine. J. Neurochem. 57: 1630-1635, 1991.

45. Meyer K.C., Arend R.A., Kalayoglu M.V., Rosenthal N.S., Byme G.I., Brown R.R.: Tryptophan metabolism in chronic irflammatory lung disease. J. Lab. Clin. Med. 126: 530-540, 1995.

46. Taylor M.W., Feng G.S.: Relationship between interferongamma, indoleamine 2,3-dioxygenase, and tryptophan catabolism. FASEB J. 5: 2516-2522, 1991.

47. Werner-Felmayer G. Werner E.R. Fuchs D., Hausen A Reibnegger G., Wachter H.: Neopterin formation and tryptophan degradation by a human myelomonocytic cell line THP-1) upon cytokine treatment. Cancer Res. 50: 28632867, 1990.

48. Moldawer L.L., Georgieff M., Lundholm K.: Interleukin-1, tumour necrosis factor-alpha (cachectin) and the pathogenesis of cancer cachexia. Clin. Physiol. 7: 263-274, 1987.

49. Hasselgren P.O., Pedersen P., Sax H.C., Warner B.W., Fischer J.E.: Current concepts of protein turnover and amino acid transport in liver and skeletal muscle during sepsis. Arch. Surg. 123: 992-999, 1988.

50. Maes M., Scharpe S., Meltzer H.Y., Okayli G., Bosmans E., D'hondt P., Vanden Bossche B., Cosyns P.: Increased neopterin and interferon $\gamma$ secretion and lower availability of neopterin and interferon $\gamma$ secretion and lower availability of L-tryptophan in major depression: further evidence for acti-
vation of cell-mediated immunity. Psychiatr. Res. 54: 143vation of cell
$160,1994$.

51. Maes M., Wauters A., Verkerk R., Neels H., Vangastel A., Cosyns P., Scharpe S., Desnyder R.: Lower L-tryptophan availability in depression: a marker of a more generalized disorder in protein metabolism. Neuropsychopharmacol. disorder in protein

52. Maes M., Verkerk R., Vandoolaeghe E., Van Hunsel F., Neels H., Wauters A., Demedts P., Scharpe S.: Serotoninimmune interactions in major depression: lower serum tryptophan as a marker of an immune-inflammatory response. Eur. Arch. Psychiatry Clin. Neurosci. 247: 154-161, 1997.

53. Maes M., Lin A., Bosmans E., Vandoolaeghe E., Bonaccorso S., Kenis G., de Jongh R., Verkerk R., Scharpe S.: Serotoninimmune interactions in detoxified chronic alcoholic patients without apparent liver disease: indicants of immune activation and lower plasma total tryptophan. Psychiatr. Res. 78: 151161, 1998.

54. American Psychiatric Association: Diagnostic and Statistical Manual of Mental Disorders. American Psychiatric Association, Washington, 1987

55. Turnell D., Cooper J.: Rapid assay for amino acids in serum or urine by precolumn derivatisation and reversed-phase liquid chromatography. Clin. Chem. 28: 527-531, 1982.

56. Maes M., Schotte C., Scharpe S., Martin M., Blockx P.: The effects of glucocorticoids on the availability of L-tryptophan and tyrosine in the plasma of depressed patients. J. Affect. Disord. 18: 121-127, 1990. 
7. Yeung J.M., Friedman E.: Effect of aging and diet restriction on monoamines and amino acids in cerebral cortex of Fischer-34 rats. Growth Dev. Aging 55: 275-282, 1991.

8. Bucht G., Adolfsson R., Gottfries C.G., Roos B-E., Winblad B. Distribution of 5-hydroxytryptamine and 5-hydroxyindoleacetic acid in human brain in relation to age, drug influence, agonal acid in human brain in relation to age, drug influence, agona
status and circadian variation. J. Neural. Transm. 51: 185203, 1981

59. Rudman D., Abbasi A.A., Chaudry F., Mattson D.E.: Delayed plasma clearance of phenylalanine and tyrosine in elderly men. J. Am. Geriatr. Soc. 39: 33-38, 1991.
60. Cavaillon J-M.: Les cytokines. Masson, Paris, 1996

61. Ershler W.B.: Interleukin-6: A cytokine for gerontologists. Geriatr. Biosci. 41: 176-181, 1993.

62. Jorgenson S., Bille A., Andersen J., Jensen H.V., Olafsson K., Arup P., Moller S.E.: Fluvoxamine treatment of dementia: tryptophan levels. Biol. Psychiatry 34: 587-588, 1993

63. Vandenabeele P., Fiers W.: Is amyloidogenesis during Alzheimer's disease due to an IL-1/1L-6-mediated "acute phase response" in the brain. Immunol. Today 12: 217 219, 1991. 


\section{Chapter 4}

\section{Psychological and behavioural effects of interferons Stefania Bonaccorso ${ }^{a, b, c}$, Herbert Meltzer ${ }^{c}$ and Michael Maes ${ }^{b, c}$}

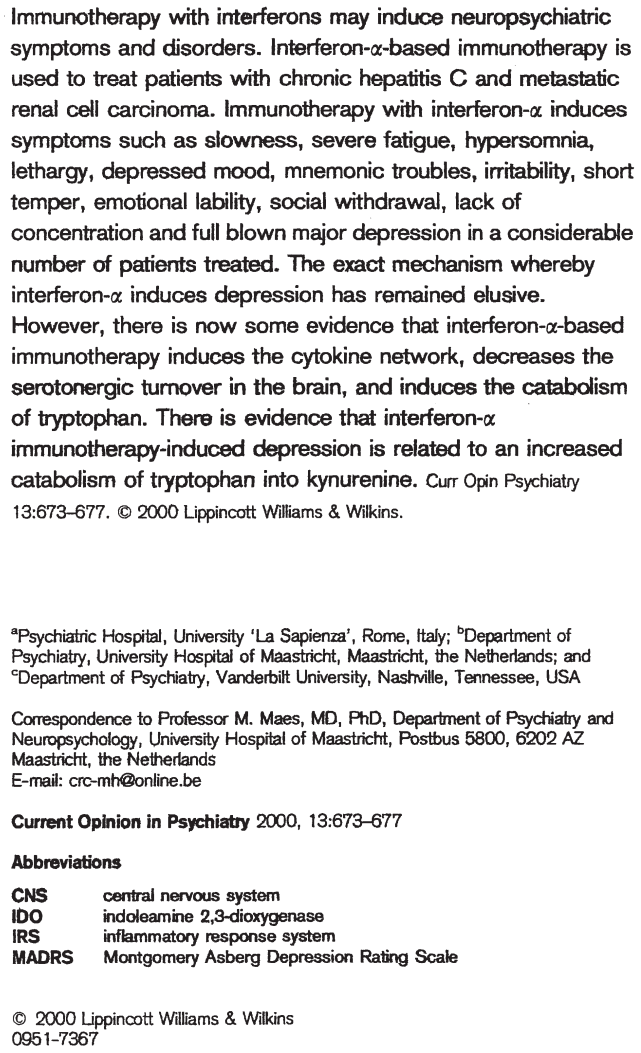
symptoms and disorders. Interferon- $\alpha$-based immunotherapy is used to treat patients with chronic hepatitis $\mathrm{C}$ and metastatic renal cell carcinoma. Immunotherapy with interferon- $\alpha$ induces symptoms such as slowness, severe fatigue, hypersomnia, lethargy, depressed mood, mnemonic troubles, irritability, short temper, emotional lability, social withdrawal, lack of concentration and full blown major depression in a considerable number of patients treated. The exact mechanism whereby interferon- $\alpha$ induces depression has remained elusive. However, there is now some evidence that interferon- $\alpha$-based immunotherapy induces the cytokine network, decreases the serotonergic turnover in the brain, and induces the catabolism of tryptophan. There is evidence that interferon- $\alpha$ immunotherapy-induced depression is related to an increased catabolism of tryptophan into kynurenine. Curr Opin Psychiatry 13:673-677. @ 2000 Lippincott Williams \& Wilkins.

'Psychiatric Hospital, University 'La Sapienza', Rome, Italy; 'Department of Psychiatry, University Hospital of Maastricht, Maastricht, the Netherlands; and

Correspondence to Professor M. Maes, MD, PhD, Department of Psychiatry and Neuropsychology, University Hospital of Maastricht, Postbus 5800, 6202 AZ Maastricht, the Netherland

E-mail: crc-mh@online.be

Current Opinion in Psychiatry 2000, 13:673-677

Abbreviations

CNS central nervous system

IDO indoleamine 2,3-dioxygenase

MADRS Montgomery Asberg Depression Rating Scale

(C) 2000 Lippincott Williams \& Wilkins (c) 2000 Lip

\section{Introduction}

Immunotherapy with IFN- $\alpha$ is used to treat patients with chronic hepatitis $\mathrm{C}$ and $\mathrm{B}$, , metastatic renal cell carcinoma brain tumours and other virus infections such as natural colds, papilloma virus, condyloma acuminata and common warts. IFN- $\alpha$ (or leukocyte interferon) is a cytokine produced by lymphocytes, null lymphocytes and macrophages. It has pleiotropic functions that include potent antiviral and antiproliferative effects . Immunotherapy with IFN- $\alpha$ produces a high incidence of adverse effects, including psychiatric complications.

Commonly experienced adverse effects are a 'flu-like syndrome with myalgias, fever, chills, arthralgias and headaches, which occur normally a few hours after administration . Most studies initially focused on evaluating neurological and neuropsychological functions such as memory, visuospatial and visuoconstruction ability by means of the Wechsler memory scale, and cognitive tests, such as the WAIS-R. These tests showed that immunotherapy with IFN- $\alpha$ is accompanied by cognitive deterioration, suggesting frontal-subcortical cerebral dysfunction.

\section{Psychiatric side-effects of interferon- $\alpha$-based} immunotherapy

The neuropsychiatric complications consist mainly of slowness, severe fatigue, hypersomnia, lethargy, depressed mood, irritability, short temper, emotional lability, social withdrawal and lack of concentration . For example, an increase in psychiatric morbidity was observed employing the General Health Questionnaire, the Clinical Interview Schedule, the Social Stress and Support Interview . The Beck Depression Inventory scores were significantly elevated in $20-30 \%$ of the patients 6 months after starting IFN- $\alpha$ immunotherapy . The degree of severity varied from mild (20\%) to severe (4\%) depressive symptoms. The Zung self-rating depression scale was significantly increased in patients treated with IFN- $\alpha$. We evaluated 30 patients, affected by chronic active hepatitis $\mathrm{C}$, before starting IFN- $\alpha$ treatment and 3 months later. The assessments consisted of the DSM-IV criteria for major depression and the Montgomery Asberg Depression Rating Scale (MADRS). At endpoint, $40.7 \%$ of patients suffered from a full blown major depression, according to the DSM-IV criteria. IFN- $\alpha$ treatment induced a significant increase in the MADRS score from baseline to 3 months later. The MADRS items that were significantly increased at endpoint were: expressed and 
unexpressed sadness; irritability; insomnia; loss of appetite; and asthenia. Cognitive symptoms such as feelings of guilt and shame were thus not significantly influenced by IFN- $\alpha$-based immunotherapy. A percentage (1-2\%) of patients treated with IFN- $\alpha$-based immunotherapy attempted suicide A few cases of manic psychoses have been recorded during treatment with IFN- $\alpha$.

The neuropsychiatric effects are dose (between 3 and $10 \mathrm{MU}$ ) and time dependent and appear usually between the first and third month of immunotherapy. One week after starting IFN- $\alpha$-based immunotherapy no significant changes in depressive symptomatology as measured by means of the MADRS rating scale could be found . The psychiatric symptoms following IFN- $\alpha$-based immunotherapy are thus probably obtained after repeated administration during several weeks. During long-term treatment, psychiatric side-effects not infrequently lead to a reduction or discontinuation of the immunotherapy. Some authors argued that immunotherapy with IFN- $\alpha$ may aggravate the severity of pre-existing symptomatology, such as anxiety, depression, fatigue, and impaired concentration. Patients with a subclinical symptomatology may thus be more predisposed to IFN- $\alpha$-induced mood disturbances. However, IFN- $\alpha$-induced major depression may also occur in a population with no subclinical symptoms and without a history of past psychiatric disorders . It is important to stress that the psychiatric side-effects may be reversible . Case reports have shown that IFN- $\alpha$-induced major depression may be responsive to therapy with antidepressants, e.g. selective serotonin re-uptake inhibitors and tricyclic antidepressants. Patient education is an important promoter of compliance, as are the proper detection and symptomatic treatment of the side-effects

\section{Behavioural effects of interferon- $\alpha$}

Interferons have been tested in experimental animals.

The forced swimming test-induced immobility is regarded as an index of depression and has therefore been used as an animal model of depression. An immobile posture observed during the test is thought to indicate 'behavioural despair'. In mice, human IFN- $\alpha$ injected intravenously at the dose of $6 \times 10^{4} \mathrm{IU} / \mathrm{kg}$ increased the immobility time in the forced swimming test Administration of IFN- $\beta$ and IFN- $\gamma$ did not result in the same behavioural effects. IFN- $\beta$ or IFN- $\gamma$ thus did not increase immobility in the forced swimming test in mice at a dose of $6 \times 10^{4} \mathrm{IU} / \mathrm{kg}$

Interferon- $\alpha$ and the central nervous system Recent evidence indicates that IFN- $\alpha$ may modulate many functions in the central nervous system (CNS). Interferons ( $\alpha$ and $\gamma$ ) can enter the CNS from the periphery in a regional selective manner. The permeability of the spinal cord is higher than that of the brain, and for each region the permeability to IFN- $\alpha$ is greater than that to IFN- $\gamma$. The mechanism involved seems to be an endothelial transcytosis. Yamada and Yamanaka found IFN- $\alpha$ receptors in microglial cells as well as in macrophages in cerebral infarct areas. Invitro experiments showed that astrocytes produced IFN- $\alpha$ when treated with Newcastle disease virus In Sprague-Dawley rats IFN- $\alpha$ modulated the majority of the hypothalamic (70\%), amygdala $(76 \%)$, hippocampus $(75 \%)$ and cortical $(82 \%)$ cells whether the route of administration was within the brain or given peripherally (intravenously or intraperitoneally). IFN- $\alpha$ in general suppressed the hypothalamic neuronal activity while accelerating neuronal acrivity in all the other CNS sites

. The authors concluded that IFN- $\alpha$ is an endogenous peptide synthesized and released both peripherally and centrally, with the same effects on neuronal activity whether it is given systemically or locally within the brain. IFN- $\alpha$ induced the release of corticotropinreleasing factor and arginine vasopressin from neurons in the hypothalamus and the amygdala . The latter is a brain structure with several roles in the stress response and emotional memory, and in the regulation of the hypothalamic-pituitary-adrenal axis. In addition, IFN- $\alpha$ may modulate physiological functions through opioid receptors , and seems to play an antagonistic role with naloxone in binding to membrane sites. The interaction of IFN- $\alpha$ with opioid receptors could explain the attenuation of morphine withdrawal symptoms when IFN- $\alpha$ is injected systemically $1 \mathrm{~h}$ before naloxone Naloxone reduced the IFN- $\alpha$ increased immobility time in the forced swimming test in the mouse?. The above results suggest that IFN- $\alpha$ should be considered to be a neuromodulator.

\section{Interferons and neurotransmitters relevant to the pathophysiology of depression}

Interferons play an important role in the modulation of neurotransmitters, which are involved in the pathophysiology of depression, i.e. catecholamines and serotonin. The treatment of cultured adrenal medullary cells with IFN- $\alpha$ causes a decrease in the uptake of $\left[{ }^{3} \mathrm{H}\right]$ noradrenaline by the cells in a time and concentration-dependent manner. Scatchard analysis of $\left[{ }^{3} \mathrm{H}\right]$ desipramine binding shows that IFN- $\alpha$ decreases the maximal binding $\left(B_{\max }\right)$ values without any change in the dissociation constant $\left(\mathrm{K}_{d}\right)$ values. These findings suggest that IFN- $\alpha$ suppresses the function of the noradrenaline transporter by reducing the density of the transporter in cell membranes . In mice, Shuto et al. assessed the changes in the dopaminergic, serotonergic and adrenergic systems after chronic intraperitoneal administration of IFN- $\alpha$. After cerebral (the cerebellum was excluded) homogenization and detection of the neurotransmitters 
and their metabolites, a decrease in dopaminergic concentration was found but no alterations in the serotonergic or noradrenergic system. The treatment of rats with IFN- $\alpha$ significantly reduced in a dosedependent manner serotonin levels in the frontal cortex, midbrain and striatum . Treatment with IFN- $\alpha$ and IFN- $\gamma$ of human placental choriocarcinoma cells increased both the serotonin transporter messenger RNA and the uptake activity of the serotonin transporter Interferons ( $\alpha, \beta$, and $\gamma$ ), IL-1, IL-2 and lipopolysaccharide may induce indoleamine 2,3-dioxygenase (IDO)

IDO is the major tryptophan catabolizing enzyme in the brain, kidney, lung, spleen, duodenum and immune cells, which induces the catabolism of tryptophan to kynurenine. Although IFN- $\gamma$ directly affects IDO activity, IFN- $\alpha$ has an indirect effect through a $15000 \mathrm{M}_{\mathrm{r}}$ protein, which is a product of IFN- $\alpha$-treated monocytes and lymphocytes and stimulates IDO and IFN- $\gamma$ production . Inflammation and infection may thus elicit proinflammatory cytokines, which stimulate the catabolism of tryptophan by inducing IDO, which in turn inhibits $T$ cell proliferation, owing to reduced access of tryptophan . This mechanism may play an important role in antimicrobial defences and in the protection from attacks by autoreactive $\mathrm{T}$ cells. Finally, IFN- $\alpha$ significantly increases both the $K_{d}$ and $B_{\text {max }}$ measures of $\left[{ }^{3} \mathrm{H}\right] 8-\mathrm{OH}-\mathrm{DPAT}$ binding at low-affinity binding sites, but not at high-affinity sites . These results suggest that IFN- $\alpha$ affects the low-affinity serotonin1A receptor sites.

Interferon- $\alpha$, major depression, serotonin and immune activation

Major depression is accompanied by an activation of the inflammatory response system (IRS) and by serotonergic disturbances. IRS activation is indicated by the following: (i) increased numbers of peripheral blood neutrophils, monocytes and activated T cells; (ii) increased plasma concentrations of positive acute phase reactants, such as haptoglobin and C-reactive protein and decreased plasma concentrations of negative acute phase proteins (APPs) reactants, such as albumin and zinc; (iii) increased production of monocytic and $\mathrm{T}$ lymphocytic products, such as neopterin, prostaglandin E2, and elastase; and (iv) increased production of pro-inflammatory cytokines, such as IL- $1 \beta$, IL-6 and IFN- $\gamma$

IRS activation and administration of pro-inflammatory cytokines, such as interferons, IL-1 and IL-6, may induce depressive and anxiety symptoms through, among other things, their modulation of the central and peripheral serotonergic systems . The specific serotonergic disturbances found in depression are a lowered activity of the central presynaptic serotonin neurons, which is partly related to a lowered availability of plasma tryptophan; and changes in postsynaptic receptors such as increased number, affinity or respon- sivity of postsynaptic serotonin2A/C receptors; and downregulated or desensitized postsynaptic serotonin1A receptors . IFN- $\alpha$-induced changes in serotonergic turnover, the expression of the serotonin transporter, the catabolism of tryptophan and serotonin1A receptor characteristics may thus be involved in the development of IFN- $\alpha$-induced psychiatric disturbances, such as major depression. Recently, we found that immunotherapy with IFN- $\alpha$ significantly induced a significant activation of the cytokine network, i.e. the serum concentrations of IFN- $\gamma$, IL- 6 , IL- 8 and IL-10 were significantly higher 1 or 4 months after starting immunotherapy with IFN- $\alpha$ than at baseline (S. Bonaccorso, V. Marino, A. Puzella, et $a l$, in preparation). Moreover, immunochemotherapy with IFN- $\alpha$ for $1-4$ months induced significant increases in the MADRS scores and significantly increased the plasma concentrations of kynurenine; and induced significant reductions in plasma tryptophan and serotonin concentrations (S. Bonaccorso, V. Marino, A. Puzella, et al., in preparation). Immunotherapy with IFN- $\alpha$ thus significantly stimulates the catabolism of tryptophan, thereby decreasing the availability of tryptophan to the brain. There were significant time-relationships between the IFN- $\alpha$-induced changes in the MADRS and those in the serotonergic markers. Therefore, we suggest that a number of factors could play a role in the development of IFN- $\alpha$-induced major depression, i.e. (i) IFN- $\alpha-$ induced activation of the cytokine network with increased production of pro-inflammatory cytokines, which show depressogenic effects; (ii) stimulation of the catabolism of tryptophan through the induction of IDO; and (iii) depletion of the turnover of serotonin.

\section{References and recommended reading}

\section{Papers of particular inter}

Peen highilighted as:

- of outstanding interest

1 Maddrey WC. Safety of combination interferon alfa-2b/ribavirin therapy in Maddrey WC. Safety of combination interferon alta-2b/ribavirin therapy in
chronic hepatitis C-relapsed treatment-naive patients. Semin Liver Dis 1999; chronic hep
19:67-75.

2 Billiau A. The mode of action of interferons in viral infections and their possible role in the control of hepatitis B.J Hepatol 1986; 3 (Suppl. 2):S171

3 Emstoff MS, Gooding W, Nair S, et al. Immunological effects of treatment with sequential administration of recombinant interferon gamma and alpha in patients with metastatic renal cell carcinoma during a phase I trial.
Res 1992; 52:851-856.

4 Numa $Y$, Kawamoto $K$, Sakai N, Matsumura H. Flow cytometric analysis of antineoplastic effects of interferon-alpha, beta and gamma with fluorescein isothiocyanate on cultured brain tumors. J Neurooncol 1991; 11:225-234.

5 Finter NB, Chapman S, Dowd P, et al. The use of interferon-alpha in virus infections. Drugs $1991 ; 42: 749-765$.

6 Inglot $A D$, Leszek J, Piasecki $E$, Sypula $A$. Interferon responses in schizophrenia and major depressive disorders. Biol Psychiatry 1994; 35:464-473.

7 Dusheiko G. Side effects of alpha interferon on chronic hepatitis. C. Dusheiko G. Side effects of alpha interferon
Hepatology 1997; 26 (3 Suppl. 1):112S-121S. 8 Vial $T$, Descotes J. Clinical toxicity of the interferons. Drug Safety 1994;
10:115-150. 
9 Pavol MA, Meyers CA, Rexer IL, et al. Pattern of neurobehavioral deficits associated with interferon alfa therapy for leukemia. Neurology 1995;

10 Mattson $K$, Nirranen A, Laaksonen $R$, Cantell K. Psychometric monitoring of
interferon neurotoxicity. Lancet 1984; 1:275-276.

11 Renault PF, Hoofnagle JH, Park Y, et al. Psychiatric complications of longterm interferon alfa therapy. Arch Intern Med 1987; 147:1577-1580.

12 Adams F, Quesada JR, Gutterman JU. Neuropsychiatric manifestations of human leukocyte interferon therapy in patients with cancer. JAMA 1984

13 Hardy P. Trouble depressifs et interferon alpha. Gastroenterol Clin Biol 1996; 20:255-257. 14 Valentine AD, Meyers CA, King MA, et al. Mood and cognitive side effects of
interferon-alpha therapy. Semin Oncol 1998; 25:39-47.

15 Heeringa $M$, Honkoop $P$, de Man RA, et al. Major psychiatric side effects of Heeringa M, Honkoop P, de Man RA, et al. Major psychiatric side eft
interferon alpha-2b. Ned Tijdschr Geneesk 1998; 142:1618-1621.

16 McDonald EM, Mann AH, Thomas HC. Interferons as mediators of psychiatric morbidity. An investigation in a trial of recombinant alpha-

17 Hunt C, Dominitz JA, Bute BP, et al. Effect of interferon-alpha treatment of
chronic hepatitis C on health-related quality of life. Dig Dis Sci 1997; chronic hepatitis

18 Malaguarnera $\mathrm{M}$, Di Fazio $\mathrm{L}$, Restuccia $\mathrm{S}$, et al. Interferon alpha-induced depression in chronic hepatitis $C$ patients: comparison betwe
types of interferon alpha. Neuropsychobiology 1998; 37:93-97.

19 Bonaccorso S, Marino V, Biondi M, et al. Major depression induced by interferon-alpha in patients affected by hepatitis C virus. J Affect Disord 2000; (in press).

This study examines 30 patients, affected by chronic active hepatitis C, both
before and after immunotherapy with IFN- $\alpha$. The evaluation consisted of psychometric assessments employing the DSM-IV criteria and the MADRS. After 3 months immunotherapy, $40.7 \%$ of the patients suffered from a full blown major depression. FN- $\alpha$ treatron induced a signicant increase in the MADRS scor and the vegetative, but not cognitive, symptoms of depression.

20 Janssen HL, Brouwer $\pi$, van der Mast RC, Schalm SW. Suicide associated with alfa-interferon therapy for chronic viral hepatitis. I Hepatol 1994; 21:241-243.

21 Fattovich G, Giustina G, Favarato S, Ruol A (Investigators of the thalian patients with chronic viral hepatitis treated with alpha interferon. I Hepato patients with chro
1996; 24:38-47.

22 Rifflet $H$, Vuillemin $E$, Oberti $F$, et al. Interferon et suicide au cours des hepatites virgules chroniques. Gastroenterol Clin Biol 1996; 20:606-607.

23 Windemuth D, Bacharach-Buhles $M$, Hoffmann $K$, Altmeyer P. Depression and suicidal intentions as a side effect of high dosage interferon alpha

24 Kanno A, Yamada M, Abe M, Okamoto Y. A case of interferon alpha-induced
manic psychosis in chronic hepatitis. Tohoku J Exp Med 1999; 187:79-82.

Pand A. Prediction of the depressive effects of intereron alf therapy by the patient's initial affective state. N Engl I Med 1999; 340:13-70.

26 Capuron L, Ravaud A, Dantzer R. Early depressive symptoms in cancer patients receiving interleukin 2 and/or interferon alfa therapy. J Clin Oncol 2000;

This study shows that patients treated with IL-2 alone or in association with IFN- $\alpha$
had significantly higher MADRS scores after 5 days of cytokine therapy, and patients who received both cytokines had increased scores on day 3 . In contrast patients treated with IFN- $\alpha$ alone had varying MADRS scores during the course of treatment. It was concluded that IL-2 and IFN- $\alpha$ have differential effects on mood, and that IL-2 immunotherapy induces depressive symptoms early in treatment, whereas the psychiatric effects of $\mathrm{F} N-\alpha$ occur later in treatment.

27 Miyaoka $H$, Otsubo $T$, Kamijima K. Depression from interferon therapy in patients with hepatitis C. Am J Psychiatry 1999; 156:11-20. 28 Rosenstein DL, Lerner D, Cai J. More on the depressive effects of interferon
alfa. N Engl J Med 1999; 341:849-850.

29 Okanoue $T$, Sakamoto $S$, ttoh $Y$, et al. Side effects of high-dose interferon therapy for chronic hepatitis C. J Hepatol 1996; 25:283-291.

30 Gleason OC, Yates WR. Five cases of interferon-alpha-induced depression treated with antidepressant therapy. Psychosomatics 1999; 40:510-512.

31 Schafer $M$, Messer $T$, Wegner $U$, et al. Psychiatric side effects during adjuvant therapy with interferon-alpha in patients with malignant melanoma. 1999; 50:654-658.
32 Meyers CA. Mood and cognitive disorders in cancer patients receiving cytokine therapy. Adv Exp Med Biol 1999; 461:75-81.

This paper reviews the theory that chronic treatment with cytokines, including IFN$a$ is associated with the development of mood and cognitive changes that uggests frontalsubcortical cerebral dysfunction.

33 Berk $L$, van Gool AR, Bannink M, Kruit WH. Side effects of interferon alfa. Ned Tijdschr Geneeskd 1999; 143:1461-1464.

34 Valentine $A D$, Meyers CA. Successful treatment of interferon-alpha-induce mood disorder with nortiptyline. Psychosomatics 1995; 36:418-419.

35 Goldman LS. Successful treatment of interferon alfa-induced mood disorder with nortriptyline. Psychosomatics 1994; 35:412-413.

36 Levenson JL, Fallon HJ. Fluoxetine treatment of depression caused by
interferon-alpha. Am J Gastroenterol 1993; 88:760-761.

7 Makino $M$, Kitano $Y$, Komiyama $C$, et al. Human interferon-alpha induces immobility in the mouse forced swimming test: involvement of the opioid
system. Brain Res 2000; 852:482-484.

38 Pan W, Banks WA, Kastin AJ. Permeability of blood-brain and blood-spinal cord barriers to interferons. J Neuroimmunol 1997; 76:105-111.

39 Yamada $T$, Yamanaka I. Microglial localization of alfa-interferon receptor in Y Y The

40 Lieberman A, Pitha PM, Shin HS, Shin ML. Production of tumor necrosis tactor and other cytokines by astrocytes stimulated with lipopolysacch.
a neurotropic virus. Proc Natl Acad Sci USA 1989; 86:6348-6352.

41 Dafny N, Prieto-Gomez B, Dong W-O, Reyes-Vazquez C. Interferon modulates neuronal activity recorded from the hypothalamus, thalamus, hippocampus, amygdala and the somatosensory cortex. Brain Res 1996
734:269-274.

42 Raber J, Koob GF, Bloom FE. Interferon alfa and transforming growth factorbeta-1 regulate corticotropin-releasing factor release from the amygdala: 463.

43 Menzies RA, Patel R, Hall NRS, et al. Human recombinant interferon alpha inhibits naloxone binding to rat brain membranes. Life Sci 1992; 50:227-232

44 Dafny N, Dougherty PM, Lee JR. Immune response products alter CNS activity: interferon modulates central opioid functions. J Neurosci Res 1988 19:130-139.

45 Toyohira $\mathrm{Y}$, Yanagihara $\mathrm{N}$, Minami $\mathrm{K}$, et al. Down-regulation of the noradrenaline transporter by interferon-alpha in cultured bovine adrenal
medullary cells. J Neurochem 1998; 70:1441-1447.

46 Shuto $H$, Kataoka $Y$, Horikawa $T$, et al. Repeated interferon-alpha administration inhibits dopaminergic neural activity in the mouse brain. Brain Res 1997; 747:348-35

47 Kamata $M$, Higuchi $H$, Yoshimoto $M$, et al. Effect of single intracerebroventricular injection of alpha-interferon on monoamine concentration in the rat

48 Morikawa $O$, Sakai $N$, Obara $H_{\text {, Saito }} N$. Effects of interferon-alpha, interferon-gamma and CAMP on the transcriptional regulation of the seroton transporter. Eur J Phammacol 1958 , $349 \cdot 317$

49 Taylor MW, Feng G. Relationship between interferon-gamma, indolamine

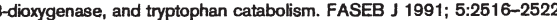

50 Recht $M$, Borden EC, Knight E. A human 15-kDa IFN-induced protei induces the secretion of IFN-gamma. J Immunol 1991; 147:2617-2623.

51 Mellor AL, Munn DH. Tryptophan catabolism and T-cell tolerance: immunosuppression by starvation? Immunol Today 1999; 20:469-473.

52 Abe S, Hori T, Suzuki T, et al. Effects of chronic administration of interferon alpha ADD on serotonergic receptors in rat brain. Neurochem Res 1999; 24:359-363.

This important study examines the effects of the chronic administration of IFN- $\alpha$ on serotonergic binding sites in the rat brain. IFN- $\alpha$ was injected daily for 2 weeks at a dose of $100000 \mathrm{IU} / \mathrm{kg}$ (intraperitoneally) in male Wistar rats. IFN- $\alpha$ significantly increased both $\mathrm{K}_{d}$ and $\mathrm{B}_{\max }$ measures of $\left.{ }^{3} \mathrm{H}\right] \mathrm{B}-\mathrm{OH}-\mathrm{DPAT}$ binding at low-affinity binding sites, but not at high-aftinity sites. These results suggest that IFN- $\alpha$ affects the low-affinity serotonin1A receptor sites and may be involved in the development
of IFN-induced psychiatric disturbances.

53 Maes M, Smith R, Scharpe S. The monocyte T lymphocyte hypothesis of major depression. Psychoneuroendocrinology 1995; 20:111-116. 
54 Maes M. Major depression and activation of the inflammatory response system. Adv Exp Med Biol 1999; 461:25-46.

This paper reviews the cytokine hypothesis of depression showing that endogenous major depression is accompanied by activation of the inflammatory tesponse system, that organic depressions may be induced by intarn ( network.
55 Yirmiya R. Behavioral and psychological effect of immune activation: implication for depression due a general medical condition. Curr Opin 56 Maes $M$, Meltzer HYM. The serotonin hypothesis of major depression. In:
Psychopharmacology the fourth generation of progress. Bloom FE, Kupfer $D$. Psychopharmacology the fourth generation of progress. 
44 Cytokines and Depression: A Neurochemical Hypothesis 


\title{
Chapter 5
}

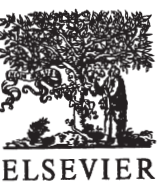

PSYCHIATRY

RESEARCH

\section{Immunotherapy with interferon-alpha in patients affected by chronic hepatitis $\mathrm{C}$ induces an intercorrelated stimulation of the cytokine network and an increase in depressive and anxiety symptoms}

\author{
Stefania Bonaccorso a,b,, Antonella Puzella ${ }^{a}$, Valentina Marino ${ }^{a}$, \\ Massimo Pasquini ${ }^{\mathrm{a}}$, Massimo Biondi ${ }^{\mathrm{a}}$, Marco Artini ${ }^{\mathrm{d}}$, \\ Cristiana Almerighi ${ }^{\mathrm{d}}$, Massimo Levrero ${ }^{\mathrm{d}}$, Belinda Egyed ${ }^{\mathrm{b}}$, \\ Eugene Bosmans ${ }^{\mathrm{e}}$, Herbert Y. Meltzer ${ }^{\mathrm{c}}$, Michael Maes ${ }^{\mathrm{b}, \mathrm{c}, *, 1}$ \\ ${ }^{a}$ Psychiatric Hospital, University 'La Sapienza', Rome, Italy \\ ${ }^{b}$ Department of Psychiatry, University Hospital of Maastricht, Maastricht, The Netherlands \\ ${ }^{c}$ Department of Psychiatry, Vanderbilt University, Nashville, TN, USA \\ ${ }^{\mathrm{d}}$ Department of Hepatology, I Medical Clinic, University 'La Sapienza', Rome, Italy \\ ${ }^{e}$ Eurogenetics, Tessenderlo, Belgium
}

Received 30 January 2001; received in revised form 18 September 2001; accepted 20 September 2001

\section{Abstract}

Immunotherapy with interferon-alpha (IFN $\alpha$ ) may induce depressive symptoms, anxiety and major depression when administered for at least 1-3 months at a dose of 3-10 MUI daily, twice or three times a week. Previously, it has been shown that immunotherapy with interleukin-2 (IL-2) significantly induces the cytokine network, as measured by increases in serum IL-6, IL-10 and the IL-2 receptor (IL-2R), and that the immunotherapy-induced changes in the cytokine network are significantly correlated with the increases in depression ratings. The main aim of this study was to examine the effects of immunotherapy with IFN $\alpha$ on the cytokine network in relation to changes in depression and anxiety ratings. Fourteen patients, affected by chronic active C-hepatitis, were treated with IFN $\alpha$ (3-6 MUI s.c. three/six times a week for 6 months) and had measurements of serum IFN-gamma (IFN $\gamma$ ), IL-2, IL-6, IL-6R, IL- 8 and IL-10 before starting therapy and 2, 4, 16 and 24 weeks after immunotherapy with IFN $\alpha$. Severity of

\footnotetext{
* Corresponding author. Department of Psychiatry \& Neuropsychology, University Hospital of Maastricht, Postbus 5800, 6202 AZ Maastricht, The Netherlands. Tel.: +31-43-3877443; fax: +31-43-3875444.

E-mail address: crc-mh@online.be (M. Maes).

${ }^{1}$ http://www.marquiswhoswho.net/mmaes/

0165-1781/01/\$ - see front matter @ 2001 Elsevier Science Ireland Ltd. All rights reserved.

PII: S $0165-1781(01) 00315-8$
} 
depression and anxiety were measured with the Montgomery-Åsberg Depression Rating Scale (MADRS) and the Hamilton Anxiety Rating Scale (HAM-A), respectively. Repeated measure (RM) design ANOVAs showed significantly higher MADRS and HAM-A scores 2-4 weeks and 4-6 months after starting IFN $\alpha$-based immunotherapy than at baseline. RM design ANOVAs showed significantly higher serum IL-6 and IL-8 levels 2-4 weeks after starting IFN $\alpha$-based immunotherapy and higher serum IL-10 levels 2-4 weeks and 4-6 months after starting therapy than at baseline. There were significant relationships between the IFN $\alpha$-induced changes in serum IL-6 or IL-8 and the depression and anxiety scores. The findings show that IFN $\alpha$-based immunotherapy induces the cytokine network and that IFN $\alpha$-induced increases in IL-6 predicts the development of depressive symptoms. Depressive symptoms following IFN $\alpha$ treatment may be secondary to cytokine induction, including that of IL-6. (c) 2001 Elsevier Science Ireland Ltd. All rights reserved.

Keywords: Interferon-alpha; Immunotherapy; Cytokines; Depression; Anxiety; Interleukin-6; Hepatitis C

\section{Introduction}

Hepatitis $\mathrm{C}$ virus (HCV) chronic infection may be associated with an activation of the host cellular immune defense. Recent evidence points towards an imbalance between the two different populations of T-helper (Th) cells (Th-1 and Th-2) in its pathogenesis. Patients affected by chronic hepatitis show increased serum levels of the IL-2 receptor (IL-2R), which is a T-cell activation indicator, IL-6 (a pro-inflammatory cytokine), and neopterin, a product of the biopterin pathway secreted by macrophages after induction by interferon-gamma (IFN $\gamma$ ) (Grungreiff et al., 1999; Naveau et al., 1999). The hypothesis of a Th1-Th2 imbalance is confirmed by a report showing an increase in mRNA expression of Th1-type cytokines, such as IL-2 and IFN $\gamma$, and a down-regulation of IL-4 and IL-10 mRNA, both Th-2-type cytokines, in the chronic evolution of the disease (Tsai et al., 1997).

Immunotherapy with IFN $\alpha$ is the treatment of choice for patients with chronic hepatitis-C (Maddrey, 1999). IFN $\alpha$ is a cytokine produced by lymphocytes, null lymphocytes and macrophages. It has pleiotropic functions which include potent antiviral and antiproliferative effects. IFN $\alpha$ acts directly on the cells infected by the virus and indirectly via the cytokine network (Malaguarnera et al., 1997). Immunotherapy with IFN $\alpha$ may induce or inhibit the expression of several other cytokines, as well as cytokine receptors, such as interleukin-1 (IL-1), IL-2, IL-6, IL-8, IL-1 receptor, IL-1 receptor antagonist, tumor necrosis factor (TNF) and IFN $\gamma$ (Taylor and Grossberg, 1998;
Grungreiff et al., 1999; Kawamura et al., 1999; Malaguarnera et al., 1997; Shimizu et al., 1995; Cramp et al., 2000). The mechanism by which IFN $\alpha$ induces expression of these cytokines is not clear. There is some evidence that IFN $\alpha$ treatment may elicit a Th-1-type response. This immune response might help the organism in clearing the virus-infected hepatocytes, and once $\mathrm{HCV}$ is completely eliminated and Th- 1 cells not activated any longer, it may allow the $\mathrm{Th}-2$ system to reach higher levels and ameliorate the tissuedamaging effects of the immune response (Cacciarelli et al., 1996).

There is now evidence for a high incidence rate of neuro-psychiatric complications during longterm therapy with IFN $\alpha$ (review: Bonaccorso et al., 2000, 2001). The symptoms that are induced by IFN $\alpha$-based immunotherapy are: slowness, severe fatigue, hypersomnia, lethargy, depressed mood, mnemonic troubles, irritability, short temper, emotional lability, social withdrawal, and lack of concentration. We found that prolonged IFN $\alpha$ treatment may induce major depression in a considerable number of subjects (i.e. 40.7\%) (Bonaccorso et al., 2001). Moreover, major depression is reported in high frequency as a reason for dose reduction or for stopping immunotherapy (review: Bonaccorso et al., 2000, 2001). We have hypothesized that some of these adverse effects associated with IFN $\alpha$-based immunotherapy may be caused by the induction of other cytokines and their respective behavioral effects. Thus, side effects such as anorexia, fatigue, depressed mood and loss of interest can be caused by one or more 
of the cytokines induced by IFN $\alpha$ (Bonaccorso et al., 2000; Maes et al., 2001). Administration of endotoxin to rats decreases the free consumption of saccharin, a model of anhedonia (Yirmiya, 1996). It is now well established that administration of IL-1 to experimental animals may produce behavioral alterations and symptoms similar to those observed in major depression, such as anhedonia, anorexia, weight loss, social withdrawal, psychomotor retardation, inergia, irritability and sleep disturbances (Yirmiya, 1996; Bluthé et al., 1992; Maier and Watkins, 1998; Anisman et al., 1998; Linthorst and Reul, 1998). Sustained elevated serum levels of IL- 6 in mice result in a rapid decline in preference for sucrose (Sakic et al., 1997). Administration of IL- $1 \beta$ and TNFa induces 'anxiogenic-like' effects on the elevated plus maze (Connor et al., 1998). We found that IL-2-based immunotherapy (at a dose of 18 MUI or subcutaneous $18 \mathrm{MUI} / \mathrm{m}^{2}$ per day during 5 consecutive days) significantly increases serum IL-6 and IL-2R concentrations in parallel with increases in severity of depression ratings (Capuron et al., submitted; Maes et al., 2001). Therefore, we hypothesize that IFN $\alpha$ treatment in hepatitis $\mathrm{C}$ patients may induce the cytokine network, as measured by increased serum concentrations of IL-2, IL-6, IL-8, IL-10 and IFN $\gamma$, and that the induction of the cytokine network and, thus, the increases in the above cytokine levels are correlated to the occurrence of depressive symptoms. Hence, the aims of the present study are to examine whether therapy with IFN $\alpha$ in patients with hepatitis $\mathrm{C}$ induces the cytokine network, as measured by means of the serum concentrations of IL-2, IL-6, IL-8, IL-10 and IFN $\gamma$; and whether the immunotherapy-induced activation of the cytokine network and, in particular, an increase in serum IL-6 are related to IFN $\alpha$-induced depressive or anxiety symptomatology.

\section{Methods}

\subsection{Subjects}

Eighteen patients (10 males and 8 women; mean age \pm S.D. $=40.5 \pm 11.5$ years) affected by chronic active C-hepatitis have been consecutively recruited at the Hepathology Department, I Medical Clinic at the University of Rome 'La Sapienza', between November 1997 and May 1998. Four of the 18 patients suspended IFN $\alpha$ therapy soon after starting treatment; one due to a reduction in the number of platelets, two because of low compliance and one because of a concomitant thyroiditis. Fourteen patients ( 8 males and 6 women; mean age \pm S.D. $=41.7 \pm 10.8$ years) completed the IFN $\alpha$ treatment, the psychiatric evaluations and the blood collections for assay of the serum cytokines.

The diagnosis of chronic active C-hepatitis was based on persistently elevated transaminase values (1.5 over the normal range for at least 6 months), anti-HCV positivity and histological report. Inclusion criteria were: age $<65$ years; histological diagnosis of chronic hepatitis; positivity for anti-HCV antibodies and HCV RNA in serum; and platelet counts $>100.000 / \mathrm{mm}^{3}$. Exclusionary criteria for patients were: liver cirrhosis, heart, kidney or autoimmune diseases, infection with HBV, HDV or HIV, WBC counts $<4000 / \mathrm{mm}^{3}$, $\mathrm{PT}<60 \%$, chronic alcohol abuse or active intravenous drug use (IVDU). We excluded patients with a past or present history of axis-I disorders, such as major and bipolar depression. Illness onset and awareness ranged from a minimum of 1 to a maximum of 2 years. Before starting IFN $\alpha$-based immunotherapy, none of the patients were taking psychotropic drugs, such as antidepressants, antipsychotics or benzodiazepines. Moreover, patients did not take any other medication beside IFNa during the study period. No changes in the physical status were recorded during the treatment period. The local ethical committee approved the study design and the subjects gave written informed consent after the study protocol was fully explained.

We also examined seven normal controls to compare the serum concentrations of IL-6, IL-6R, IL-8 and IL-10 between hepatitis-C patients and the normal control values. Normal controls were free of all medical drugs for at least 1 month prior to blood sampling. No one was a regular drinker or had ever taken psychotropic or im- 
munomodulating drugs. Controls were excluded for a present, past or family history (first-degree relatives) of psychiatric disorders; any medical illnesses; the presence of acute infectious, allergic or inflammatory responses for at least 2 weeks prior to the study; abnormal physical examination and abnormal blood and urine tests, such as SGOT, SGPT, $\gamma \mathrm{GT}$, serum electrolytes, thyroid function tests and renal tests (blood urea and creatinine).

\subsection{Methods}

Patients eligible for IFN $\alpha$ treatment were randomized to receive one of two treatment schedules with IFN $\alpha 2$ a (Roferon, Roche): schedule A $(n=10), 6$ MUI IFN $\alpha$ s.c. per day, 6 days a week for 4 weeks; schedule B $(n=4), 6$ MUI IFN s.c. t.i.w. for 4 weeks. Since there were no significant differences in any of the clinical ratings and biological variables between the two treatment groups at any time point, we performed further statistical analyses on both treatment groups combined. Both groups continued therapy with 6 MUI IFN $\alpha$ s.c. t.i.w. for 12 weeks. Responders at 4 months received 3 MUI IFN s.c. t.i.w. for a further 8 months. Overnight fasting serum for the assay of cytokines was always collected at $08.00 \mathrm{~h}$ at baseline (before starting treatment) and 2, 4, 16 and 24 weeks after starting IFN $\alpha$-based immunotherapy. During each of these sessions, patients completed the Montgomery-Åsberg Depression Rating Scale (MADRS; Montgomery and Asberg, 1979) and the Hamilton Anxiety Rating Scale (HAM-A; Hamilton, 1959). Serum IL-6, IL2 , IL-8, IL-10, IFN $\gamma$ and IL-6R were assayed by means of ELISAs based on appropriate and validated sets of monoclonal antibodies. All serum specimens for the above immune markers were assayed in a single run with a single lot number of reagents and consumables employed by a single operator. The intra-assay coefficients of variation for all immune variables were $<8.0 \%$. The operators were blind to the treatment schedules of the patients.

\subsection{Statistics}

Relationships between variables were checked by means of Pearson's Product-moment correlation coefficients. Time relationships were computed by means of regression analyses, which were pooled over the repeated measurements in the patients. This method eliminates inter-individual variability and assesses the relationships over time between the variables introduced in the regression analysis. Group mean differences were assessed by means of analysis of variance (ANOVA). To check the effects of IFN $\alpha$-based immunotherapy on the immune and clinical measurements, we employed repeated measure (RM) design ANOVAs, which were performed on the baseline values and the values obtained during IFN $\alpha$ treatment. Toward this end, we used an RM design ANOVA Mixed Model to check the overall effects of IFN $\alpha$. Since there were no significant differences in any of the variables either between weeks 2 and 4 or between months 4 and 6 and since we wanted to check the early (first month) vs. protracted (several months) effects of IFN $\alpha$-based immunotherapy, we used the peak values of the serum levels obtained at 2 and 4 weeks and of those obtained at 4 and 6 months during IFN $\alpha$-based immunotherapy. Consequently, RM design ANOVAs were performed on the pretreatment values and the peak values obtained 2-4 weeks and 4-6 months after starting IFN $\alpha$-based immunotherapy. All results of RM design ANOVAs were corrected for sphericity. Analyses on simple effects were used to examine the effects of time in different groups. Fisher's least significant difference (LSD) was used to assess the differences between the pre- and posttreatment values.

\section{Results}

Table 1 shows the mean values of the clinical and immune data before and during IFN $\alpha$ treatment. RM design ANOVA Mixed Model showed a significant effect of time on the MADRS ( $F=$ 3.2 , d.f. $=3 / 38, P=0.05)$ and the HAM-A $(F=$ 6.7 , d.f. $=3 / 35, P=0.001)$. RM design ANOVA Mixed Model showed that there were significant effects of time on IL-6 $(F=4.4$, d.f. $=3 / 34, P=$ $0.01)$, IL-8 $(F=4.2$, d.f. $=3 / 34, P=0.03)$ and 
IL-10 $(F=10.6$; d.f. $=3 / 35, P=0.0001)$, but not on plasma IL-2, IFN $\gamma$ or IL-6R. Table 1 also shows the values for IL- 6, IL-8, IL-10 and IL-6R in normal volunteers. There were no significant differences in age (mean $=40.5 \pm 11.5$ years in controls vs. $41.7 \pm 10.8$ years in patients; $F=0.06$, d.f. $=1 / 19, P=0.8$ ) or gender (male/female ratio: $4 / 3$ in controls vs. $8 / 6$ in patients) between the normal volunteers and the hepatitis- $C$ patients. Hepatitis-C patients had significantly higher serum IL-6 $(F=4.8$, d.f. $=1 / 19, P=$ $0.038)$ and IL-10 $(F=19.6$, d.f. $=1 / 16, P=$ $0.0005)$, but lower serum IL-8 $(F=5.1$, d.f. $=$ $1 / 16, P=0.03)$, than normal volunteers. No significant differences in serum sIL-6R could be found between the two study groups $(F=2.4$, d.f. $=1 / 19, P=0.1$ ).

Fig. 1 shows the MADRS and HAM-A scores at the three time points. RM design ANOVAs showed significant effects of time on the MADRS $(F=4.2$, d.f. $=2 / 26, P=0.02)$ and HAM-A $(F=$ 3.7 , d.f. $=2 / 23, P=0.03$ ) scores. LSD showed significantly higher MADRS and HAM-A scores 2-4 weeks and 4-6 months after starting treatment than at baseline.

Fig. 2 shows the measurements of serum IL-6, IL-6R, IL-8, IL-10, IL-2 and IFN $\gamma$ before and during treatment with IFN $\alpha$. RM design ANOVAs showed significant effects of time on serum IL-6, IL-8, and IL-10. LSD showed significantly higher serum IL-6 and IL-8 concentrations 2-4 weeks after starting treatment than at base- line. There was a trend toward significantly higher concentrations of IL-6 and IL-8 in serum 4-6 months after starting immunotherapy than at baseline. RM design ANOVA, followed by LSD testing, showed that serum IL-10 was significantly higher 2-4 weeks and 4-6 months after starting IFN $\alpha$-based immunotherapy than at baseline.

Regression analyses pooled over the three repeated measurements in the subjects did not show any significant correlations between the IFN $\alpha$-induced changes in the MADRS and those in serum IL-6 $(r=0.17, P=0.6)$, IL-8 $(r=0.34, P=0.07)$, IL-6R $(r=0.16, P=0.6)$, IL-10 $(r=0.23, P=0.2)$, IFN $\alpha(r=0.15, P=0.5)$ and IL-2 $(r=-0.01$, $P=0.9$ ). Regression analyses pooled over the three repeated measurements in the subjects did not show any significant correlations between the IFN $\alpha$-induced changes in the HAM-A and those in serum IL-6 $(r=0.18, P=0.6), \mathrm{IL}-8(r=0.31$, $P=0.09)$, IL-6R $(r=0.04, P=0.8), \mathrm{IL}-10(r=$ $0.08, P=0.7)$, IFN $\gamma(r=0.05, P=0.8)$ and IL-2 $(r=0.07, P=0.7)$. This uncoupling of the severity of depression and anxiety and the immune markers may be explained by the findings that the serum concentrations of IL- 6 and IL-8 peaked 2-4 weeks after starting treatment, whereas the MADRS and HAM-A scores remained high throughout the treatment period (see Figs. 1 and 2). Consequently, we have recomputed the relationships between the changes in the immune markers from baseline to 2-4 weeks later and the changes in the MADRS and HAM-A scores from

Table 1

Measurements of the Montgomery-Åsberg Depression Rating Scale (MADRS) and the Hamilton Anxiety Rating Scale (HAM-A), and the serum concentrations of interleukin-6 (IL-6), IL-2, IL-8, IL-10, soluble IL-6 receptor (sIL-6R) and interferon- $\gamma$ (IFN $\gamma$ ) in hepatitis-C patients before (basal) and during (i.e. at 2, 4, 16 and 24 weeks) immunotherapy with IFN

\begin{tabular}{llccccc}
\hline Variables & NC & Basal & 2 weeks & 4 weeks & 16 weeks & 24 weeks \\
\hline MADRS & - & $4.7(3.2)$ & $9.2(9.8)$ & $8.2(8.3)$ & $8.4(7.9)$ & $9.2(6.9)$ \\
HAM-A & - & $6.0(5.0)$ & $9.8(7.1)$ & $4.7(4.8)$ & $9.1(7.1)$ & $7.7(4.5)$ \\
IL-6 pg/ml & $0.9(1.6)$ & $3.5(2.9)$ & $2.9(2.0)$ & $4.9(2.9)$ & $3.7(2.6)$ & $3.6(2.8)$ \\
IL-2 pg/ml & - & $51(94)$ & $44(81)$ & $29(55)$ & $25(46)$ & $75(13)$ \\
IL-8 pg/ml & $39.5(29.6)$ & $17.9(14.7)$ & $23.7(13.2)$ & $23.2(11.4)$ & $20.3(11.3)$ & $16.5(9.7)$ \\
IL-10 pg/ml & $0.9(0.8)$ & $5.9(2.9)$ & $4.1(2.9)$ & $9.4(5.4)$ & $8.5(3.6)$ & $9.0(4.5)$ \\
IL-6R pg/ml & $143(50)$ & $181(54)$ & $216(124)$ & $186(63)$ & $222(122)$ & $173(44)$ \\
IFN IU /ml & - & $0.3(0.9)$ & $0.3(0.9)$ & $0.6(1.3)$ & $0.3(0.6)$ & $1.5(2.9)$ \\
\hline
\end{tabular}

All values are shown as mean (S.D.). Serum IL-6, IL-8, IL-10 and IL-6R were also measured in seven normal controls (NC) to compare the baseline values in hepatitis-C patients with the normal values. 

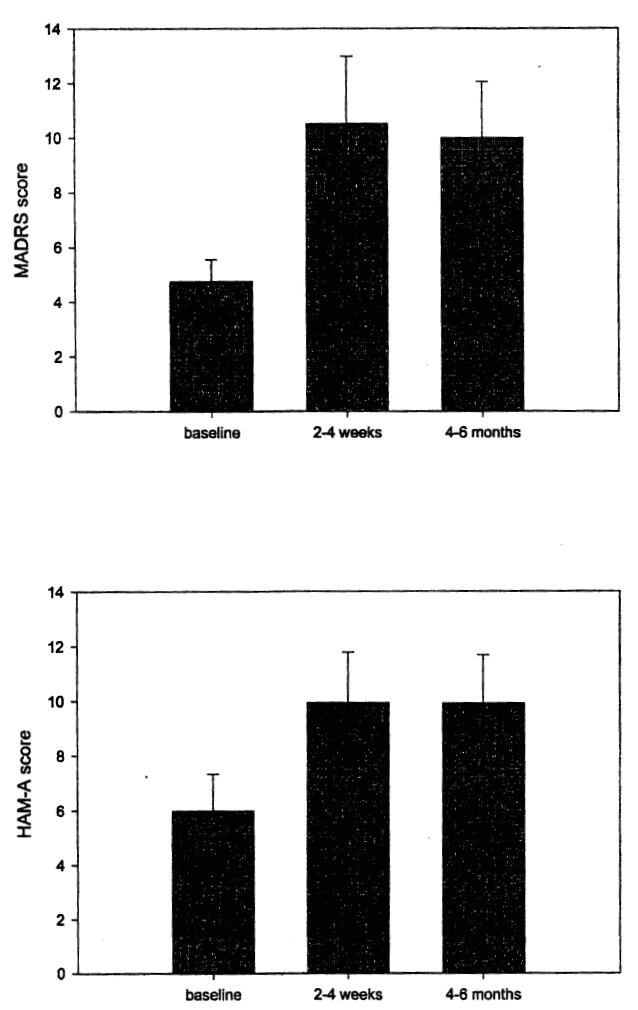

Fig. 1. Effects of interferon-alpha immunotherapy on the Montgomery-Åsberg Depression Rating Scale (MADRS) and the Hamilton Anxiety Rating Scale (HAM-A) in 14 patients with hepatitis $C$, in baseline conditions (basal), and 2-4 weeks and 4-6 months after starting immunotherapy. The results of RM design ANOVAs are described in Section 3.

baseline to 4-6 months later. Regression analyses pooled over these repeated measurements (and applied to the immune variables with significant changes over time in the RM design ANOVAs) showed significant time relationships between the changes in the MADRS score and those in serum IL-6 $(r=0.54, P=0.03)$ and trends toward significant relationships between the changes in the MADRS score and those in serum IL-6R ( $r=$ $0.48, P=0.06)$ and IL-8 $(r=0.45, P=0.09)$. Regression analyses showed significant time rela- tionships between the changes in the HAM-A score and those in serum IL-6 $(r=0.53, P=0.04)$ and IL-8 $(r=0.51, P=0.05)$.

Based on the above correlations between the IFN $\alpha$-induced changes in serum IL-6 at 2-4 weeks and the MADRS score at 4-6 months, we also examined the effects of time on the MADRS score from baseline to 4-6 months later in patients who had a greater IFN $\alpha$-induced IL-6 response 2-4 weeks after starting IFN $\alpha$ treatment ( $n=7$; labeled IL-6 responders) than in those with a lower IFN $\alpha$-induced IL- 6 response ( $n=7$; labeled IL-6 non-responders). IL-6 responders were defined as patients who have IL-6 values that are greater than the median of the residualized IL- 6 values obtained after regression of the peak IL-6 values at 2-4 weeks on the baseline IL-6 values. RM design ANOVA showed a significant effect of time $(F=10.3$, d.f. $=1 / 12$, $P=0.006)$. Analyses on simple effects showed a significant effect of time in IL-6 responders $(F=$ 13.4 , d.f. $=1 / 12, P=0.003$ ), i.e. the MADRS score increased from baseline $(4.0 \pm 3.3)$ to $4-6$ months later (15.0 \pm 5.9$)$. In IL-6 non-responders no significant effects were detected $(F=0.9$, d.f. $=1 / 12, P=0.6$; the MADRS score at baseline was $5.4 \pm 3.2$ and $4-6$ months later $8.3 \pm 7.3$. Analyses of simple effects also showed significantly higher MADRS values in IL-6 responders than in non-responders 4-6 months after starting treatment $(F=5.8$, d.f. $=1 / 24, P=0.02)$, but not in baseline conditions $(F=0.3$, d.f. $=1 / 24, P=$ $0.6)$. These findings show that a higher IFN $\alpha$-induced IL-6 response at 2-4 weeks predicts a higher MADRS score 4-6 months later. It would have been of interest to examine the cytokine responses in subjects who developed major depression during treatment vs. those who did not. Although we previously reported that IFN $\alpha$ may induce major depression in $40 \%$ of patients treated with IFN $\alpha$ (Bonaccorso et al., 2001), the incidence of post-IFN $\alpha$ depression found in the present study $(28.6 \%)$ was too low to perform statistical tests. Therefore, we have examined the differences in serum IL- 6 and IL- 8 between subjects who had a MADRS score $\geq 14$ during treatment $(n=7)$ and those with MADRS scores $<14$ $(n=7)$. RM design ANOVAs showed that serum 
S. Bonaccorso et al. / Psychiatry Research 105 (2001) 45-55
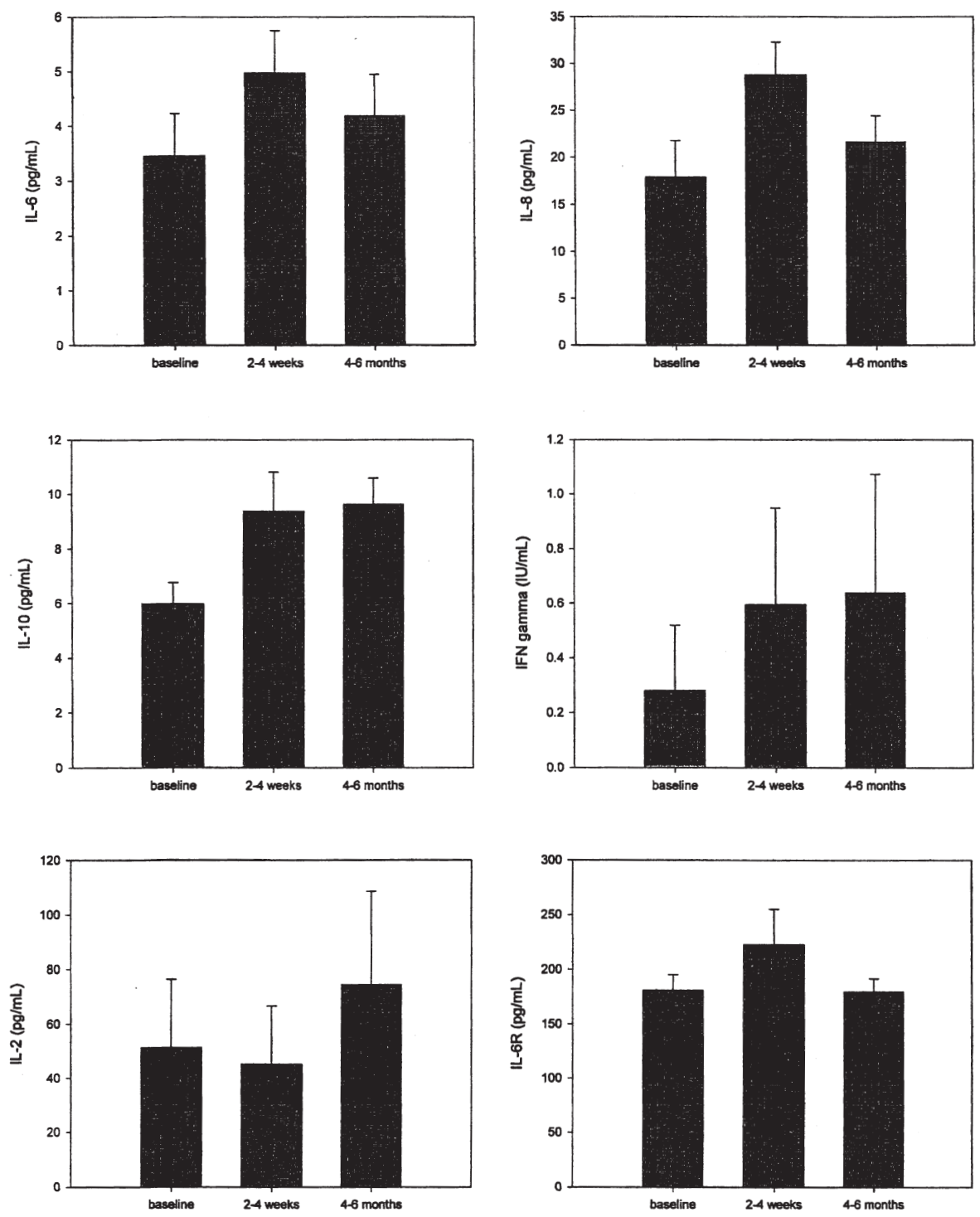

Fig. 2. Effects of interferon-alpha immunotherapy on serum interleukin-6 (IL-6), IL-8, IL-10, IFN-gamma, IL-2 and IL-6 receptor (IL-6R) in 14 patients with hepatitis-C, in baseline conditions (basal), and 2-4 weeks and 4-6 months after starting immunotherapy.

The results of RM design ANOVAs are described in Section 3. 
(1) IL-6 was significantly $(F=12.4$, d.f. $=1 / 36$, $P=0.001)$ greater in subjects with a higher MADRS score (mean $5.6 \pm 2.8 \mathrm{pg} / \mathrm{ml}$ ) than with a lower MADRS score $($ mean $=2.8 \pm 2.3 \mathrm{pg} / \mathrm{ml})$; and (2) IL-8 was significantly greater $(F=5.7$, d.f. $=1 / 36, P=0.02)$ in subjects with a higher MADRS score (mean $27.4 \pm 14.9 \pm 2.8 \mathrm{pg} / \mathrm{ml}$ ) than with a lower MADRS score (mean $=18.2 \pm$ $9.9 \mathrm{pg} / \mathrm{ml}$ ).

\section{Discussion}

The major findings of this study are that treatment with IFN $\alpha$ significantly induces the cytokine network and that the therapy-induced increases in some of the pro-inflammatory cytokines, in particular IL-6, are significantly and positively related to therapy-induced increases in MADRS and HAM-A scores.

The findings that immunotherapy with IFN $\alpha$ increases the serum concentrations of IL- 6 , IL-8 and IL-10 suggest that IFN $\alpha$-based immunotherapy induces a stimulation of the cytokine network, suggesting Th-1-like (increased serum IFN $\gamma$ ), Th-2-like (increased IL-10) and monocytic cell (increased serum IL-6) activation (Cavaillon, 1996). These results are in agreement with previous studies reporting that immunotherapy with IFN $\alpha$ induces the production of other cytokines (see Section 1). Nevertheless, the findings reported in the literature are sometimes contradictory. For example, most but not all studies reported increased serum IL- 6 concentrations during IFN $\alpha$-based immunotherapy (Shimizu et al., 1995; Grungreiff et al., 1999; Malaguarnera et al., 1997). The increases in serum IL-8 following IFN $\alpha$-based immunotherapy also indicate activation of the cytokine network. IL-8 is produced by monocytes, macrophages, neutrophils and endothelial cells after stimulation by IL- 1 and TNF $\alpha$ (Cavaillon, 1996). IL-8 induces the chemotaxic activity in neutrophils, lymphocytes, natural killer cells, eosinophils and basophils (Cavaillon, 1996). Previous findings also point toward activation of the immune system following IFN $\alpha$-based therapy. For example, Quiroga et al. (1994) reported increased serum sIL-2R, CD8 and neopterin con- centrations during IFN $\alpha$-based immunotherapy. We found significant and consistent increases in serum IL-10 concentrations during the 6-month treatment period with IFN $\alpha$. Not all authors were able to find such changes in IL-10 production. Thus, Fabris et al. (1999) reported that serum IL-10 concentrations fluctuated with no significant changes at any time point during the first 24 $h$ after one dose of IFN $\alpha 2 b$ ( 3 MUI s.c.). Cacciarelli et al. (1996) measured serial IL-10 levels in a subset of 11 patients at baseline and 12 weeks after IFN $\alpha$ therapy and found that treatment with IFN $\alpha$ decreased the levels of IL-10. Cramp et al. (2000) reported that treatment-induced control of hepatitis-C viremia is associated with the development of HCV-specific T-cell responses with enhanced IFN $\gamma$ and low IL-10 production. However, while previous studies aimed to examine the effects of IFN $\alpha$ immunotherapy on the cytokine network, the main aim of the present study was to examine the effects of IFN $\alpha$ on the cytokine network in relation to the development of depressive/anxiety symptoms.

Another major finding of this study is that prolonged treatment with IFN $\alpha$ has immunoregulatory effects, which are time-dependent. Thus, the stimulating effects of IFN $\alpha$-based immunotherapy on serum IL- 6 and IL- 8 are found 2-4 weeks, but not 4-6 months, after starting IFN $\alpha$ treatment. Prolonged treatment with IFN $\alpha$, on the other hand, increases serum IL-10 during the whole study period. Nevertheless, there was a trend toward increased serum IL-6, IL-8 and IFN $\gamma$ concentrations 4-6 months after starting immunotherapy. Thus, from our results it appears that IFN $\alpha$-based immunotherapy induces the cytokine network as a whole rather than Th- 1 vs. Th-2 cytokine production. Previous research, however, has shown that treatment with IFN $\alpha$ may diminish the Th-2-like cytokine response (Cacciarelli et al., 1996). The latter authors found that treatment with IFN $\alpha$ for 12 weeks decreased the levels of the Th-2-like cytokines, i.e. IL-4 and IL-10, which paralleled the decrease in HCV RNA. Since Th-1-like cytokines are required for host antiviral immune responses, including cytotoxic T-cell generation and natural killer cell activation, and Th-2-like cytokines (IL-4, IL-10) can 
inhibit the development of these effector mechanisms, these authors concluded that modulation of T-cell function and cytokine production may be one mechanism whereby IFN $\alpha$ therapy results in reduced viral burden (Cacciarelli et al., 1996). In another study, Cramp et al. (2000) concluded that the greater efficacy of the combination therapy of IFN $\alpha$ plus ribavirin may be related to its ability to suppress $\mathrm{HCV}$-specific IL-10 production. Harraga et al. (1999) reported a switch from a Th-2like to a Th-1-like cytokine profile after IFNo monotherapy for human echinococcosis. One possible explanation for the above contradictory results is that the capacity of IL-10 production varies according to the genetic composition of the IL-10 locus (Edwards-Smith et al., 1999), while in most studies (including ours) the IL-10 gene promotor polymorphism was not determined.

A third major finding of the present study is the significant and positive relationship between the IFN $\alpha$-induced activation of the cytokine network and the depression and anxiety scores. As described in Section 1, IFN $\alpha$-based immunotherapy is known to induce depressive and anxiety symptoms and even full-blown major depression in a considerable number of patients (Bonaccorso et al., 2000, 2001). In the present study we found significant correlations between the IFN $\alpha$-induced changes in serum IL- 6 and IL-8, but not IL-10, and those in the MADRS or the HAM-A scores. The results that the changes in serum IL-6 from baseline to 2-4 weeks later are significantly and positively correlated to the changes in the MADRS from baseline to 4-6 months later suggest that the IFN $\alpha$-induced increase in serum IL-6 may predict the severity of depressive symptoms. These findings are in accordance with the known behavioral effects of IL-6. Thus, sustained administration of IL-6, produced by infecting healthy MRL $+/+$, C 3 H.SW and Balb $/ C$ mice with adenovirus vector carrying cDNA for murine IL-6, resulted in increased serum IL-6 levels over 5 days, and a rapid decline in preference for sucrose. These findings suggest that sustained IL-6 production is an early mechanism in the behavioral and motivation/emotional alterations during chronic inflammatory conditions (Sakic et al.,
1997). In humans, a low dose of IL-6 $(0.5 \mu \mathrm{g} / \mathrm{kg}$ body wt.) is associated with increased ratings of fatigue, inactivity and difficulties concentrating (Spath-Schwalbe et al., 1998). Thus, the depressogenic effects of IFN $\alpha$ could in part be related to its stimulatory effect on the production of proinflammatory cytokines (see Section 1). Moreover, IFN $\alpha$ 's depressogenic properties can also be related to changes in the serotonergic system (Bonaccorso et al., 2001), which are known to play a role in the pathogenesis of depression (Maes and Meltzer, 1995). (1) IFN $\alpha$ administration may increase the 5-HT transporter mRNA and the uptake activity of the 5-HT transporter (Morikawa et al., 1998). (2) IFN $\alpha$ administration has a suppressant effect on the serotonin concentrations in brain and serum (Kamata et al., 2000). (3) IFN $\alpha$ immunotherapy significantly induces the catabolism of tryptophan (Bonaccorso et al., 2001).

There are, however, a number of limitations to the interpretation of the results. (1) Since the design of the study is cross-sectional and involves the assessment of current symptomatology coupled with immunological changes, there needs to be caution about causality. (2) For future evaluations it would be helpful to introduce an additional time point after termination of the IFN $\alpha$ therapy. (3) Due to the low number of patients included here we were unable to perform an item-by-item analysis of the IFN $\alpha$-induced changes in the MADRS and HAM-A depressionanxiety symptoms in relation to the stimulation of the cytokine network. Nevertheless, we have shown that in 30 patients affected by chronic active $C$-hepatitis immunotherapy with IFN $\alpha$ for 3 months significantly increased the MADRS items sadness (expressed and unexpressed); irritability; insomnia; loss of appetite; and asthenia (Bonaccorso et al., 2001).

\section{Acknowledgements}

This work was supported by a grant from the National Council for Research n.203.04.17 of 09/06/97. 


\section{References}

Anisman, H., Kokkinidis, L., Borowski, T., Merali, Z., 1998 Differential effects of interleukin (IL)-1beta, IL-2 and IL-6 on responding for rewarding lateral hypothalamic stimulation. Brain Research 779, 177-187.

Bluthé, R.M., Crestani, F., Kelley, K.W., Dantzer, R., 1992 Mechanisms of the behavioral effects of interleukin 1. Annals of the New York Academy of Sciences 650, 268-275.

Bonaccorso, S., Meltzer, H.Y., Maes, M., 2000. Psychological and behavioral effects of interferons. Current Opinion in Psychiatry 13, 673-677.

Bonaccorso, S., Marino, V., Biondi, M., Grimaldi, F., Ippoliti, F., Maes, M., 2001. Major depression induced by interferon-alpha in patients affected by hepatitis $\mathrm{C}$ virus. Journal of Affective Disorders (in press).

Cacciarelli, T.V., Martinez, O.M., Gish, R.G., Villanueva, J.C Krams, S.M., 1996. Immunoregulatory cytokines in chronic hepatitis $C$ virus infection: pre- and posttreatment with interferon alpha. Hepatology 24, 6-9.

Cavaillon, J.-M., 1996. Les Cytokines. Masson, Paris.

Connor, T.J., Song, C., Leonard, B.E., Merali, Z., Anisman, H., 1998. An assessment of the effects of central interleukin-1beta, $-2,-6$, and tumor necrosis factor-alpha administration on some behavioural, neurochemical, endocrine and immune parameters in the rat. Neuroscience 84 , 923-933.

Cramp, M.E., Rossol, S., Chokshi, S., Carucci, P., Williams, R. Naoumov, N.V., 2000. Hepatitis C virus-specific T-cell reactivity during interferon and ribavirin treatment in chronic hepatitis C. Gastroenterology 118, 346-355.

Edwards-Smith, C.J., Jonsson, J.R., Purdie, D.M., Bansal, A., Shorthouse, C., Powell, E.E., 1999. Interleukin-10 promote polymorphism predicts initial response of chronic hepatitis C to interferon alpha. Hepatology 30, 526-530.

Fabris, C., Del Forno, M., Falleti, E., Toniutto, P., Pirisi, M., 1999. Kinetics of serum soluble tumour necrosis factor receptor (TNF-R) type-I and type-II after a single interferon-alpha (IFN-alpha) injection in chronic hepatitis C. Clinical and Experimental Immunology 117, 556-560.

Grungreiff, K., Reinhold, D., Ansorge, S., 1999. Serum concentrations of sIL-2r, IL-6, TGF-beta1, neopterin, and zinc in chronic hepatitis $C$ patients treated with interferon-alpha. Cytokines 11, 1076-1080.

Hamilton, M., 1959. The assessment of anxiety by rating. British Journal of Psychiatry 32, 50-55.

Harraga, S., Godot, V., Bresson-Hadni, S., Pater, C., Beurton, I., Bartholomot, B., Vuitton, D.A., 1999. Clinical efficacy of and switch from $T$ helper 2 to $T$ helper 1 cytokine profile after interferon alpha2a monotherapy for human echinococcosis. Clinical Infectious Diseases 29, 205-206.

Kamata, M., Higuchi, H., Yoshimoto, M., Yoshida, K., Shimizu, T., 2000. Effect of single intracerebroventricular injection of alpha-interferon on monoamine concentration in the rat brain. European Neuropsychopharmacology 10, 129-132.

Kawamura, C., Nakajima, S., Kuroki, T., Monna, T., 1999. Two-dimensional analysis of production of IL-6 and TNF- alpha can predict the efficacy of IFN-alpha therapy. Hepatogastroenterology 46, 2941-2945.

Linthorst, A.C. Reul, J.M, 1998. Brain neurotransmission during peripheral inflammation. Annals of the New York Academy of Sciences 840, 139-152.

Maddrey, W.C., 1999. Safety of combination interferon alfa$2 \mathrm{~b} /$ ribavirin therapy in chronic hepatitis C-relapsed treatment-naive patients. Seminars in Liver Diseases 19, 67-75.

Maes, M., Meltzer, H.Y.M., 1995. The serotonin hypothesis of major depression. In: Bloom, F., Kupfer, D. (Eds.), Psychopharmacology: The Fourth Generation of Progress. Raven Press, New York, pp. 933-944.

Maes, M., Capuron, L., Ravaud, A., Gualde, N., Bosmans, E., Egyed, B., Dantzer, R., Neveu, P.J., 2001. Lowered serum dipeptidyl peptidase IV activity is associated with depressive symptoms and cytokine production in cancer patients receiving interleukin-2-based immunotherapy. Neuropsychopharmacology 24, 130-140.

Maier, S.F., Watkins, L.R., 1998. Cytokines for psychologists: implications of bidirectional immune-to-brain communication for understanding behavior, mood, and cognition. Psychological Reviews 105, 83-107.

Malaguarnera, M., Di Fazio, I., Laurino, A., Ferlito, L., Romano, M., Trovato, B.A., 1997. Serum interleukin 6 concentrations in chronic hepatitis $\mathrm{C}$ patients before and after interferon-alpha treatment. International Journal of Clinical Pharmacology and Therapy 35, 385-388.

Montgomery, S.A., Åsberg, A., 1979. A new depression scale designed to be sensitive to change. British Journal of Psychiatry 134, 382-389.

Morikawa, O., Sakai, N., Obara, H., Saito, N., 1998. Effects of interferon-alpha, interferon-gamma and cAMP on the transcriptional regulation of the serotonin transporter. European Journal of Pharmacology 349, 317-324.

Naveau, S., Balian, A., Degos, F., Daurat, V., Chevret, S. Gayno, S., Bastie, A., Riachi, G., Bartolomei-Portal, I, Barange, K., Moussalli, J., Bailly, F., Chaumet-Riffaud, P., Emilie, D., 1999. Prognostic value of the soluble interleukin-2 receptor in chronic hepatitis $\mathrm{C}$ treated with interferon-alpha. Multicenter GER-CYT 04 Group. Journal of Hepatology 31, 612-617.

Quiroga, J.A., Martin, J., Pardo, M., Carreno, V., 1994. Serum levels of soluble immune factors and pathogenesis of chronic hepatitis $\mathrm{C}$, and their relation to therapeutic response to interferon-alpha. Digest of Diseases and Science $39,2485-2496$.

Sakic, B., Szechtman, H., Braciak, T., Richards, C., Gauldie, J., Denburg, J.A., 1997. Reduced preference for sucrose in autoimmune mice: a possible role of interleukin-6. Brain Research Bulletin 44, 155-165.

Shimizu, H., Ohtani, K., Sato, N., Nagamine, T., Mori, M., 1995. Increase in serum interleukin-6, plasma ACTH and serum cortisol levels after systemic interferon-alpha administration. Endocrinology Journal 42, 551-556.

Spath-Schwalbe, E., Hansen, K., Schmidt, F., Schrezenmeier, H., Marshall, L., Burger, K., Fehm, H.L., Born, J., 1998 Acute effects of recombinant human interleukin-6 on en- 
S. Bonaccorso et al. / Psychiatry Research 105 (2001) 45-55

docrine and central nervous sleep functions in healthy men. Journal of Clinical Endocrinology and Metabolism 83, 1573-1579.

Taylor, J.L., Grossberg, S.E., 1998. The effects of interferonalpha on the production and action of other cytokines. Seminars in Oncology 25 (1 Suppl 1), 23-29.
Tsai, S.L., Liaw, Y.F., Chen, M.H., Huang, C.Y., Kuo, G.C., 1997. Detection of type 2 like T-helper cells in hepatitis C virus infection: implication for hepatitis $C$ virus chronicity. Hepatology 25, 449-458.

Yirmiya, R., 1996. Endotoxin produces a depressive-like episode in rats. Brain Research 711, 163-174. 


\section{Chapter 6}

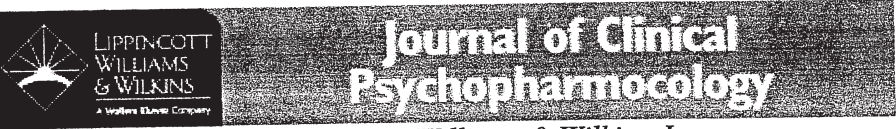

(C) 2002 Lippincott Williams \& Wilkins, Inc.

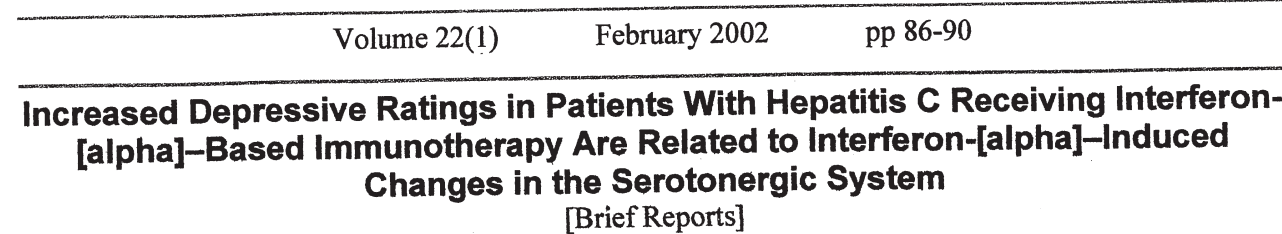

Bonaccorso, Stefania MD*†‡; Marino, Valentina MD*; Puzella, Antonella MD*; Pasquini, Massimo MD*; Biondi, Massimo MD, PhD, Prof*; Artini, Marco MD§; Almerighi, Cristiana MD§; Verkerk, Robert RT[/]; Meltzer, Herbert MD, Prof;; Maes, Michael MD, PhD, Prof $\$$

*Psychiatric Hospital, University La Sapienza, Rome, Italy; †Department of Psychiatry, University Hospital of Maastricht, Paastricht the Netherlands; tDepartment of Psychiatry, Vanderbilt University, Nashville, Tennessee; §Department of Hepatology, I Medical Clinic, University La Sapienza, Rome, Italy; [//]Department of Medical Biochemistry, University of Antwerp, Belgium Received June 30, 2000; accepted after revision March 26, 2001.

Received June 30, 2000; accepted after revision March 26, 2001.
Address requests for reprints to: Michael Maes, MD, PhD, Department of Psychiatry and Neuropsychology, University Hospital of Address requests for reprints to: Michael Maes, MD, PhD, Department of Psychiatry and Postbus 5800, 6202 AZ Maastricht, The Netherlands. Address e-mail to: crc-mh@online.be.

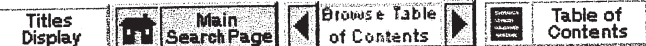

Outline

- Abstract

- Methods

- Patients

- Methods

- Statistics

- Results

- Discussion

- Acknowledgment

- References

\section{Graphics}

- Table 1

\begin{tabular}{|l|}
\hline Output... \\
\hline Print Preview \\
Email Article Text \\
Save Article Text \\
Full Text (PDF) \\
\hline Links... \\
\hline Help \\
Logoff \\
\hline History... \\
\hline hcreased Depressive Rati... \\
\hline
\end{tabular}

\footnotetext{
Abstract \pm

There is now evidence that repeated administration of interferon-[alpha] (IFN-[alpha]) to patients with chronic active hepatitis and cancers induces depressive symptoms. There is also evidence that induction of the cytokine network modulates the serotonergic system and that major depression is related to activation of the cytokine network and disturbances in the serotonergic metabolism. The aims of this study were to examine the effects of IFN-[alpha]-based immunotherapy on the development of depressive symptoms in relation to its effects on plasma tryptophan and kynurenine and serum serotonin (5-HT). Eighteen patients affected by chronic active hepatitis $C$ were treated with IFN-[alpha] (3-6 million units subcutaneously three to six times a week for 6 months) and had measurements of the previous parameters before starting immunotherapy and 2, 4, 16, and 24 weeks later. Severity of depression and anxiety were measured with the Montgomery-Asberg Depression Rating
} 
Scale (MADRS) and the Hamilton Rating Scale for Anxiety (HAM-A) scale, respectively. Immunochemotherapy with IFN-[alpha] (1) significantly increased the MADRS and HAM-A scores and serum kynurenine concentrations and (2) significantly reduced plasma tryptophan and serum 5-HT concentrations. IFN-[alpha]based immunotherapy significantly increased the kynurenine per tryptophan quotient, which estimates the activity of indoleamine 2,3-dioxygenase, the major tryptophan-catabolizing enzyme, which is induced by IFNs. There are significant relationships between the IFN-[alpha]-induced changes in the MADRS score and serum kynurenine (positive) and 5-HT (negative) concentrations. Immunotherapy with IFN-[alpha] significantly increases the severity of depressive symptoms. The latter is related to changes in the serotonergic system, such as depletion of serum 5-HT and induction of the catabolism of tryptophan to kynurenine. It is suggested that the IFN-[alpha]induced changes in the serotonergic turnover could play a role in the development of IFN-[alpha]-induced depressive symptoms.

IMMUNOTHERAPY WITH interferon-[alpha] (IFN-[alpha]) is used for treating patients with chronic hepatitis C. 1 IFN-[alpha] is a cytokine with potent antiviral and antiproliferative effects. $\underline{2}$ The adverse effects of IFN[alpha]-based immunotherapy, which appear after several weeks, comprise major depression and depressive symptoms, such as expressed and unexpressed sadness, irritability, insomnia, loss of appetite, asthenia, loss of interest, slowness, anxiety, social withdrawal, and lack of concentration. 1

There is now evidence that major depression is associated with an activation of the inflammatory response system (IRS), as indicated by (1) increased numbers of peripheral blood neutrophils, monocytes, and activated T cells; (2) increased plasma concentrations of positive acute phase proteins (APPs), such as haptoglobin, and decreased plasma concentrations of negative APPs, such as albumin and transferrin; and (3) increased production of proinflammatory cytokines, such as interleukin-1[beta] (IL-1[beta]), IL-6, and IFN-[gamma]. 3,4 IRS activation and administration of proinflammatory cytokines may induce depressive symptoms through their modulation of, among other things, the central and peripheral serotonergic system. 3.5 Major depression is accompanied by disorders in the peripheral and central metabolism of serotonin (5-HT), e.g., decreased activity of the central presynaptic 5-HT neuron, which is, in part, related to a decreased availability of plasma tryptophan (the precursor of 5-HT), and changes in postsynaptic receptors such as 5-HT2A and 5-HT1A receptors. $\underline{6}$ It has been hypothesized that, in depression, reduced plasma tryptophan is related to activation of the IRS. 7,8 Indeed, highly significant inverse relationships were found between plasma tryptophan and indicators of IRS activation, such as increased plasma haptoglobin and production of IL-6. 7,8 One explanation is that indoleamine 2,3dioxygenase (IDO), a major tryptophan-catabolizing enzyme, is induced during IRS activation by proinflammatory cytokines, such as IFN-[gamma] and IL-2. 9-11 Treatment of rats with IFN-[alpha] significantly reduces in a dosedependent manner 5-HT in the frontal cortex, midbrain, and striatum. 12 Treatment with IFN[alpha] and IFN-[gamma] of human placental choriocarcinoma cells increases the 5-HT transporter messenger RNA (mRNA) and the uptake activity of the 5-HT transporter. 13 These results suggest that changes in the turnover of 5-HT are a possible neurochemical mechanism of depression induced by IFN-[alpha]-based immunotherapy. However, no research has examined the effects of IFN-[alpha]-based immunotherapy on plasma tryptophan in relation to its major catabolite in the IDO pathway, i.e., kynurenine, and on serum 5-HT concentrations.

The aims of this study were to examine (1) whether immunotherapy with IFN-[alpha] in patients with hepatitis C decreases serum 5-HT concentrations and induces the catabolism of tryptophan and, subsequently, causes reductions in plasma tryptophan and increases in serum kynurenine; and 2) whether there are associations between the immunotherapy-induced changes in depressive symptoms and in the metabolism of 5-HT.

\section{Methods \pm}

Patients 2

Eighteen patients ( 10 men and 8 women; mean age $\pm S D, 40.5 \pm 11.5$ years) affected by chronic active hepatitis $C$ were recruited from the hepatology clinic at the I Medical Clinic, University of Rome La Sapienza from November 1997 to May 1998. Three of the 18 patients suspended IFN-[alpha] therapy soon after starting treatment, one because of a reduction in the number of platelets and two because of low IFN-[alpha] compliance. One patient did not have baseline measurements. Consequently, 14 patients ( 8 men and 6 women; mean age \pm $\mathrm{SD}, 41.7 \pm 10.8$ years) completed the IFN-[alpha] treatment. The diagnosis of chronic active hepatitis $\mathrm{C}$ was based on persistently increased transaminase values (1.5 over the normal range for at least 6 months), antihepatitis $\mathrm{C}$ virus $(\mathrm{HCV})$ positivity, and histologic report. Inclusion criteria were age less than 65 years; histologic 
diagnosis of chronic hepatitis; positivity for anti-HCV antibodies and HCV RNA in serum; and platelet counts greater than $100,000 / \mathrm{mm}^{3}$. Exclusionary criteria for patients were liver cirrhosis; heart, kidney, or autoimmune diseases; infection with hepatitis B or D virus or HIV; white blood cell counts less than $4,000 / \mathrm{mm}^{3}$; prothrombin time less than $60 \%$; chronic alcohol abuse or active intravenous drug use; and a past or present history of axis I disorders, such as dysthymia or major or bipolar depression. Illness onset and awareness ranged from a minimum of 1 to a maximum of 2 years. Before starting IFN-[alpha]-based immunotherapy, none of the patients was taking psychotropic drugs, such as antidepressants, neuroleptics, or benzodiazepines. Moreover, patients did not take any other medication besides IFN-[alpha] during the study period, and no changes in the physical status were recorded during the treatment period. The local ethical committee approved the study design, and the subjects gave written informed consent after the study protocol was fully explained.

Methods \pm

Patients eligible for IFN-[alpha] treatment were randomized in a pharmacologic trial to receive one of two treatment schedules with IFN-[alpha]-2A (Roferon; Roche, Summerville, NJ): schedule A (N=10), 6 million units IFN-[alpha] subcutaneously per day, 6 days a week for 4 weeks; and schedule $B(N=4), 6$ million units IFN subcutaneously thrice in a week for 4 weeks. Because there were no significant differences in any of the clinical ratings and biologic variables between the treatment groups at any of the time points, we performed further statistical analyses on both treatment groups combined. Both groups continued therapy with 6 million units IFN subcutaneously thrice in a week for 12 weeks. Responders at 4 months received 3 million units IFN subcutaneously thrice in a week for a further 8 months. Fasting (overnight) plasma or serum for the assay of amino acids, 5-HT, and kynurenine was always collected at 8:00 a.m. at baseline (before starting treatment) and $2,4,16$, and 24 weeks after starting IFN-[alpha]-based immunotherapy. During each of these sessions, patients completed the Montgomery-Asberg Depression Rating Scale (MADRS) 14 and the Hamilton Rating Scale for Anxiety (HAM-A). 15

High-performance liquid chromatography (HPLC) was used to measure plasma tryptophan as well as the amino acids known to compete with the same cerebral transport system, i.e., valine, leucine, tyrosine, phenylalanine, and isoleucine. 8,16 The tryptophan/valine + leucine + isoleucine + tyrosine + phenylalanine (CAA) was computed and multiplied by 100 as an index of the availability of plasma tryptophan to the brain. 8 Serum 5-HT (Biorad, Eke, Nazareth, Belgium) was determined by means of HPLC. 17 Serum kynurenine was determined by means of HPLC as described by Holmes. 18 The kynurenine per tryptophan quotient $(\mathrm{K} / \mathrm{T})$ was computed, because this ratio allows us to estimate IDO activity. 19 To minimize the analytical variability, all assays were performed in a single run with a single lot number of reagents and consumables used by a single operator. The intra-assay coefficient of variation values were tryptophan $3.3 \%$; tyrosine $3.8 \%$; valine $3.0 \%$; phenylalanine $3.2 \%$; isoleucine $3.4 \%$; leucine $3.7 \%$; 5-HT $6.0 \%$; and kynurenine $4.5 \%$.

Statistics $v$

Group mean differences were ascertained by means of analysis of variance (ANOVA). Repeated measure (RM) design ANOVAs were used to check the effects of IFN-[alpha] immunotherapy on the clinical and biochemical measurements. We used the RM ANOVA mixed model to check the overall effects of IFN-[alpha] on the measurements (results not shown). There were no significant differences in any of the variables, either between weeks 2 and 4 or between months 4 and 6 . To check the early (first month) versus protracted (several months) effects of IFN-[alpha], we introduced the peak/nadir values obtained at weeks 2 and 4 and the peak/nadir values obtained at moths 4 and 6 for those variables that significantly increased and decreased, respectively. Thus, RM design ANOVAs were performed on the baseline values and the measurements after 2 to 4 weeks and 4 to 6 months and corrected for sphericity. A priori comparisons among treatment means were ascertained with the Dunn test. $\underline{2}$ Tests on simple effects were performed to examine significant main effects or significant interaction patterns. 20 Correlations between variables were assessed by means of Pearson's product moment correlation coefficients. Time relationships were computed by means of regression analyses, which were pooled over the repeated measurements in the subjects. This method eliminates the interindividual variability and assesses the relationships over time between variables.

Results 4$]$

Table 1 shows that the MADRS and HAM-A scores were significantly different among the three time points. We have divided the patients into two subgroups: those whose MADRS scores increased to or more than $15(\mathrm{~N}=7$; MADRS responders) and those whose MADRS scores remained low ( $N=7$; MADRS nonresponders) during IFN-[alpha] immunotherapy (Fig. 1). RM design ANOVA with the MADRS responder status as factor shows that there is a significant difference in the MADRS score among the three time points $(F[2,22]=6.2, p=0.007)$ and a 
trend toward a significant time $\times$ MADRS status interaction $(F[2,22]=2.6, p=0.09)$. Analyses on simple effects show (1) significant effects of time in MADRS responders $(F[2,24]=7.9, p=0.002)$, but not in nonresponders $(F[2,24]=0.9, p=0.6)$, and (2) significantly higher MADRS scores in MADRS responders than in nonresponders 2 to 4 weeks $(F[1,36]=13.5, p=0.001)$ and 4 to 6 months $(F[1,36]=7.4, p=0.009)$ after starting immunotherapy, but not at baseline $(F[1,36]=0.3, p=0.6)$. The Dunn test showed (tested at $p=0.0166$ after $p$ correction) significantly higher MADRS scores 2 to 4 weeks $(t=2.64, p=0.013)$ and 4 to 6 months $(t=$ 3.33, $p=0.003$ ) after starting immunotherapy than before and no differences between weeks 2 and 4 versus months 4 to $6(t=0.7, p=0.5)$. A RM design ANOVA performed on the HAM-A scores (Fig. 1) and with the MADRS responder status as factor showed that there was a significant effect of time $(F[2,21]=4.2, p=0.02)$ but no significant time $\times$ MADRS status interaction $(F[2,21]=1.1, p=0.3)$. Dunn tests showed a trend toward a significantly higher HAM-A score 2 to 4 weeks after starting IFN-[alpha] therapy $(t=2.31, p=0.027)$ and significantly higher HAM-A scores 4 to 6 months $(t=2.69, p=0.012)$ after starting immunotherapy than before.

\begin{tabular}{lcccccc}
\hline Variables & Baseline & $2-4$ weeks & $4-6$ months & $F^{i}$ & $d f$ & $p$ \\
\hline VIADRS & $4.7(4.0)$ & $10.4(9.4)$ & $11.1(7.7)$ & 4.3 & 2.25 & 0.02 \\
IAM-A & $6.0(5.0)$ & $9.9(1.8)$ & $10.1(6.7)$ & 3.7 & 2.24 & 0.03 \\
5-HT & $595(251)$ & $361(130)$ & $528(128)$ & 39.8 & 1.17 & $<10^{-1}$ \\
Tryptophan $(\mu \mathrm{M})$ & $60.2(6.2)$ & $55.4(12.3)$ & $50.8(10.8)$ & 8.9 & 2.24 & 0.002 \\
Kynurenine $(\mu \mathrm{M})$ & $2.29(0.74)$ & $3.26(1.09)$ & $2.90(0.88)$ & 38.4 & 2.2 & $<10^{-5}$ \\
KT quotient & $0.039(0.014)$ & $0.063(0.033)$ & $0.061(0.031)$ & 49.1 & 2.23 & $<10^{-1}$ \\
CAA $(\mu \mathrm{M})$ & $594(61)$ & $561(124)$ & $5232(134)$ & 4.8 & 1.19 & 0.03 \\
tryptophan/CAA X 100 & $10.17(0.87)$ & $9.92(1.30)$ & $9.66(1.04)$ & 0.8 & 2.25 & 0.6 \\
\hline
\end{tabular}

*All results are shown as mean (SD).

All results of repeated measure design ANOVA (the results were adjusted for sphericity).

Table 1. Measurements of the Montgomery Asberg Depression Rating Scale (MADRS) and the Hamilton-Anxiety (HAM-A) scale, serum serotonin (5-HT), plasma tryptophan, serum kynurenine and the kynurenine per tryptophan (K/T) quotient, the sum of the 5 competing amino acids (CAA) and the tryptophan/CAA ratio in hepatitis $C$ patients before starting treatment (baseline), and after 2-4 weeks and 4-6 months of interferon-alpha-based immunotherapy*All results are shown as mean (SD). ${ }^{*}$ All results of repeated measure design ANOVA (the results were adjusted for sphericity). 

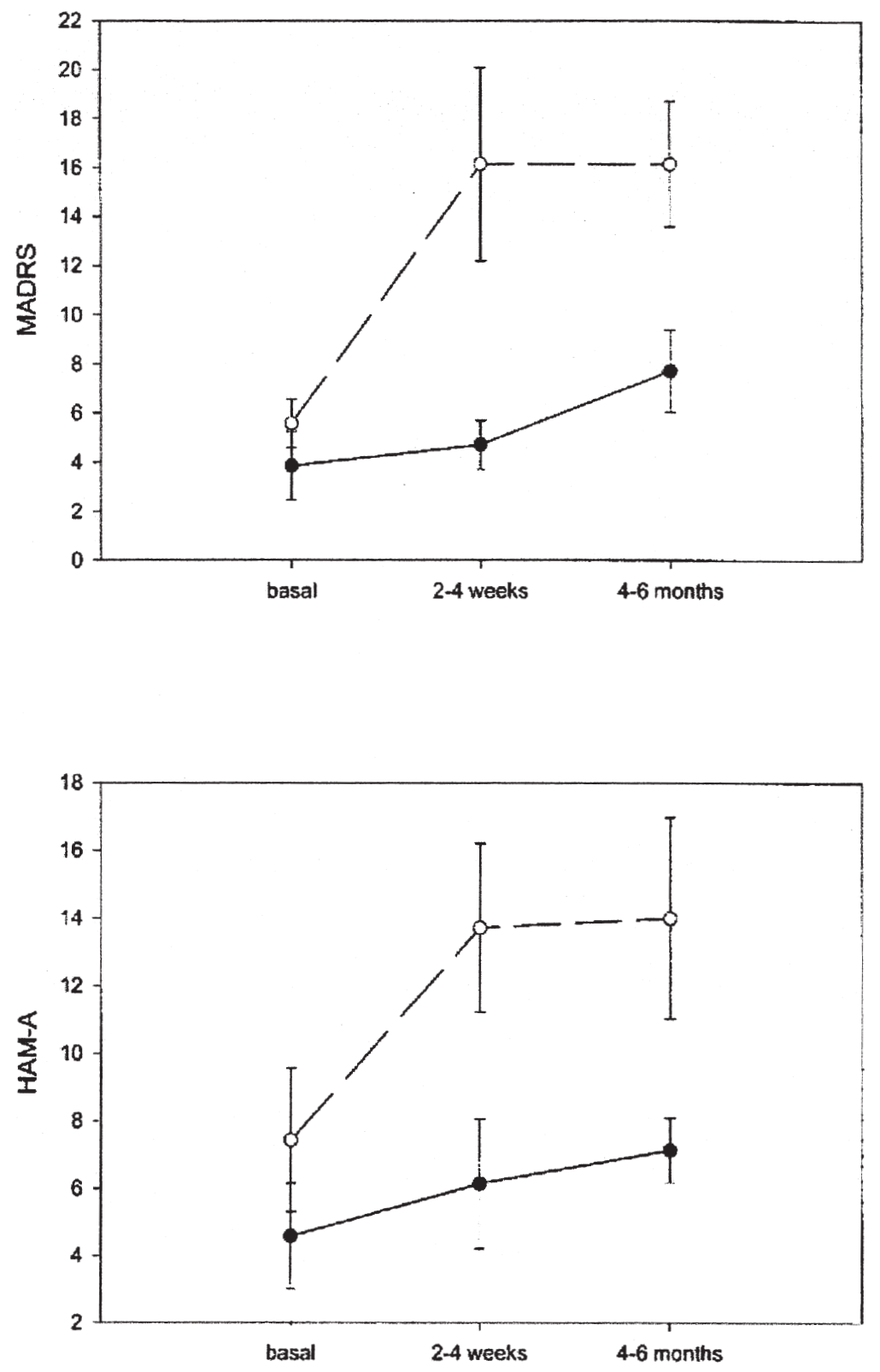

Fig. 1. Effects of IFN-[alpha] immunotherapy on the MADRS and the HAM-A scale in 14 patients with hepatitis $\mathrm{C}$. The patients were divided into those whose MADRS scores increased to or more than 15 ([heavy horizontal bar, white circle, heavy horizontal bar]) and those whose MADRS scores remained low ([heavy horizontal bar, black circle, heavy horizontal bar]) during IFN-[alpha] immunotherapy.

Table 1 shows significant effects of IFN-[alpha] on serum 5-HT, plasma tryptophan, serum kynurenine, the K/T quotient, and plasma CAA. Dunn tests showed (1) significantly lower serum 5-HT 2 to 4 weeks $(t=6.93, p=$ 
$0.000008)$ and 4 to 6 months $(t=8.34, p=0.000002)$ after starting treatment than before, but no significant differences between the two posttreatment time points $(t=1.42, p=0.2)$; (2) significantly lower plasma tryptophan 4 to 6 months $(t=3.97, p=0.0008)$, but not 2 to 4 weeks $(t=2.07, p=0.05)$, after starting treatment than before, and no significant differences between the two posttreatment time points $(t=1.90, p=0.07) ;(3)$ significantly higher serum kynurenine 2 to 4 weeks $(t=7.39, p=0.000005)$ and 4 to 6 months $(t=4.60, p=$ 0.0002 ) after starting treatment than before, and significantly higher values 2 to 4 weeks than 4 to 6 months after starting immunotherapy $(t=2.78, p=0.009) ;(4)$ a significantly higher $\mathrm{K} / \mathrm{T}$ quotient 2 to 4 weeks $(t=$ $5.08, p=0.0001)$ and 4 to 6 months $(t=4.74, p=0.0002)$ after starting treatment than before, and no significant differences between the two posttreatment time points $(t=0.33, p=0.7)$; and (5) significantly lower plasma CAA values 4 to 6 months $(t=3.11, p=0.005)$, but not 2 to 4 weeks $(t=1.74, p=0.09)$, after starting treatment than before, and no significant differences between the two posttreatment time points $(t=1.37, p=0.2)$. There were no significant differences in any of the variables between men and women, except for the CAA values, which were significantly $(F[1,36]=17.4, p=0.0003$ ) higher in men (mean, $615 \pm 113 \mu \mathrm{M} / \mathrm{L}$ ) than in women (mean, $493 \pm 61 \mathrm{M} / \mathrm{L}$ ). There were no significant correlations between patients' age and any of the pre- or posttreatment values of the clinical or biochemical data.

\section{Discussion:}

A first finding of this study is that immunotherapy with IFN-[alpha] significantly increased the MADRS and HAM-A scores within 1 month after starting therapy and that the depression and anxiety scores further increased during treatment. These findings are in agreement with previous reports showing that IFN-[alpha]based immunotherapy increases depressive ratings and induces full-blown major depression in a considerable number of patients. 1,21

The second major finding of this study is that serum 5-HT was significantly diminished during treatment with IFN-[alpha]. There is no evidence, however, that the plasma reductions observed here may translate to clinically significant reductions in brain 5-HT. Nevertheless, as explained in the introduction, IFN-[alpha] administration significantly reduces in a dose-dependent manner the 5-HT in the frontal cortex, midbrain, and striatum 12 and may increase 5-HT transporter mRNA and 5-HT transporter uptake activity. 13 Thus, these studies suggest that IFN-[alpha]-induced depressive symptoms may arise through depletion of peripheral and central 5-HT, which in part may be related to changes in the 5-HT transporter transcription and the uptake activity of the 5-HT transporter.

The third major finding of this study is that plasma tryptophan was significantly reduced and serum concentrations of kynurenine and the $\mathrm{K} / \mathrm{T}$ quotient were significantly increased during immunotherapy with IFN-[alpha], suggesting an increased catabolism of tryptophan to kynurenine. Indeed, IFN-[gamma], but also IFN-[alpha], may induce IDO. 22 Whereas IFN-[gamma] directly induces IDO, IFN-[alpha] has a weak direct effect and an indirect effect through a $15-\mathrm{kDa}$ protein, which is a product of IFN-[alpha]-treated monocytes and lymphocytes and which stimulates IDO and IFN-[gamma] production. 23 Because the production of IFN[gamma] is increased during immunotherapy with IFN-[alpha] (data not shown), the effects of IFN-[gamma] should also be considered. Indeed, activation of the IRS and disorders characterized by T helper cell type 1-like T-cell activation with hyperproduction of IFNs induce IDO and, consequently, stimulate the catabolism of tryptophan. 19 Decreased plasma tryptophan may play an important role in antimicrobial defenses and in the protection from attacks by autoreactive T cells through inhibition of T-cell proliferation. 24,25 A second mechanism may explain the decreased plasma tryptophan as well as CAA concentrations after IFN-[alpha]based immunotherapy. IRS activation, which follows IFN-[alpha] immunotherapy (Bonaccorso and colleagues, personal communication, 2001), involves a series of reactions in protein metabolism, such as increased use of amino acids, including tryptophan, by leukocytes and the liver, increased whole-body proteolysis, stimulation of the oxidation of amino acids, and higher uptake of the branched-chain amino acids (BCAA; valine, leucine, and isoleucine) by the liver and, thus, a redistribution of whole-body proteins. This state is characterized by decreased plasma amino acid concentrations and, in particular, of the BCAAs, and it is induced by proinflammatory cytokines. $\underline{7,8,26}$

Acknowledgment \pm

This work was supported by a grant from the National Council for Research (203.04.17) of September 6, 1997.

References \pm

1. Bonaccorso S, Meltzer HY, Maes M. Psychological and behavioral effects of interferons. Curr Opin Psychiatry 2000; 13:673-7. [Fulltext Link] [Context Link]

2. Cavaillon J-M, ed. Les cytokines. Paris: Masson, 1996. [Context Link] 
3. Maes M, Smith R, Scharpe S. The monocyte-T lymphocyte hypothesis of major depression. Psychoneuroendocrinology 1995; 20:111-6. [Medline Link] [PsycINFO Link] [BIOSIS Previews Link] [Context Link]

4. Maes M. Major depression and activation of the inflammatory response system. Adv Exp Med Biol 1999; 461:25-46. [Medline Link] [Context Link]

5. Yirmiya R. Behavioral and psychological effect of immune activation: implication for depression due to a general medical condition. Curr Opin Psychiatry 1997; 10:470-6. [Fulltext Link] [Context Link]

6. Maes M, Meltzer HYM. The serotonin hypothesis of major depression. In: Bloom FE, Kupfer DJ, eds. Psychopharmacology: the fourth generation of progress. New York: Raven Press, 1995:933-44. [Context Link]

7. Maes M, Meltzer HY, Scharpe S, et al. Relationships between lower plasma L-tryptophan levels and immune variables in depression. Psychiatr Res 1993; 49:11-27. [Medline Link] [PsycINFO Link] [Context Link]

8. Maes M, Wauters A, Verkerk R, et al. Lower L-tryptophan availability in depression: a marker of a more generalized disorder in protein metabolism. Neuropsychopharmacology 1996; 15:243-51. [Medline Link] [PsycINFO Link] [Context Link]

9. Brown RR, Lee CM, Kohler PC, et al. Altered tryptophan and neopterin metabolism in cancer patients treated with recombinant interleukin-2. Cancer Res 1989; 49:4941-4. [Medline Link] [BIOSIS Previews Link] [Context Link]

10. Takikawa O, Yoshida R, Yasui H, et al. The relationship between plasma kynurenine and indoleamine 2,3dioxygenase activity in the extrahepatic tissues. In: Schlossberger HG, Kochen W, Linzen B, Steinhart H, eds. Progress in tryptophan and serotonin research. Berlin: Walter de Gruyter and Co, 1984:517-20. [Context Link]

11. Moroni F, Russi P, Gallo-Mezo MA, et al. Modulation of quinolinic and kynurenic acid content in the rat brain: effects of endotoxins and nicotinylalanine. J Neurochem 1991; 57:1630-5. [Medline Link] [BIOSIS Previews Link] [Context Link]

12. Kamata $\mathrm{M}$, Higuchi $\mathrm{H}$, Yoshimoto $\mathrm{M}$, et al. Effect of single intracerebroventricular injection of alphainterferon on monoamine concentration in the rat brain. Eur Neuropsychopharmacol 2000; 10:129-32. [Medline Link] [PsycINFO Link] [Context Link]

13. Morikawa O, Sakai N, Obara H, et al. Effects of interferon-alpha, interferon-gamma and cAMP on the transcriptional regulation of the serotonin transporter. Eur J Pharmacol 1998; 349:317-24. [Medline Link] [BIOSIS Previews Link] [Context Link]

14. Montgomery SA, Asberg A. A new depression scale designed to be sensitive to change. Br J Psychiatry 1979; 134:382-9. [Medline Link] [PsycINFO Link] [BIOSIS Previews Link] [Context Link]

15. Hamilton M. The assessment of anxiety by rating. Br J Psychiatry $1959 ; 32: 50-5$. [Context Link]

16. Turnell D, Cooper J. Rapid assay for amino acids in serum or urine by precolumn derivatisation and reversedphase liquid chromatography. Clin Chem 1982; 28:527-31. [Medline Link] [BIOSIS Previews Link] [Context Link]

17. Croonenberghs J, Delmeire L, Verkerk R, et al. Peripheral markers of serotonergic and noradrenergic function in postpubertal, Caucasian males with autistic disorder. Neuropsychopharmacology 2000; 22:275-83. [Medline Link] [PsycINFO Link] [BIOSIS Previews Link] [Context Link]

18. Holmes EW. Determination of serum kynurenine and hepatic tryptophan dioxygenase activity by highperformance liquid chromatography. Anal Biochem 1988; 172:518-25. [Medline Link] [BIOSIS Previews Link] [Context Link]

19. Widner B, Sepp N, Kowald E, et al. Enhanced tryptophan degradation in systemic lupus erythematosus. Immunobiology 2000; 201:621-30. [Medline Link] [BIOSIS Previews Link] [Context Link]

20. Howell DC, ed. Statistical methods for psychology. Boston: Duxbury Press, 1982. [Context Link]

21. Bonaccorso S, Marino V, Biondi M, et al. Major depression induced by interferon-alpha in patients affected by hepatitis $\mathrm{C}$ virus. J Affect Disord 2001 (in press). [Context Link]

22. Taylor MW, Feng G. Relationship between interferon-gamma, indolamine 2,3-dioxygenase, and tryptophan catabolism. FASEB J 1991; 5:2516-22. [Medline Link] [BIOSIS Previews Link] [Context Link]

23. Recht M, Borden EC, Knight E. A human 15-kDa IFN-induced protein induces the secretion of IFN-gamma. J Immunol 1991; 147:2617-23. [Medline Link] [BIOSIS Previews Link] [Context Link]

24. Munn DH, Shafizadeh E, Attwood JT, et al. Inhibition of T cell proliferation by macrophage tryptophan catabolism. J Exp Med 1999; 189:1363-72. [Medline Link] [BIOSIS Previews Link] [Context Link] 
25. Mellor AL, Munn DH. Tryptophan catabolism and T-cell tolerance: immunosuppression by starvation? Immunol Today 1999; 20:469-73. [Medline Link] [BIOSIS Previews Link] [Context Link]

26. Darling G, Goldstein DS, Stull R, et al. Tumor necrosis factor: immune endocrine interaction. Surgery 1989; 106:1155-60. [Medline Link] [BIOSIS Previews Link] [Context Link] 


\section{Chapter 7}

\section{Treatment with interferon-alpha (IFN $\alpha$ ) of hepatitis C patients induces lower serum dipeptidyl peptidase IV activity, which is related to IFN $\alpha$-induced depressive and anxiety symptoms and immune activation}

M Maes ${ }^{1,2}, \mathrm{~S}$ Bonaccorso ${ }^{1,2,3}, V$ Marino $^{3}, \mathrm{~A} \mathrm{Puzella}^{3}, \mathrm{M}$ Pasquini ${ }^{3}, \mathrm{M}$ Biondi $^{3}, \mathrm{M}$ Artini $^{4}$, $\mathrm{C}$ Almerighi ${ }^{4}$ and $\mathrm{H}$ Meltzer ${ }^{2}$

${ }^{1}$ Department of Psychiatry, University Hospital of Maastricht, Maastricht, the Netherlands; ${ }^{2}$ Department of Psychiatry, Vanderbilt University, Nashville, USA; ${ }^{3}$ Psychiatric Hospital, University 'La Sapienza', Rome, Italy; ${ }^{4}$ Department of Hepatology, I Medical Clinic, University 'La Sapienza', Rome, Italy

Keywords: interferon; cytokines; depression; anxiety; peptidases; dipeptidyl peptidase IV

We have shown that treatment with interleukin-2 (IL-2) or interferon- $\alpha$ (IFN $\alpha$ ) may induce depressive symptoms and activation of the cytokine network and that IL-2 treatment may diminish serum dipeptidyl pepdidase IV (DPP IV) activity. ${ }^{1-3}$ DPP IV (EC 3.4.14.5) is a membran bound serine protease which catalyzes the cleavage of some cytokines and neuroactive peptides which modulate $T$ cell activity. ${ }^{4}$ The aims of the present study were to examine the effects of IFNa-based immunotherapy on serum DPP IV activity in relation to induction of the inflammatory response system. In 18 patients with chronic active hepatitis C, we determined the Montgomery and Asberg Rating Scale (MADRS), ${ }^{5}$ the Hamilton Anxiety Rating Scale (HAM-A), ${ }^{6}$ serum DPP IV activity, the kynurenine/tryptophan (K/T) quotient, which is an indicator of cytokine (in particular IFN)-induced catabolism of tryptophan, ${ }^{7}$ and serum interleukin-8 (IL-8) before starting therapy and 2, 4, 16 and 24 weeks after immunotherapy with IFN $\alpha$. IFNo-immunotherapy sig nificantly suppressed serum DPP IV 2-4 weeks and 1624 weeks after starting IFN $\alpha$-based immunotherapy. The reduction in serum DPP IV activity was more pronounced 16-24 weeks after starting immunotherapy than after 2-4 weeks. The IFNo-induced suppression of serum DPP IV activity was significantly correlated to IFN $\alpha$-induced increases in the MADRS and HAM-A and increases in the $\mathrm{K} / \mathrm{T}$ quotient and serum IL-8. In conclusion, long-term immunotherapy with IFN $\alpha$ suppresses serum DPP IV activity and the immunotherapyinduced changes in DPP IV are related to increases in severity of depression, anxiety and activation of the inflammatory response system. Molecular Psychiatry (2001) 6, 475-480

Immunotherapy with interleukin-2 (IL-2) and interferon- $\alpha$ (IFN $\alpha$ ) has become standard therapy in (selected) patients with hepatitis or kidney carcinoma. Immunotherapy with $\mathrm{IL}-2$ (with and without IFN $\alpha$ ) frequently induces depressive symptoms and full blown major depression within the first few days after starting treatment. ${ }^{1}$ IL-2 based immunotherapy induces the cytokine network, ${ }^{1}$ eg it elevates serum interleukin-6
(IL-6) and soluble IL-2 receptor (IL-2R) concentrations. ${ }^{1}$ Activation of the inflammatory response system (IRS) and administration of proinflammatory cytokines, such as IL-1 and IL-6, may induce depressogenic effects in experimental animals and humans. ${ }^{8-11}$ For example, in mice, sustained increases in serum IL-6 induces a rapid decline in preference for sucrose. ${ }^{11}$ Therefore, we have hypothesized that IRS activation could play a role in the etiology of $\mathrm{LL}-2$-induced depression. ${ }^{1}$

Long-term IFN $\alpha$-based immunotherapy (several weeks) induces depression in a considerable number of patients. $^{2,3}$ The IFN $\alpha$-induced increases in depression ratings are significantly related to IFN $\alpha$-induced changes in the serotonergic system, such as reductions in serum serotonin (5-HT) and plasma tryptophan, the precursor of 5-HT, and increases in serum kynurenine, the major tryptophan metabolite. ${ }^{2}$ The etiopathology of 'endogenous' (non-organic) depression may in part be related to disorders in the peripheral and central metabolism of 5-HT, such as lowered activity of the central presynaptic serotonergic neurons, a lowered availability of plasma tryptophan, and to changes in postsynaptic serotonergic receptors. ${ }^{12}$ Therefore, we have suggested that the IFN $\alpha$-based immunotherapyinduced changes in the serotonergic system may play a role in the development of IFN $\alpha$-induced depressive symptoms. ${ }^{2}$ Changes in other neurotransmitters, however, are undoubtedly involved in the etiopathology of depression. 'Endogenous' depression is also characterized by IRS activation as indicated by increased production of pro-inflammatory cytokines, such as $I L-1 \beta$, IL-6 and IFN $\gamma .^{8}$ Changes in the serotonergic system in 'endogenous' depression, such as reduced plasma tryptophan, are related to signs of IRS activation. ${ }^{8,13}$

'Endogenous' and IL-2-induced depression and conditions characterized by IRS activation, such as inflammatory and autoimmune disorders (rheumatoid arthritis, autoimmune chronic hepatitis and systemic lupus erythematosus) are accompanied by lowered serum activity of dipeptidyl peptidase IV (DPP IV; EC 3.4.14.5). ${ }^{14-16}$ DPP IV is a membrane-bound serine pro- 
tease that catalyses the cleavage of dipeptides from the amino-terminus of oligo- and polypeptides under definite structural conditions. ${ }^{4}$ On the T cell surface, DPP IV has proven to be identical with the CD26 molecule and its expression is increased-together with that of other activation markers. ${ }^{17}$ A suppressed activity of DPP IV may have psychoneuroimmune consequences, since some cytokines, chemokines and neuroactive peptides are potential substrates of DPP IV, eg IL-1 $\beta$, IL-6, substance $P$, growth hormone releasing hormone, neuropeptide $\mathrm{Y}$, and peptide YY. $.^{18,19} \mathrm{IL}-2$ based immunotherapy for 3-5 days significantly suppresses serum DPP IV activity. ${ }^{1}$

The aims of the present study are to examine whether: (1) therapy with IFN $\alpha$ in patients with hepatitis C suppresses serum DPP IV activity; and (2) the immunotherapy-induced reductions in serum DPP IV activity are inversely related to the development of depressive symptomatology and to signs of IRS activation.

Table 1 shows the MADRS, HAM-A and serum DPP IV values in baseline conditions and during IFN $\alpha$ based immunotherapy. Figure 1 shows the MADRS scores in the hepatitis-C patients both before and during therapy with IFN $\alpha$. Patients are divided into those with increases of more than 9 points on the MADRS from baseline to 4-6 months later and those without such an increase. Repeated measurement (RM) design analysis of variance (ANOVA) showed a significant effect of time $(\mathrm{F}=7.5, \mathrm{df}=1 / 16, P=0.04)$ and a significant time $\times$ MADRS status interaction $(\mathrm{F}=7.4, \mathrm{df}=$ $1 / 16, P=0.01$ ). Analyses of simple effects showed: (1) significant effects of time in patients who showed an increase of more than 9 points in the MADRS from baseline to 4-6 months later ( $\mathrm{F}=9.9, P=0.001)$, but not in those without such an increase $(\mathrm{F}=2.0, P=0.2)$; and (2) a significantly higher MADRS score in patients who showed an increase in the MADRS than in those without, 4-6 months $(F=10.4, P=0.003)$ but not $2-4$

Table 1 Effects of interferon-alpha immunotherapy on the Montgomery Asberg Depression Rating Scale (MADRS), the Hamilton-Anxiety (HAM-A) scale, serum dipeptidyl peptidase IV (DPP IV) activity, interleukin-8 (IL-8), and the kynurenine/tryptophan $(\mathrm{K} / \mathrm{T})$ quotient in 14 patients with hepatitis C

\begin{tabular}{|c|c|c|c|}
\hline Variables & Baseline & $2-4$ weeks & 4-6 months \\
\hline MADRS & $\begin{array}{c}4.7 \\
(3.2)\end{array}$ & $\begin{array}{l}10.5 \\
(9.4)\end{array}$ & $\begin{array}{l}10.0 \\
(7.7)\end{array}$ \\
\hline HAM-A & $\begin{array}{c}6.0 \\
(5.0)\end{array}$ & $\begin{array}{c}9.9 \\
(6.9)\end{array}$ & $\begin{array}{c}9.9 \\
(6.6)\end{array}$ \\
\hline DPP IV $\left(\mathrm{U} \mathrm{l}^{-1}\right)$ & $\begin{array}{l}42.9 \\
(9.5)\end{array}$ & $\begin{array}{l}33.6 \\
(7.1)\end{array}$ & $\begin{array}{l}28.8 \\
(7.8)\end{array}$ \\
\hline $\mathrm{IL}-8(\mathrm{pg} \mathrm{ml}-1)$ & $\begin{array}{c}17.9 \\
(14.7)\end{array}$ & $\begin{array}{c}28.8 \\
(13.2)\end{array}$ & $\begin{array}{c}21.7 \\
(10.5)\end{array}$ \\
\hline $\mathrm{K} / \mathrm{T}$ quotient & $\begin{array}{c}0.039 \\
(0.014)\end{array}$ & $\begin{array}{c}0.051 \\
(0.025)\end{array}$ & $\begin{array}{c}0.064 \\
(0.032)\end{array}$ \\
\hline
\end{tabular}

All data are shown as mean (SD).

See Results section for statistical analyses.

Molecular Psychiatry

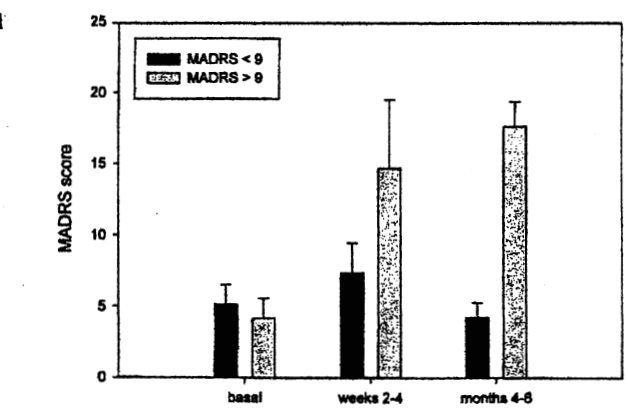

b

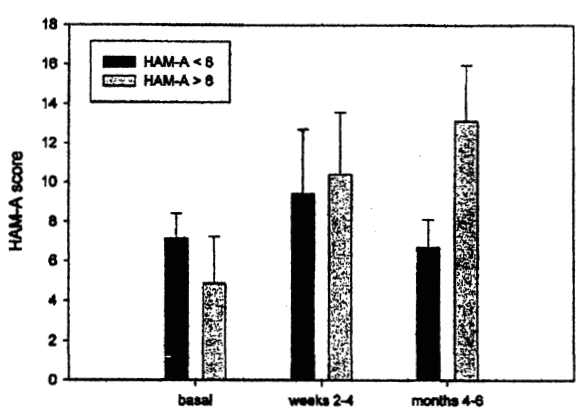

C

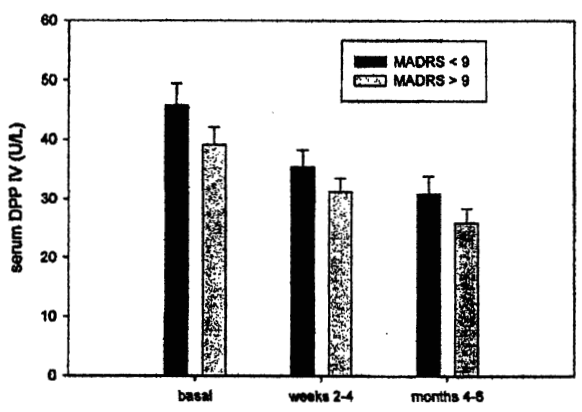

Figure 1 Effects of interferon-alpha immunotherapy on (a) the Montgomery Asberg Depression Rating Scale (MADRS), (b) the Hamilton-Anxiety (HAM-A) scale and (c) serum dipeptidyl peptidase IV (DPP IV) activity in 14 patients with hepatitis $C$, in baseline conditions (basal), and 2-4 weeks and 46 months after starting immunotherapy. The patients were divided into those whose MADRS scores increased more than 9 points from baseline to endpoint (MADRS $>9$ ) and those whose MADRS scores remained low during treatment (MADRS < 9) or into those whose HAM-A scores increased more than 6 points from baseline to endpoint (HAM-A > 6) and those whose MADRS scores remained low during treatment (HAM-A < 6). 
weeks after starting treatment $(\mathrm{F}=0.7, P=0.6)$ and not in the baseline condition $(\mathrm{F}=0.8, P=0.6)$. Figure 1 shows the HAM-A score in patients with an increase of more than 6 points on the HAM-A from baseline to 4-6 months later and in patients without such an increase. RM design ANOVA showed a significant effect of time $(\mathrm{F}=4.7 \mathrm{df}=1 / 14, P=0.04)$ and a significant time $\times$ group interaction $(F=3.5, \mathrm{df}=1 / 14, P=$ 0.05). Analyses of simple effects showed a significant effect of IFN $\alpha$ on the HAM-A score in patients with an increase of more than 6 points on the HAM-A $(F=8.2$, $P=0.002$ ), but not in those without such an increase $(\mathrm{F}=0.9, P=0.6)$. Dunn tests showed significantly higher HAM-A scores $2-4$ weeks $(t=2.66, P=0.01)$ and 4-6 months ( $t=2.66, P=0.01$ ) after starting IFN $\alpha$ based immunotherapy than in baseline conditions.

Figure 1 shows serum DPP IV values in hepatitis C patients, divided in patients who showed or did not show an increase of more than 9 points in the MADRS from baseline to 4-6 months later. ANOVA showed a significantly lower serum DPP IV activity in patients who showed an increase of more than 9 points in the MADRS than in those without such changes $(F=4.5$, $\mathrm{df}=1 / 36, P=0.039$ ). $\mathrm{RM}$ design ANOVA performed on the DPP IV values with the MADRS status as group factor, showed a significant effect of time $(F=42.7, \mathrm{df}$ $=2 / 21, P<10^{-4}$ ) but no significant time $\times$ group interaction $(F=0.3$, df $=2 / 21, P=0.7)$. Analyses on simple effects showed a significant effect of time in patients who showed an increase of more than 9 points in the MADRS $\left(\mathrm{F}=18.4, \mathrm{df}=2 / 24, P<10^{-4}\right)$ and in those who did not $\left(\mathrm{F}=24.6, \mathrm{df}=2 / 24, P<10^{-4}\right)$. Dunn tests showed significantly lower serum DPP IV values $2-4$ weeks $\left(t=5.98, P<10^{-4}\right)$ and 4-6 months $(t=9.20$, $P<10^{-4}$ ) after starting IFN $\alpha$ therapy than in baseline conditions and significantly lower serum DPP IV values 4-6 months after starting treatment than after $2-4$ weeks $(t=3.23, P=0.004)$. A RM design ANOVA with DPP IV as dependent variable and HAM-A status as grouping variable showed a significant effect of time $\left(\mathrm{F}=63.3, \mathrm{df}=2 / 23, P<10^{-4}\right)$ and a trend toward a significant time $\times$ group interaction $(F=3.0, d f=2 / 23$, $P=0.07)$. ANOVA with gender as factor showed significantly lower serum DPP IV activity in women (mean $=31.2 \pm 6.6 \mathrm{U} \mathrm{l}^{-1}$ ) than in men (mean $=38.0 \pm$ $11.1 \mathrm{U} \mathrm{l}^{-1}$ ). RM design ANOVA with gender as factor showed no significant gender $\times$ time interaction $(\mathrm{F}=$ 0.3 , df $=2 / 21, P=0.7$ ). Analysis on simple effects showed significant suppressant effects of immunotherapy with IFN $\alpha$ on serum DPP IV in women (F $=18.3$, $\left.\mathrm{df}=2 / 24, P<10^{-4}\right)$ and in men $(\mathrm{F}=24.5, \mathrm{df}=2 / 24, P$ $<10^{-4}$ ). There were no significant correlations between age and serum DPP IV at baseline $(r=-0.38, P=0.2)$, $2-4$ weeks after starting treatment $(r=-0.36, P=0.2)$ and 4-6 weeks later $(r=-0.32, P=0.3)$

Regression analyses, pooled over the subjects (intraclass correlations) and taking into account the three time points, showed significant time-relationships between the changes over time between serum DPP IV and the MADRS $(r=-0.47, F=7.7, \mathrm{df}=1 / 27, P=0.009)$ and the HAM-A score $(r=-0.52, \mathrm{~F}=10.1, \mathrm{df}=1 / 27, P$
$=0.004$ ). Figure 1, however, shows that serum DPP IV activity decreases in patients who have or have not increases in their MADRS scores of more than 9 points from baseline to endpoint. This suggests that the depression scores and DPP IV activity may be independent of one another. However, also using change scores, ie the $\Delta$ values = endpoint (4-6 months) minus the baseline values, we found a significant and inverse relationship between the $\Delta$ MADRS and $\Delta$ DPP IV values $(r=-0.76, P=0.003, n=14$; intra-class correlation pooled over the two patient groups divided according to their MADRS responses to IFN $\alpha$-based immunotherapy).

In order to examine the relationships between the changes over time in serum DPP IV and IRS activation we examined the correlations between the changes over time in serum DPP IV, on the one hand, and serum $\mathrm{IL}-8$ and the K/T quotient, on the other (intra-class correlations pooled over the subjects). Table 1 shows the IL-8 and the K/T values. RM design ANOVA performed on the IL-8 values showed a significant effect of time $(\mathrm{F}=5.2, \mathrm{df}=2 / 21, P=0.01$ ). Dunn tests showed significantly higher serum $\mathrm{L}-8 \quad 2-4$ weeks after starting immunotherapy than at baseline $(t=3.70, P=0.004)$ and no significant differences between baseline and 46 months later $(t=1.12, P=0.2)$. Regression analyses pooled over the subjects (intra-class correlation) showed significant time-relationships between the IFN $\alpha$-induced changes in serum DPP IV and IL-8 $(r=$ $-0.42, P=0.02)$. RM design ANOVA showed a significant enhancing effect of IFN $\alpha$-based immunotherapy on the $\mathrm{K} / \mathrm{T}$ ratio $\left(\mathrm{F}=41.3, \mathrm{df}=2 / 19, P<10^{-4}\right)$, but no significant time $\times$ MADRS status effect $(F=1.4, \mathrm{df}=$ $2 / 19, P=0.3$ ). Dunn tests showed a significantly higher $\mathrm{K} / \mathrm{T}$ quotient $2-4$ weeks $\left(t=5.54, P<10^{-4}\right.$ and $4-6$ months $\left(t=9.11, P<10^{-4}\right)$ after starting IFN $\alpha$ treatment and a significantly higher $\mathrm{K} / \mathrm{T}$ quotient 4-6 months after treatment than at $2-4$ weeks $(t=3.58, P=0.002)$. There was a significant negative correlation between the changes over time in serum DPP IV and the K/T quotient $\left(r=-0.75, \mathrm{~F}=34.3, \mathrm{df}=1 / 27, P<10^{-4}\right.$; regression analysis pooled over the subjects).

To the best of our knowledge, this is a first study reporting that long-term ( 2 weeks-6 months) IFN $\alpha$ based immunotherapy significantly suppresses serum DPP IV activity in patients with hepatitis C. Previously, we have shown that treatment with IL-2 (with or without IFN $\alpha$ ), but not IFN $\alpha$ alone, for 3-5 days significantly suppressed serum DPP IV activity. ${ }^{1}$ Thus, it appears that short-term immunotherapy with IFN $\alpha$ ( $<$ 5 days) does not affect DDP IV activity, whereas longer term therapy ( $>2$ weeks) results in significant effects. The mechanisms whereby IFN $\alpha$-based immunotherapy may decrease serum DPP IV activity have remained elusive. It has been shown that IFN $\alpha$ may increase DPP IV/CD26 mRNA expression in renal tubular epithelial cells and renal cell carcinoma cells in culture. ${ }^{20}$ Other cytokines, however, whose production may be induced by IFNs, such as tumor necrosis factor- $\alpha$ (TNF $\alpha$ ) and TGF $\beta$, may reduce DPP IV mRNA and enzyme activity. ${ }^{20,21}$ 
A second major finding of this study is the significant and inverse relationship between the IFN $\alpha$-induced increases in the MADRS and HAM-A scores and the decreases in DPP IV activity and the significantly lower serum DPP IV activity in patients who have a marked increase in the MADRS as compared to those without such an increase. These findings extend those of previous papers showing that 'endogenous' major depression is accompanied by lowered serum DPP IV activity and that the IL-2-induced decreases in serum DPP IV are significantly and inversely correlated with the increases in the MADRS. ${ }^{1}$ IFN $\alpha$-based immunotherapy is known to induce depressive and anxiety symptoms and full blown major depression in a number of patients. ${ }^{2,3}$ IFN $\alpha$-based immunotherapy has depressogenic properties through different mechanisms. First, it induces the production of pro-inflammatory cytokines, such as IL-6, which have depressogenic activities. Second, IFN $\alpha$ affects the serotonergic system and increases 5-HT transporter mRNA and uptake activity;22 suppresses 5-HT concentrations in brain or serum; ${ }^{23}$ and induces the catabolism of tryptophan to kynurenine, probably through induction of the IDO pathway by interferons. ${ }^{24}$ Third, activation of the hypothalamic-pituitary-adrenal (HPA) axis is a common feature of depression ${ }^{13}$ and occurs following IFN $\alpha$-based immunotherapy. ${ }^{25}$ Previously, it was shown that circulating IL-6 increases after systemic IFN $\alpha$ administration, resulting in activation of the HPA-axis. ${ }^{25}$ In the present study, however, we did not measure the HPAaxis hormones.

The third major finding of this study is that the IFN $\alpha$ induced decrements in serum DPP IV activity are significantly and inversely related to indicants of IFN $\alpha$ induced IRS activation, ie increased serum IL-8 and $\mathrm{K} / \mathrm{T}$ quotient. IL-8 is produced by monocytes, macrophages, neutrophils and endothelial cells after stimulation by IL- 1 and TNF $\alpha$ and it induces the chemotaxic activity of immune cells. ${ }^{26}$ An increased $\mathrm{K} / \mathrm{T}$ quotient indicates an increased catabolism, of tryptophan probably through IFN $\alpha$-induced IDO. IFN $\gamma$ and IFN $\alpha$ may induce IDO in the brain, kidney, lung, spleen, duodenum and immune cells. ${ }^{24,27}$ Our findings extend previous results showing that inflammatory conditions and disorders are accompanied by lower serum DPP IV activity ${ }^{14-16,28}$ and that in depression lowered serum DPP IV is related to signs of IRS activation. ${ }^{28}$

\section{Materials and methods}

Subjects

Eighteen chronic active hepatitis $C$ patients participated in this study. There were 10 men and 8 women with a mean age $( \pm S D)$ of $40.5( \pm 11.5)$ years. The patients were recruited at the Hepatology Department, I Medical Clinic at the University of Rome 'La Sapienza' between November 1997 and May 1998. Three of the patients stopped treatment after a few days: one due to a reduction in the number of platelets and two because of IFN $\alpha$ low compliance. In one patient, the baseline measurements of serum DPP IV and plasma IL-8 were missing. Finally, 14 hepatitis $C$ patients completed the IFN $\alpha$ treatment, the psychiatric assessments and the blood samplings, ie eight men and six women with a mean age $( \pm S D)$ of $41.7( \pm 10.8)$ years. Inclusion criteria were: (1) a diagnosis of chronic active hepatitis $C$, which was based on persistently elevated transminase values (1.5 over the normal range for at least 6 months), anti-HCV positivity and histological report; (2) age $<65$ years; (3) histological diagnosis of chronic hepatitis; (4) positivity for anti-HCV antibodies and HCV RNA in serum; and (5) platelet counts $>100.000 \mathrm{~mm}^{-3}$. The exclusion criteria for patients were: (1) patients with liver cirrhosis, heart, kidney or autoimmune diseases; (2) infection with HBV, HDV or HIV; (3) WBC counts $<4000 \mathrm{~mm}^{-3}$; (4) a PT $<60 \%$; (5) chronic alcohol abuse or active intravenous drug use (IVDU); and (6) patients with a past or present history of axis-I disorders, such as major and bipolar depression. None of the patients was taking any psychotropic drugs for at least 1 year prior to IFN $\alpha$-based immunotherapy. None of the patients took any other medication besides IFN $\alpha$, including antidepressants, during the study period. No changes in physical status were recorded during the treatment period. Illness onset and awareness ranged from a minimum of 1 to a maximum of 2 years. The local ethical committee approved the study design and the subjects gave written informed consent after the study protocol was fully explained.

\section{Methods}

The hepatitis $C$ patients were randomized to receive two IFN 22 (Roferon, Roche, Switzerland) treatment schedules: the first with $6 \mathrm{MU}$ IFN $\alpha$ s.c. per day, 6 days a week for 4 weeks; the second with 6 MU IFN s.c. three times a week for 4 weeks. There were no significant differences at any of the time points in the clinical ratings, serum DPP IV activity, serum IL-8 or the K/T quotient between these two time points. Therefore, subsequent statistical analyses were performed on both treatment groups combined. After the initial 4-week treatment period, all patients continued IFN $\alpha$ therapy with $6 \mathrm{MU}$ IFN s.c. three times a week for 12 weeks. Responders at 4 months received 3 MU IFN s.c. three times a week for a further 8 months. After an overnight fast, plasma or serum for the assay of DPP IV activity, IL-8, kynurenine, and tryptophan were collected at $0800 \mathrm{~h}$ at baseline (before starting treatment) and 2, 4, 16 , and 24 weeks after starting the immunotherapy. During each of these sessions, patients completed the Montgomery Asberg Depression Rating Scale (MADRS) $^{5}$ and the Hamilton Anxiety Rating Scale. ${ }^{6}$

DPP IV activity was determined in serum by means of a colorimetric method using the chromogenic substrate glycyl-L-proline-p-nitroanilide tosylate (Gly-Pro$p$-NA). ${ }^{29}$ We adapted this method for direct continuous measurement on the centrifugal analyzer Cobas Bio (Roche Diagnostics, Brussels, Belgium). The sensitivity of the assay was $3.0 \mathrm{U} \mathrm{l}^{-1}$. The analytical imprecision (ie the coefficient of variation, expressed as a percentage, $\mathrm{CV} \%$ ) of the DPP IV assay was CV $=2.2 \%$. Serum IL-8 was measured by a quantitative enzyme-linked

Molecular Psychiatry 
immunosorbent assay (ELISA) (Eurogenetics, Tessenderlo, Belgium) based on appropriate and validated sets of monoclonal antibodies. The sensitivity of the assay was $2.0 \mathrm{pg} \mathrm{ml}^{-1}$ and the intra-assay $\mathrm{CV}$ value was less than $8 \%$. Plasma tryptophan was measured by means of an HPLC as explained by us. ${ }^{13}$ The intra-assay CV value obtained in our laboratory was $3.3 \%$. Serum kynurenine was determined by means of HPLC as described by Holmes. ${ }^{30}$ The intra-assay CV value for kynurenine was $4.5 \%$. The kynurenine per tryptophan quotient $(\mathrm{K} / \mathrm{T})$ was computed. The latter estimates the activity of indoleamine 2,3 dioxygenase (IDO), the major tryptophan catabolizing enzyme, which is inducible by interferons and $I L-2{ }^{7}$ In order to minimize the analytical variability in the results, all blood specimens for the assays of the above parameters were performed in a single run with a single lot number of reagents and consumables employed by a single operator.

\section{Statistics}

Correlations between variables were ascertained by means of Pearson's product moment correlation coefficients. Regression analyses, which are pooled over the subjects (intra-class correlations) and which take in account the three time points, were used in order to assess time-relationships between variables. This method eliminates the inter-individual variability and assesses the relationships between the changes over time in two sets of variables. Group mean differences were checked with ANOVA. RM design ANOVAs were performed on the baseline values and the values obtained during IFN $\alpha$ immunotherapy. First we used the RM ANOVA to check the overall effects of IFN $\alpha$ on serum DPP IV and the other variables (results not shown). There were no significant differences in any of the variables either between weeks 2 and 4 or between months 4 and 6 . In order to check the rapid (first month) vs protracted (several months) effects of IFN $\alpha$ based immunotherapy, we employed the nadir (DPP IV) or peak (MADRS, HAM-A, IL-8, K/T quotient) values obtained at weeks 2 and 4 , and the nadir/peak values obtained at months 4 and 6 for those variables which significantly increased and decreased, respectively, during IFN $\alpha$-based immunotherapy. The results of RM design ANOVAs were corrected for sphericity. For the MADRS and HAM-A scores and DPP IV values, we examined: (i) the within-subject variability with the baseline and two treatment conditions as time factor, and (ii) two-way interactions between time $\times$ MADRS/HAM-A status. $A$ priori comparisons between treatment means were ascertained with the Dunn test. Tests on simple effects were carried out in order to examine significant main effects or significant interaction patterns.

\section{Acknowledgements}

This work was supported by a grant from the National Council for Research No. 203.04.17 of 09/06/97. We would like to thank Professor Dr S Scharpe, Dr DeMeester and C Durinx for their valuable help.

\section{References}

1 Maes M, Capuron L, Ravaud A, Gualde N, Bosmans E, Bonaccorso $S$ et al. Immunochemotherapy with interleukin-2 and/or interferon-alpha in cancer patients induces lowered serum dipeptidyl peptidase IV activity, which is related to therapy-induced depressive symptoms and immune activation. Neuropsychopharmacology 2000; 24: 130-140.

2 Bonaccorso S, Meltzer HY, Maes M. Psychological and behavioral effects of interferons. Curr Opinion Psychiatry 2000; 13: 637-677. 3 Bonaccorso S, Marino V, Biondi M, Grimaldi F, Ippoliti F, Maes $\mathrm{M}$. Major depression induced by interferon-alpha in patients affected by hepatitis C virus. J Affect Disord 2001; (in press).

ted by hepatitis C virus. J Affect Disord 2001; (in press).
4 De Meester I, Korom S, Van Damme J, Scharpe S. CD26, let it cut 4 De Meester I, Korom S, Van Damme J, Scharpe S. C
or cut it down. Immunol Today 1999; 20: 367-375,

5 Montgomery SA, Asberg A. A new depression scale designed to be sensitive to change. Br J Psychiatry 1979; 134: 382-389.

6 Hamilton $\mathrm{M}$. The assessment of anxiety by rating. $\mathrm{Br} J P_{\text {sychiatry }}$ 1959; 32: 50-55.

7 Widner B, Sepp N, Kowald E, Ortner U, Wirleitner B, Fritsch P et al. Enhanced tryptophan degradation in systemic lupus erythematosus. Immunobiology 2000; 201: 621-630.

8 Maes M. Major depression and activation of the inflammatory response system. Adv Exp Med Biol 1999; 461: 25-46.

9 Yirmiya R. Endotoxin produces a depressive-like episode in rats. Brain Res 1996; 711: 163-174.

10 Bluthé RM, Crestani F, Kelley KW, Dantzer R. Mechanisms of the behavioral effects of interleukin 1. Ann NY Acad Sci 1992; 650: 268-275.

11 Sakic B, Szechtman H, Braciak T, Richards C, Gauldie J, Denburg JA. Reduced preference for sucrose in autoimmune mice: a possible role of interleukin-6. Brain Res Bull 1997; 44: 155-165.

12 Maes M, Meltzer HYM. The serotonin hypothesis of major depression. In: Bloom FE, Kupfer DJ (eds). Psychopharmacology the Fourth Generation of Progress. Raven Press: New York, 1995, pp 933-944.

13 Maes M, Wauters A, Verkerk R, Neels H, vanGastel A, Cosyns P et al. Lower L-tryptophan availability in depression: a marker of a more generalized disorder in protein metabolism. Neuropsychopharmacology 1996; 15: 243-251.

14 Maes M, De Meester I, Vanhoof G, Scharpé S, Bosmans E, Vandervorst $\mathrm{C}$ et al. Decreased serum dipeptidyl peptidase IV activity in major depression. Biol Psychiatry 1991; 30: 577-586.

15 Elgun S, Keskinege A, Kumbasar H. Dipeptidyl peptidase IV and adenosine deaminase activity. Decrease in depression. Psychoneuroendocrinology 1999; 24: 823-832.

16 De Meester IAJ. Characterization of human lymphocytic dipeptidyl peptidase IV and its identification as the activation antigen CD 26. PhD Thesis, Antwerp, 1992

17 Durinx C, Lambeir AM, Bosmans E, Falmagne JB, Berghmans R, Haemers $\mathrm{A}$ et al. Molecular characterization of dipeptidyl peptidase activity in serum soluble CD26/dipeptidyl peptidase IV is responsible for the release of X-Pro dipeptides. Eur J Biochem 2000; 267: 5608-5613.

18 De Meester I, Durinx C, Bal G, Proost P, Struyf S, Goossens F et al. Natural substrates of dipeptidyl peptidase IV. In: Langner J, Ansorge $S$ (eds). Cellular Peptidases in Immune Functions and DisAnsorge $S$ (eds). Cellular Peptidases in Immune Functions
eases 2. Plenum Publishers: New York, 2000, pp 67-87.

19 Van Hoof G, Goossens F, De Meester I, Hendriks D, Scharpé S. Van Hoof G, Goossens F, De Meester I, Hendriks D, Scharpé S.
Proline motifs in peptides and their biological processing. FASEB 1995; 9: 736-744.

20 Kehlen A, Gohring B, Langer J, Riemann D. Regulation of the expression of aminopeptidase $A$, aminopeptidase $\mathrm{N} / \mathrm{CD} 13$ and dipeptidylpeptidase IV/CD 26 in renal carcinoma cells and renal tubular epitelial cells by cytokines and CAMP-increasing mediators. Clin Exp Immunol 1998; 111: 435-441.

21 Riemann D, Kehlen A, Langner J. Stimulation of the expression and the enzyme activity of aminopeptidase N/CD 13 and dipeptidylpeptidase IV/CD 26 on human renal cell carcinoma cells and renal tubular epithelial cells by $T$ cell-derived cytokines, such as $\Pi-4$ and IL-13. Clin Exp Immunol 1995; 100: 277-283.

22 Morikawa O, Sakei N, Obara $H$ Saito N. Effocts of intefomolpha Morikawa O, Sakai N, Obara H, Saito N. Effects of interferon-alpha, the serotonin transporter. Eur I Pharmacol 1998; 349: 317-324. 
(1)

\section{IFN $\alpha$ treatment of hepatitis $C$ patients}

23 Kamata M, Higuchi H, Yoshimoto M, Yoshida K, Shimizu T. Effect of single intracerebroventricular injection of alpha-interferon on mono acol 2000; 10: 129-132.

24 Taylor MW, Feng G. Relationship between interferon-gamma, indolamine 2,3-dioxygenase, and tryptophan catabolism. FASEB J 1991; lamine 2,3-dioxy

25 Shimizu $\mathrm{H}$, Ohtani $K$, Sato $N$, Nagamine $T$, Mori $M$. Increase in serum interleukin-6, plasma ACTH and serum cortisol levels after systemic interferon-alpha administration. Endocrinol J 1995; 42: systemic interferon-alpha administration. Endocrinol $J$ 1995; 42: 551-556.

Masson: Paris, 1996.

27 Recht M, Borden EC, Knight E. A human 15-kDa IFN-induced protein induces the secretion of IFN-gamma. I Immunol 1991; 147: 2617-2623.

28 Maes M, DeMeester I, Verkerk R, Demedts P, Wauters A, Vanhoof
G et al. Dipeptidyl peptidase serum activity in treatment resistant depression: relationships with immune-inflammatory markers. Psychoneuroendocrinology. 1997; 22: 65-78.

29 Nagatsu T, Hino M, Fuyamada H, Hayakawa T, Sakibara S, Nakagawa $\mathrm{Y}$ et al. New chromogenic substrates for X-Pro dipeptidyl aminopeptidase. Anal Biochem 1976; 74: 466-476.

30 Holmes EW. Determination of serum kynurenine and hepatic tryptophan dioxygenase activity by high-performance liquid chromatography. Anal Biochem 1988; 172: 518-525.

Correspondence: M Maes, MD, PhD, Professor of Psychiatry, ChairCorrespondence: $\mathrm{M}$ Maes, $\mathrm{MD}$, $\mathrm{Ph}$, Professor of Psychiatry, Chairman Department of Psychiatry \& Neuropsychology, University Hospital of Maastricht, Postbus 5

Received 12 September 2000; revised 2 January 2001; accepted 4 January 2001

Molecular Psychiatry 


\section{Chapter 8}

\section{Lower activities of serum peptidases predict higher depressive and anxiety levels following interferon-alpha-based immunotherapy in patients with hepatitis C}

Maes M, Bonaccorso S. Lower activities of serum peptidases predict higher depressive and anxiety levels following interferon-alpha-based immunotherapy in patients with hepatitis C.

Acta Psychiatr Scand 2004: 109: 126-131. (c) Blackwell Munksgaard 2004.

Objective: There is evidence that in patients with chronic hepatitis C, immunotherapy with interferon-alpha (IFN $\alpha$ ) may induce depression. A lowered activity of peptidases, such as prolylendopeptidase (PEP) and dipeptidyl peptidase IV (DPP IV), occurs in depression. This study examines whether lowered serum PEP or DPP IV activity before starting IFN $\alpha$-based immunotherapy predicts the increase in depressive symptoms during immunotherapy.

Method: Serum PEP and DPP IV activities are measured in patients with hepatitis $C$ before and 2,4 and 16 weeks after starting IFN $\alpha$ based immunotherapy. The Montgomery Asberg Depression Rating Scale (MADRS) and the Hamilton Anxiety Rating Scale (HAM-A) are completed.

Results: Patients with lower baseline PEP or DPP IV had significantly higher MADRS and HAM-A scores both at baseline and during immunotherapy. Patients with lower baseline DPP IV had significantly higher increases in the MADRS following IFN $\alpha$ treatment.

Conclusion: Lower baseline PEP and DPP IV predict higher depressive and anxiety ratings during IFN $\alpha$-based immunotherapy.

\author{
M. Maes ${ }^{1,2}$, S. Bonaccorso $0^{1,3}$ \\ 'Department of Psychiatry, University of Maastricht, \\ Maastricht, The Netherlands, ${ }^{2}$ Clinical Research Center \\ for Mental Health, Limburg, Belgium, and ${ }^{3}$ University La \\ Sapienza, Rome, Italy
}

\section{Introduction}

There is now evidence that peptidases, such as prolylendopeptidase (PEP) and dipeptidyl peptidase IV (DPP IV), play a role in the pathophysiology of major depression (1-3). PEP (EC 3.4.21.26) is a cytosolic endopeptidase which degrades proteins such as arginine vasopresin (AVP), thyrotropin releasing hormone (TRH), substance P, oxytocin, and bradykinin (4). DPP IV (EC 3.4.14.5) is a membrane-bound peptidase, which degrades proteins, such as substance $P$, neuropeptide $\mathrm{Y}$ and peptide $\mathrm{YY}$ and maybe cytokines, such as interleukin-1 (IL-1) and IL-6 $(5,6)$. On the surface of T cells, DPP IV is identical with CD26 and plays a crucial role in $T$ cell activation and the production of cytokines, such as IL-2 and interferon- $\gamma$ (IFN $\gamma$ ) (7-9). We have hypothesized that a decreased activity of the above peptidases could be related to the pathophysiology of depression, because a lowered activity of these peptidases causes a decreased degradation of behaviorally active peptides, such as AVP, TRH and substane $P$, which play a role in depression, anxiety, emotions and social behavior $(3,10)$. Moreover, decreased serum activities of these peptidases may predict the occurrence of depression. For example, in parturient women, lowered serum PEP activity before delivery predicts postpartum depression (11).

There is also evidence that an increased production of pro-inflammatory cytokines, such as IL-1 $\beta$, IL-6 and IFN $\gamma$, is involved in the etiology of depression (12). Depression is accompanied by increased production of those cytokines and by an acute phase response, which is known to be induced by pro-inflammatory cytokines (12). Antidepressants have, in vivo and in vitro, anti-inflammatory 
effects by reducing the production of pro-inflammatory cytokines, e.g. IFN $\gamma$ and IL-6, and increasing that of anti-inflammatory cytokines, e.g. IL-10 $(13,14)$. Immune activation and the administration of pro-inflammatory cytokines, such as IL-6, may induce depressive symptoms and anxiety in experimental animals and humans (12). Immunotherapy of patients with kidney carcinoma or hepatitis C with cytokines, such as IFN $\alpha$, may induce depressive symptoms and full blown major depression in a considerable number of patients $(10,15)$. Moreover, treatment with IFN $\alpha$ significantly suppresses serum DPP IV activity $(10,16)$. The immunotherapy-induced decreases in serum DPP IV are significantly and inversely related to immunotherapy-induced increases in severity of depression (10, 16). No research, however, has examined whether lowered PEP or DPP IV activity determined before starting IFN $\alpha$-based immunotherapy may predict higher depression or anxiety ratings following treatment.

The aims of the present study were to examine whether lower baseline serum PEP or DPP IV activity may predict a greater increases in depressive and anxiety symptoms following IFN $\alpha$-based immunotherapy.

\section{Material and methods \\ Subjects}

Eight men and six women with a mean age $( \pm S D)$ of $41.8( \pm 11.1)$ years with chronic, active hepatitis $C$ participated in this study. We included patients with a diagnosis of chronic active hepatitis $\mathrm{C}$ based on persistently elevated transaminase values (1.5 over the normal range for at least 6 months), positivity for anti-HCV antibodies and $\mathrm{HCV}$ RNA in serum and a histological diagnosis of chronic hepatitis. Patients had to be $<65$-years old with a platelet count $>100000 / \mathrm{mm}^{3}$. We excluded patients with HBV, HDV or HIV infection, liver cirrhosis, heart, kidney or autoimmune diseases, and patients with a WBC count $<4000$ / $\mathrm{mm}^{3}$ and a PT $<60 \%$. We excluded patients with a past or present history of psychiatric disorders, including major and bipolar depression and alcohol abuse or active intravenous drug use. During the study period, patients did not take any medication other than IFN $\alpha$. Not one of the patients was taking psychotropic drugs for at least 1 year prior to IFN $\alpha$-based immunotherapy. We recorded no changes in the physical status during the treatment period. Illness onset and awareness ranged from a minimum of one to a maximum of 2 years. The study design was approved by the local ethical committee and the patients gave written informed consent after the study protocol was fully explained. Thirteen normal volunteers (seven men and six women with a mean age of $40.5 \pm 13.1$ years) were included to compare the baseline PEP and DPP IV activities in the patients with the normal control values. The normal volunteers were free of any medical illness and they had a negative past, present and family history of psychiatric disorders. Not one of the controls had ever been taken psychotropic drugs or drugs which affect the endocrine or immune systems. All were free of any drugs for at least 1 year and all were free of any inflammatory, infectious or allergic reactions for at least 2 weeks prior to the blood samplings.

Method

The patients with chronic, active hepatitis $\mathrm{C}$ were randomized to receive two IFN 2 (Roferon, Roche, Basel, Switzerland) treatment schedules: the first $6 \mathrm{MU}$ IFN $\alpha$ subcutaneously per day six times a week and the second group with $6 \mathrm{MU}$ IFN $\alpha$ subcutaneously three times a week. These treatments were continued for 4 weeks. After this initial 4-week treatment period, all patients received IFN $\alpha$ therapy with 6 MU IFN subcutaneously three times a week for another 12 weeks. There were no significant differences at any of the time points in the clinical ratings, serum PEP or DPP IV activity between these two treatments and, subsequently, the statistical analyses were performed on both treatment groups combined. Before starting treatment (baseline) and 2, 4, and 16 weeks after starting IFN $\alpha$-based immunotherapy, serum for the assay of PEP and DPP IV activity was collected at 08.00 hours after an overnight fast. During each of these sessions, patients completed the Montgomery Asberg Depression Rating Scale (MADRS) (17) and the Hamilton Anxiety Rating Scale (HAM-A) (18).

Serum PEP activity was determined by a fluorimetric method with the synthetic substrate Z-glycylprolyl-4-methylcoumarinyl-7-amide (Bachem Feinchemikalien AG, Bubendorf, Switserland) (19). The intra-assay coefficient of variation (CV) in our laboratory was $4.8 \%$. DPP IV activity was determined as described previously with a colorimetric method using the chromogenic substrate glycyl-L-proline-p-nitroanilide tosylate (Gly-Pro-p-NA) (20). The intra-assay CV value of the DPP IV assay was CV $=2.2 \%$. In order to minimize effects of analytical variability, all serum specimens were assayed in a single run with a single lot number of reagents and consumables 
employed by a single operator. Only the baseline values for PEP and PEP are presented in this study. The measurements of DPP IV at the different time points are presented somewhere else (16).

\section{Statistics}

Analysis of variance (ANOVA) is used to examine whether patients divided into groups with regard to their baseline serum PEP and DPP IV activity differ in their MADRS - HAM-A scores. Multiple comparisons among treatment means were checked with Fisher's least significant difference (LSD). Repeated measure (RM) design ANOVA (regression method) is employed to examine (i) the withinsubject variability (time effect) with the assessment of the effects of immunotherapy (baseline vs. posttreatment); and (ii) between-subject variability, with comparisons of the patients divided into groups; and (iii) two way interactions between time $\times$ groups. Analyses on simple effects are used to check the effects of time in the diagnostic groups separately. Regression analyses are used to examine relationships between variables.

\section{Results}

Figure 1 shows the MADRS scores in the 14 patients at the four time points. Four of the patients developed a major depression according to DSM-IV criteria. The patients were divided in two groups according to the median values of their baseline PEP (cut off value $\leq 0.240 \mathrm{U} / \mathrm{ml}$ ) and DPP IV (cut off value $\leq 38.9 \mathrm{U} / \mathrm{ml}$ ) values. Table 1 shows the MADRS and HAM-A values at the four time points in patients who, at baseline, were divided

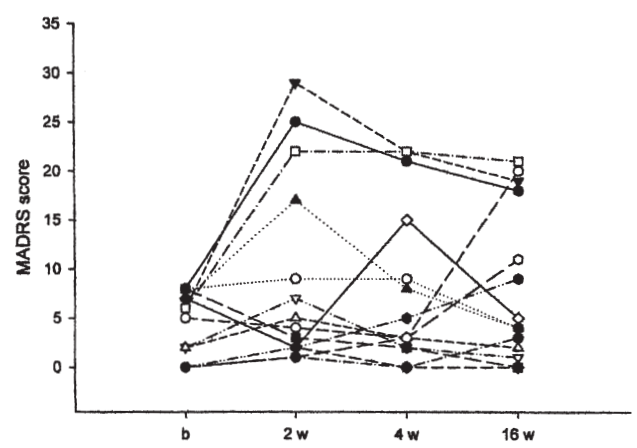

Fig. 1. Scatter plot of the MADRS scores in 14 patients with chronic, active hepatitis $\mathrm{C}$, before treatment (b) and during treatment with interferon-alpha, $2(2 \mathrm{w}), 4(4 \mathrm{w})$ and $16(16 \mathrm{w})$ weeks after starting treatment with interferon-alpha. into those with lower vs. higher serum PEP (cut off value $\leq 0.240 \mathrm{U} / \mathrm{ml}$ ) and DPP IV (cut off value $\leq 38.9 \mathrm{U} / \mathrm{ml}$ ) values. The MADRS scores were significantly higher at all time points in patients who had lower baseline serum PEP than in those with higher PEP values $(F=6.0, \mathrm{df}=1 / 48$, $P=0.02$; the mean values over the four time points are $10.1 \pm 7.5$ vs. $5.1 \pm 7.2$ ). The MADRS scores were significantly higher in patients who had lower baseline DPP IV than in those with higher DPP IV $(F=21.0, \mathrm{df}=1 / 48, P=0.0001$; the mean values over the four time points are $11.6 \pm 8.5$ vs. $3.6 \pm 3.8$ ). Patients with lower serum PEP had significantly higher HAM-A values as compared with those with higher PEP values $(F=12.1$, $\mathrm{df}=1 / 48, P=0.001$; the mean values over the four time points are $10.0 \pm 6.7 \mathrm{vs.}$ $4.8 \pm 4.7)$. Patients with lower DPP IV had significantly higher HAM-A values than those with higher baseline serum DPP IV $(F=9.9$, $\mathrm{df}=1 / 48, P=0.003$; the mean values over the four time points are $9.8 \pm 7.0$ vs. $5.0 \pm 4.4$ ).

Table 1 shows the peak MADRS and peak HAM-A scores in patients who were divided according to their baseline PEP or DPP IV values. We found significantly higher MADRS values in patients with lower serum PEP $(F=5.2, \mathrm{df}=1 / 12$, $P=0.039)$, a significant effect of time $(F=12.1$, $\mathrm{df}=1 / 12, P=0.005)$, but no significant interaction between time $\times$ group $(F=0.2, \mathrm{df}=1 / 12$, $P=0.7$; all results of RM design ANOVA). We detected significantly higher MADRS values in patients with lower serum DPP IV $(F=12.1$, $\mathrm{df}=1 / 12, P=0.005)$, a significant effect of time $(F=20.3, \mathrm{df}=1 / 12, P=0.0009)$ and a significant time $\times$ group interaction $(F=8.3, \mathrm{df}=1 / 12$, $P=0.01$; all results of RM design ANOVA). Post hoc analyses showed a significantly higher MADRS score in patients with a lower than in those with higher DPP IV activity $(F=20.4, \mathrm{df}=1 / 24$, $P=0.0003$ ), whereas no significant differences in baseline MADRS scores could be found between both groups $(F=0.5, \mathrm{df}=1 / 24, P=0.5)$. Post hoc analyses also showed significant effects of time in patients with lower serum DPP IV $(F=27.3$, df $=1 / 12, \quad P=0.0004)$, but not in those with higher serum DPP IV $(F=1.3, \mathrm{df}=1 / 12$, $P=0.3)$. RM design ANOVA performed on the HAM-A scores showed significantly higher HAM$A$ values in patients with lower vs. higher serum PEP $(F=6.6, \mathrm{df}=1 / 12, P=0.02)$, a significant effect of time $(F=15.9, \mathrm{df}=1 / 12 ; P=0.002)$, but no significant time $\times$ treatment interaction $(F=0.4, \mathrm{df}=1 / 12, P=0.6)$. There were no significant differences between patients with lower vs. higher serum DPP IV in the HAM-A scores 
Peptidases and cytokine-induced depression

Table 1. The mean Montgomery Asberg Depression Rating Scale (MADRS) and Hamilton Anxiety Rating Scale (HAM-A) values in baseline conditions and during treatment with interferon- $\alpha(2,4$ and 16 weeks after starting treatment) in patients with hepatitis $C$ who were divided according to the median split of their baseline prolylendapeptidase (PEP) and dipeptidyl peptidase IV (DPP IV) serum levels into those with lower vs. higher serum PEP (cut off value $\leq 0.240 \mathrm{U} / \mathrm{mll}$ ) and DPP IV (Cut off value $\leq 38.9 \mathrm{U} / \mathrm{ml}$ values.

\begin{tabular}{lllrrrr}
\hline Dependent variable & Groups & Baseline & 2 weeks & 4 weeks & 16 weeks & Peak \\
\hline MADRS & PEP $\leq 0.240 \mathrm{U} / \mathrm{ml}$ & $7.0(1.1)$ & $11.7(9.6)$ & $11.4(8.1)$ & $10.3(8.9)$ & $15.9(7.7)$ \\
& PEP $>0.240 \mathrm{U} / \mathrm{ml}$ & $2.4(2.9)$ & $6.7(10.1)$ & $5.0(7.7)$ & $6.4(6.9)$ & $9.4(9.2)$ \\
MADRS & DPP IV $\leq 38.9 \mathrm{U} / \mathrm{ml}$ & $5.7(2.7)$ & $13.1(11.8)$ & $13.6(8.6)$ & $14.0(7.3)$ & $18.7(7.4)$ \\
& DPP IV $>38.9 \mathrm{U} / \mathrm{ml}$ & $3.7(3.5)$ & $5.3(5.6)$ & $2.9(2.8)$ & $2.7(3.2)$ & $6.6(5.2)$ \\
GHAM-A & PEP $\leq 0.240 \mathrm{U} / \mathrm{ml}$ & $9.3(4.6)$ & $12.6(6.6)$ & $6.6(5.3)$ & $11.7(8.9)$ & $14.9(7.3)$ \\
& PEP $>0.240 \mathrm{U} / \mathrm{ml}$ & $2.7(2.7)$ & $7.0(6.8)$ & $2.9(3.6)$ & $6.8(3.7)$ & $10.3(4.7)$ \\
HAM-A & DPP IV $\leq 38.9 \mathrm{U} / \mathrm{ml}$ & $7.7(5.7)$ & $11.6(8.3)$ & $7.3(5.5)$ & $12.7(8.0)$ & $15.0(7.6)$ \\
& OPP N $>38.9 \mathrm{U} / \mathrm{ml}$ & $4.3(3.8)$ & $8.0(5.7)$ & $2.1(2.1)$ & $5.6(3.9)$ & $10.1(3.9)$ \\
\hline
\end{tabular}

All results are shown as mean $( \pm S D$

Peak: highest value obtained at weeks 2,4 or 16 .

( $F=2.9, \mathrm{df}=1 / 12, P=0.1$ ), a significant effect of time $(F=15.7, \mathrm{df}=1 / 12, P=0.01)$, but no significant time $\times$ treatment interaction $(F=0.2$, $\mathrm{df}=1 / 12, P=0.7$ ).

There were significant correlations between the MADRS score both at baseline and during IFN $\alpha$ based therapy and baseline serum PEP $(r=-0.40$, $\left.R^{2}=15.9 \%, \quad F=9.6, \quad \mathrm{df}=1 / 51, \quad P=0.003\right)$ and DPP IV $\left(r=-0.39, R^{2}=14.9 \%, F=9.5\right.$, $\mathrm{df}=1 / 51, P=0.004)$. There were significant correlations between the HAM-A scores both at baseline and during treatment and either baseline serum PEP $\left(r=-0.40, R^{2}=15.8 \%, F=9.6\right.$, $\mathrm{df}=1 / 51, P=0.003)$ and DPP IV $(r=-0.40$, $\left.R^{2}=15.7 \%, \quad F=9.5, \quad \mathrm{df}=1 / 51, \quad P=0.003\right)$. There was also a significant and positive correlation between serum PEP and DPP IV values at baseline and during treatment $\left(r=-0.45, R^{2}\right.$ $=20.6 \%, F=13.3, \mathrm{df}=1 / 51, P=0.0009$ ).

We have also examined the differences in baseline serum PEP and DPP IV between the norma controls and the hepatitis C patients. ANOVA showed that there were no significant differences in serum PEP between normal controls (mean $=0.299 \pm 0.069 \mathrm{U} / \mathrm{ml}$ ) and hepatitis $C$ patients (mean $=0.254 \pm 0.068 \mathrm{U} / \mathrm{ml})(F=2.7$, $\mathrm{df}=1 / 23, P=0.1$ ); and serum DPP IV between normal controls $($ mean $=45.6 \pm 12.0 \mathrm{U} / \mathrm{ml})$ and hepatitis $C$ patients $($ mean $=42.9 \pm 9.5 \mathrm{U} / \mathrm{ml}$ ) $(F=0.4, \mathrm{df}=1 / 25, P=0.5)$. However, patients with hepatitis-C with serum PEP $\leq 0.240 \mathrm{U} / \mathrm{ml}$ and DPP IV $\leq 38.9 \mathrm{U} / \mathrm{ml}$ activities had significantly lower levels of PEP and DPP IV, respectively, than normal controls.

\section{Discussion}

The main findings of the present study are that lowered baseline serum PEP and DPP IV are related to greater depressive and anxiety ratings during IFN $\alpha$ treatment and that lower baseline serum DPP IV is related to greater increases is IFN $\alpha$-induced depressive symptoms. These findings extent those of previous reports that major depression is accompanied by lowered serum DPP IV and PEP activity (1-3); and that a lowered baseline serum PEP activity in women at the end of term predicts the occurrence of postpartum depression (11).

We have hypothesized that lowered DPP IV and/or PEP activity may be related to the pathophysiology of depression. Thus, a lowered degradation and, consequently, increased levels of behaviorally active neuropeptides, such as substance P, neuropeptide Y, AVP, TRH, oxytocin, bradykinin and neurotensin, may be related to depression and anxiety (see Introduction). Also the lowered degradation of some pro-inflammatory cytokines, such as IL-1 $\beta$ and IL-6, together with alterations in lymphocyte activation and the production of cytokines, such as IL-1 $\beta$ and IL-2, could play a role in the link between peptidases and depression (see Introduction).

The following findings suggest that lowered baseline serum PEP and DPP IV may be relevant to the pathophysiology of depression. In depression, there is a significant inverse correlation between serum PEP activity and postdexamethasone adrenocorticotropic hormone (ACTH) and cortisol values, suggesting that lower serum PEP activity may play a role in the hyperactivity of the hypothalamic-pituitary-adrenal (HPA)-axis. One explanation is that a decreased degradation of AVP may stimulate the HPA-axis (3). Significant PEP activity is observed in the hypophysial-portal plasma, the median eminence and the central cortex (21). In sheep, the brain appears to be a major source of plasma PEP (22). Smaller neuropeptides, such as AVP, oxytocin and neurotensin, can penetrate the brain to exert their central activity, and, consequently, their peripheral degradation by PEP may be relevant to brain function 


\section{Maes and Bonaccorso}

(22). In major depression, significant correlations were observed between lowered serum DPP IV activity and signs of inflammation, such as lowered serum albumin and zinc (23).

There is now some evidence that IFN $\alpha$-induced activation of the cytokine network and changes in the serotonergic system can play a role in the development of IFNa-induced depressive symptoms (24). Thus, IFN $\alpha$-based immunotherapy significantly activates monocytes and $\mathrm{T}$-helper cells to produce more pro-inflammatory cytokines, such as IL-6 and IFN $\gamma$, which are depressogenic and anxiogenic $(10,24)$. IFNa-based immunotherapy significantly reduces plasma tryptophan (25) and serum 5-HT concentrations and increases serum kynurenine (25). Increases in serum kynurenine following IFN $\alpha$-immunotherapy give an estimation of the activity of indoleamine 2,3 dioxygenase (IDO), the enzyme which induces the catabolism of tryptophan into kynurenine. Indeed, IDO is directly induced by IFN $\gamma$, while IFN $\alpha$ has a weak direct effect and more important indirect effects through IFN $\alpha$-induced increases in IFN $\gamma$ and in a $15 \mathrm{kD}$ protein, which induces IDO $(26,27)$. Most importantly, significant relationships were observed between the depression and anxiety ratings following IFN $\alpha$ and the activation of the cytokine network (positive), serum kynurenine (positive) and serum 5-HT (negative). These findings suggest that the etiology of immunotherapy-induced depression is, in part, related to: increases in pro-inflammatory cytokines, such as IL-6 and IFN $\gamma$, which are depressogenic; lowered availability of tryptophan (28); increases in kynurenine, which is a neuroactive substance with anxiogenic and depressogenic properties (29); and other effects of IFN $\alpha$ on the serotonergic system such as increases in 5-HT transporter mRNA and the uptake activity of the 5-HT transporter (30). Moreover, the results of the present study suggest that lowered baseline serum PEP and DPP IV are predisposing factors to develop depression and anxiety following IFN $\alpha$ immunotherapy, probably through a reduced processing of behaviorally active peptides and cytokines. Of course, some other mechanisms may be involved, such as the effects of IFN $\alpha$ inducing thyroid autoimmunity and dysfunction (31). Moreover, such effects could be aggravated by the lowered acivity of TRH degrading peptidases, such as PEP.

\section{Acknowledgments}

This work was supported by a grant from the National Council for Research n.203.04.17 of 09/06/97. We would like to thank S. Scharpe, I. DeMeester, C. Durinx, F. Goossens, V. Marino,
A. Puzella, M. Pasquini, M. Biondi, M. Artini, and C. Almerighi for their valuable help.

\section{References}

1. Maes M, De Meester I, Vanhoof $G$ et al. Decreased serum dipeptidyl peptidase IV activity in major depression. Bio Psychiatry 1991;30:577-586.

2. Elgun S, Keskinege A, Kumbasar H. Dipeptidyl peptidase IV and adenosine deaminase activity. Decrease in depression. Psychoneuroendocrinol 1999;24:823-832.

3. Maes M, Goossens F, Scharpe S, Meltzer Hy, D'Hondt P, Cosyns P. Lower serum prolyl endopeptidase enzyme activity in major depression: further evidence that peptidases play a role in the pathophysiology of depression. Biol Psychiatry 1994;35:545-552.

4. Goossens F, De Meester I, Vanhoof G, Scharpe S. Distribution of prolyl oligopeptidase in human peripheral tissues and body fluids. Eur J Clin Chem Clin Biochem 1996;34:17-22.

5. De MeEster I, DurinX C, Bal G et al. Natural substrates of dipeptidyl peptidase IV. In: Langner J, ANsorge S, eds. Cellular peptidases in immune functions and diseases 2 New York: Plenum Publishers, 2000:67-87.

6. Van Hoof G, Goossens F, De Meester I, Hendriks D, Scharpé $S$. Proline motifs in peptides and their biological processing. FASEB J 1995:9:736-744.

7. De Meester I, Korom S, Van Damme J, Scharpe S. CD26, let it cut or cut it down. Immunol Today 1999;20:367-375.

8. Schon E, Demuth H-U, Erchmann E et al. Dipeptidyl peptidase IV in human $T$ lymphocytes. Impaired induction of interleukin 2 and gamma interferon due to specific inhibition of dipeptidyl peptidase IV. Scand J Immunol 1989; 29:127-132.

9. TANAKA T, DuKE-Cohan JS, Kameoka J et al. Enhancement of antigen-induced T-cell proliferation by soluble CD26/dipeptidyl peptidase IV. Proc Natl Acad Sci U S A 1994;91: 3082-3086.

10. Maes $M, C_{A}$ aruron $L, R_{A V A u d} A$ et al. Immunochemotherapy with interleukin-2 and/or interferon-alpha in cance patients induces lowered serum dipeptidyl peptidase IV activity, which is related to therapy-induced depressive symptoms and immune activation. Neuropsychopharmacology 2001;24:130-140.

11. Maes M, LibBrecht I, LiN A et al. Effects of pregnancy and delivery on serum prolyl endopeptidase (PEP) activity: higher serum PEP is related to anxiety in the early puerperium and a past history of depression, and lower serum PEP to postpartum depression. J Affect Disord 2000;57: 125-137.

12. MAES M. Major depression and activation of the inflammatory response system. Adv Exp Med Biol 1999;461:2546.

13. Maes M, Song C, Lin AH et al. Negative immunoregulatory effects of antidepressants: inhibition of interferon-gamma and stimulation of interleukin-10 secretion. Neuropsychopharmacology 1999;20:370-379.

14. Kubera M, Lin A, Bosmans E, Kenis G, Maes M. Negative immunoregulatory effects of antidepressants. J Clin Psychopharmacol 2001:21:199-206.

15. Bonaccorso S, Meltzer HY, Maes M. Psychological and behavioral effects of interferons. Curr Opin Psychiatry 2000;13:637-677.

16. Maes M, Bonaccorso S, Marino $V$ et al. Treatment with interferon-alpha (IFNalpha) of hepatitis $\mathrm{C}$ patients induces lower serum dipeptidyl peptidase IV activity, which is related to IFNalpha-induced depressive and anxiety 
symptoms and immune activation. Mol Psychiatry 2001;6:475-480.

17. Montgomery SA, Asberg A. A new depression scale designed to be sensitive to change. Br J Psychiatry 1979;134:382-389.

18. Hammton $\mathrm{M}$. The assessment of anxiety by rating. $\mathrm{Br} \mathrm{J}$ Psychiatry 1959;32:50-55.

19. Goossens F, De Meester I, Vanhoof G, Scharpé S. A sensitive method for the assay of serum prolyl endopeptidase. Eur Clin Chem Clin Biochem 1992;30:235-238.

20. Nagatsu T, Hino M, Fuyamada $H$ et al. New chromogenic substrates for X-Pro dipeptidyl aminopeptidase. Anal Biochem 1976;74:466-476.

21. Kato T, Orada M, Nagatsu T. Distribution of post-proline cleaving enzyme in human brain and the peripheral tissues. Mol Cell Biochem 1980;32:117-121.

22. Lawrence AC, Clark IJ, Campbell. DJ. Increased angiotensin-(1-7) in hypophysial-portal plasma of conscious sheep Neuroendocrinology 1992;55:105-114.

23. Maes M, DeMeester I, Verkerk R et al. Dipeptidyl peptidase serum activity in treatment resistant depression: relationships with immune-inflammatory markers. Psychoneuroships with immune-inflammator

24. Bonaccorso S, PuZzlla A, Marino V et al. Immunotherapy with interferon-alpha in patients affected by chronic hepatitis $\mathrm{C}$ induces an intercorrelated stimulation of the cytokine network and an increase in depressive and anxiety symptoms. Psychiatry Res 2001;105:45-55.
25. Bonaccorso S, Marino V, Puzella A et al. The increased depressive ratings in hepatitis-C patients receiving interferon-alpha (IFN $\alpha$ )-based immunotherapy are related to IFN $\alpha$-induced changes in the serotonergic system. J Clin Psychopharmacol 2002;22:86-90.

26. TAYLOR MW, FENG G. Relationship between interferongamma, indolamine 2,3-dioxygenase, and tryptophan catabolism. FASEB J 1991;5:2516-22.

27. ReCHT M, BoRden EC, KNIGHT E. A human 15-kDa IFN induced protein induces the secretion of IFN-gamma. J Immunol 1991;147:2617-23.

28. MAes M, MELTZER HYM. The serotonin hypothesis of major depression. In: Bloom FE, KuPFer DJ, eds. Psychopharmacology the fourth generation of progress. New York: Raven Press, 1995:933-944.

29. VeCSEI L, BEAL MF. Influence of kynurenine treatment on open-field activity, elevated plus-maze, avoidance behaviors and seizures in rats. Pharmacol Biochem Behav 1990 37:71-76.

30. Morikawa O, Sakai N, Obara $\mathrm{H}$, Sarto N. Effects of interferon-alpha, interferon-gamma and cAMP on the transcriptional regulation of the serotonin transporter. Eur $J$ Pharmacol 1998;349:317-324.

31. Murdolo G, Francisci D, Forin $F$ et al. Expression of endocrine autoantibodies in chronic hepatitis $C$, before and after interferon-alpha therapy. J Endocrinol Invest 2002;25:938-946. 


\title{
Chapter 9
}

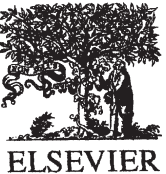

Research report

\section{Depression induced by treatment with interferon-alpha in patients affected by hepatitis $\mathrm{C}$ virus}

\author{
Stefania Bonaccorso ${ }^{\mathrm{a}, \mathrm{d}}$, Valentina Marino ${ }^{\mathrm{a}}$, Massimo Biondi ${ }^{\mathrm{a}}$, Franca Grimaldi ${ }^{\mathrm{b}}$, \\ Flora Ippoliti ${ }^{\mathrm{c}}$, Michael Maes ${ }^{\mathrm{d}, \mathrm{e}, \mathrm{f}, *}$ \\ 'Psychiatric Hospital, University 'La Sapienza', Rome, Italy \\ ${ }^{b}$ Department of Infectious and Tropical Diseases, University 'La Sapienza', Rome, Italy \\ 'Department of Immunology, University 'La Sapienza', Rome, Italy \\ ${ }^{\mathrm{d} C l i n i c a l}$ Research Center for Mental Health (CRC-MH), Antwerp, Belgium \\ ${ }^{e}$ Department of Psychiatry and Neuropsychology, University Hospital of Maastricht, Postbus 5800, 6202 AZ Maastricht, \\ The Netherlands \\ 'Department of Psychiatry, Vanderbilt University, Nashville, TN, USA
}

\begin{abstract}
Background: Several studies found a high incidence rate of neuro-psychiatric complications during long-term therapy with interferon alpha $(\operatorname{IFN} \alpha)$, e.g. slowness, severe fatigue, hypersomnia, lethargy, depressed mood, mnemonic troubles, irritability, short temper, emotional lability, social withdrawal, and lack of concentration. The aim of this study was to examine the incidence of depressed mood and major depression in patients who were treated with IFN $\alpha$. Methods: 30 patients, affected by chronic active $\mathrm{C}$-hepatitis, have been evaluated at baseline and 3 months after IFN $\alpha$ treatment. The evaluation consisted of psychometric assessments employing the DSM-IV criteria and the Montgomery Asberg Depression Rating Scale (MADRS). Results: At end-point, $40.7 \%$ of the patients suffered from a full blown major depression, according to the DSM-IV criteria for major depression. IFN $\alpha$ treatment induced a significant increase in the MADRS score from baseline to 3 months later. The MADRS items which were significantly increased at end-point were: expressed and unexpressed sadness; irritability; insomnia; loss of appetite; and asthenia. Discussion: The results show that prolonged IFN $\alpha$ treatment may induce depressive symptoms and major depression in a considerable number of subjects.
\end{abstract}

(c) 2002 Published by Elsevier Science B.V.

Keywords: Inteferon-alpha; Depression; Cytokines; Immunology; Cytokine treatment

\section{Introduction}

${ }^{*}$ Corresponding author. Tel.: + 31-43-387-5444; fax: + 31-43387-7443.

E-mail address: crc-mharonline.be (M. Maes).
Several studies report that repeated administration of interferon alpha (IFN $\alpha$ ) to patients with chronic active hepatitis may induce adverse effects, such as a flu-like syndrome with myalgias, fever, chills, arth-

0165-0327/02/\$ - see front matter (C) 2002 Published by Elsevier Science B.V.

PII: S0165-0327(02)00264-1 
ralgias and headaches (Vial and Descotes, 1994; Dusheiko, 1997). These symptoms commonly occur within a few hours following administration. Other authors (Renault et al., 1987; Adams et al., 1984; Hardy, 1996) found a high incidence rate (around $30 \%$ ) of neuro-psychiatric complications during long-term IFN $\alpha$ therapy, e.g. slowness, severe fatigue, hypersomnia, lethargy, depressed mood, mnemonic troubles, irritability, short temper, emotional lability, social withdrawal, and lack of concentration. These effects are dose- (between 3 and $10 \mathrm{MU}$ ) and timedependent and appear usually between the first and the third month after starting therapy. Janssen et al. (1994) reported three attempted suicides, one successful, during, or shortly after, IFN $\alpha$ therapy for chronic viral hepatitis. An Italian survey (Fattovich et al., 1996) performed on 11241 patients with chronic viral hepatitis (either B or C hepatitis) showed that life-threatening psychiatric side-effects occurred in a minute percentage of the patients treated. In that study, two persons, both with a history of depressive feelings, attempted to commit suicide 4-6 weeks after starting IFN $\alpha$ therapy. Rifflet et al. (1996) found that of 219 hepatitis patients treated with IFN $2 \%$ attempted suicide.

However, few studies have monitored the psychiatric complications after prolonged IFN $\alpha$ administration using standardized rating scales. Most studies evaluated neuropsychological functions, e.g. memory functions and visuo-spatial and visuo-construction ability. Some of these studies reported that IFN $\alpha$ treatment caused cognitive deterioration (Pavol et al., 1995; Mattson et al., 1984). One study (McDonald et al., 1987) assessed psychiatric morbidity with a selfadministered questionnaire to detect psychiatric morbidity and psycho-social functioning. IFN $\alpha$ increased psychiatric morbidity: the psychiatric symptoms seem to be an accentuation of existing symptoms such as anxiety, depression, fatigue, and impaired concentration.

There is now evidence that major depression is accompanied by activation of the inflammatory response system (IRS) with an increased production of proinflammatory cytokines, such as interleukin-1 (IL-1), IL-6 and IFN $\gamma$ (review: Maes, 1995, 1999). In experimental animals, increased production of proinflammatory cytokines or administration of these cytokines may induce depression-like symptoms and depression (Maes, 1999; Maier and Watkins, 1998; Connor and Leonard, 1998). Since IFN $\alpha$ induces IRS activation (Cavaillon, 1996), it may be hypothesized that IFN $\alpha$ administration could induce depression (Maes, 1995; Yirmiya, 1997).

The aims of the present study were to examine the incidence of depressive symptoms and major depression in patients treated with IFN $\alpha$ for chronic hepatitis $C$.

\section{Subjects and materials}

Thirty consecutive patients (24 males and six women; mean ( \pm S.D.) age $=56.2 \pm 10.1$ years) affected by chronic active $C$-hepatitis have been examined at the Department of Infectious and Tropical Diseases at the University of Rome 'La Sapienza' between November 97 and May 98. Patients with coexisting viral hepatic disease such as hepatitis $B$ and D, Epstein Barr, and cytomegalovirus, autoimmune diseases, and patients treated with immunosuppressive therapy, patients with psychoactive substance use disorders, and patients with a past or present history of axis-I disorders have been excluded. Illness onset and awareness ranged from a minimum of two to a maximum of 5 years before inclusion in the present study. The study design was approved by the local ethical committee and the subjects gave written informed consent after the study protocol was fully explained.

Treatment consisted of IFN $\alpha, 3 \mathrm{MU}$, administered three times a week. The subjects have been evaluated before therapy (baseline) and after 3 months of treatment (end-point). The evaluation consisted of psychometric assessments by means of the Montgomery Asberg Depression Rating Scale (MADRS), a 10-item semi-structured standard mental state examination. The diagnosis of major depression was made by two psychiatrists, according to the DSM-IV criteria for a major depressive episode. Differences between baseline and end-point MADRS scores were assessed by means of repeated measure design analyses of variance (ANOVA). There were no changes either in the physical status or in the pharmacological treatment of the patients between baseline and end-point. 


\section{Results}

Three patients were excluded: one due to text uncomprehension, and two because they were treated with thyroxine or insulin. According to DSM-IV criteria not one of the patients suffered from major depression at baseline. Even the subjects with increased MADRS scores (Fig. 1) at baseline did not fulfil the diagnostic criteria of major depression a that time. These higher scores at baseline may be explained by demoralization or adjustment disorde due to the awareness of the physical illness by the patients. Up to $40.7 \%$ of the patients suffered from a full blown major depression at end-point. At endpoint, these patients reached a final MADRS score of $12-28$ points.

The total MADRS score and the MADRS items are significantly higher $(F=15.5, \mathrm{df}=1 / 26, P=$ $0.0008)$ at end-point (mean $=11.78 \pm 9.28)$ than at baseline (mean $=5.81 \pm 9.28)$. Table 1 shows the effects of IFN $\alpha$ on the MADRS items. Expressed and unexpressed sadness, irritability, insomnia, loss of appetite and asthenia are significantly higher at end-point than at baseline. A trend towards a signifi-

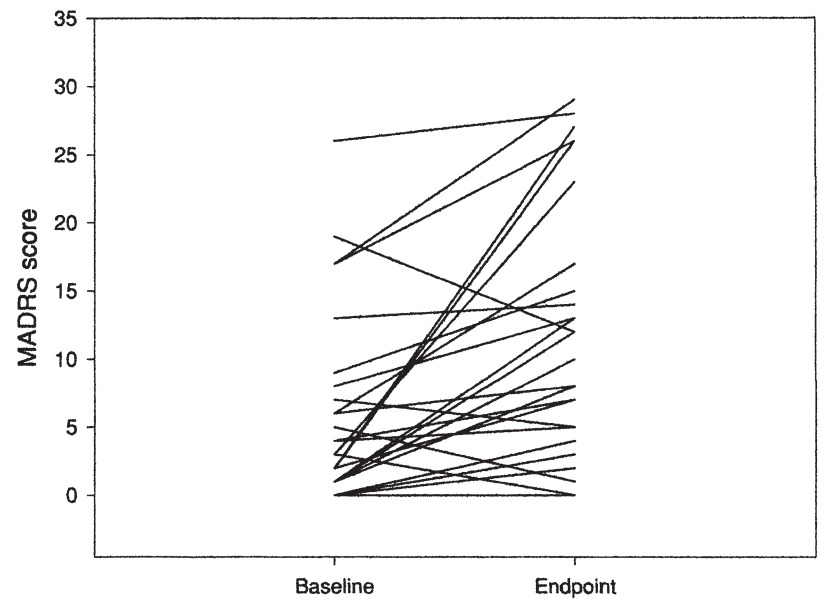

Fig. 1. Measurements of the total Montgomery Asberg Depression Rating Scale (MADRS) score in 27 patients both before (baseline) and 3 months after treatment (end-point) with interferon- $\alpha$.

Table 1

Measurements of the MADRS item scores both before (baseline) and after treatment with interferon-alpha during 3 months (end-point)

\begin{tabular}{lllll}
\hline Items & Baseline & End-point & $F$-value & $P$-value \\
\hline 1. Expressed sadness & $0.93(1.17)$ & $1.70(1.38)$ & 7.5 & 0.01 \\
2. Unexpressed sadness & $0.96(1.25)$ & $1.81(1.61)$ & 7.8 & 0.009 \\
3. Irritability & $0.88(0.97)$ & $1.81(1.52)$ & 10.0 & 0.004 \\
4. Insomnia & $0.59(0.84)$ & $1.33(1.3)$ & 5.4 & 0.03 \\
5. Loss of appetite & $0.15(0.53)$ & $0.62(1.11)$ & 6.6 & 0.02 \\
6. Lack of concentration & $0.62(1.24)$ & $1.22(1.69)$ & 3.2 & 0.08 \\
7. Asthenia & $0.40(0.97)$ & $1.74(1.43)$ & 22.3 & 0.0002 \\
8. Anhedonia & $0.48(0.84)$ & $0.88(1.67)$ & 2.0 & 0.2 \\
9. Pessimistic thoughts & $0.55(0.84)$ & $0.55(1.05)$ & 0.0 & 0.99 \\
10. Suicidal thoughts & $0.22(0.80)$ & $0.07(0.26)$ & 6.8 & 0.6 \\
\hline
\end{tabular}

All results are shown as mean ( \pm S.D.). All results of repeated measure design ANOVAs $(\mathrm{df}=1 / 26)$. 
cant increase was observed for lack of concentration. There were no significant gender-related differences in the effects of IFN $\alpha$ on the MADRS items. We used no $P$-corrections to adjust for multiple comparisons. However, the primary analyses showed an increase in the total MADRS score, while the MADRS items are highly intercorrelated. This allows further analyses at the item level without $P$ correction.

\section{Discussion}

Our results show that $40.7 \%$ of the hepatitis patients became clinically depressed, according to DSM-IV criteria, after 3 months treatment with IFN $\alpha$. Moreover, the total MADRS score and the MADRS items irritability, sadness, insomnia, loss of appetite and asthenia were significantly increased by treatment with IFN $\alpha$. One limitation of the study design is the absence of a control group. This could have hampered the interpretation of the results that the increased incidence of major depression is attributable to the effects of IFN $\alpha$ treatment rather than to some aspects of disease process or of adjustment. However, due to ethical considerations, a placebocontrolled study cannot be carried out. Moreover, no changes either in the physical status or in the use of other drugs were recorded during the 3-month treatment period. In addition, illness onset and awareness ranged from a minimum of two to a maximum of 5 years before inclusion in the present study.

These findings suggest that the increase in depressive symptoms and the development of major depression are related to IFN $\alpha$ treatment rather than to demoralization or deterioration in physical status. Since IFN $\alpha$ administration may induce IRS activation, the results of the present study are in agreement with the hypothesis that activation of the IRS may induce depression (Maes et al., 1995; Yirmiya, 1997).

The exact mechanism whereby IFN $\alpha$ may induce depression has remained elusive. IFNs (alpha and gamma) can enter the CNS from the periphery, i.e. the cervical, thoracic and lumbosacral segments of the spinal cord and the brain (Pan et al., 1997). When IFN $\alpha$ is injected systemically it can pass the bloodbrain barrier and reach effective concentrations in the brain (Dafny et al., 1988). Endothelial transcytosis is a possible mechanism whereby IFNs can enter the brain. Yamada and Yamanaka (1995) found IFN $\alpha$ receptors in microglial cells as well as in macrophages in infarct areas. Experiments in vitro showed that astrocytes can produce IFN $\alpha$ when treated with Newcastle Disease Virus (Lieberman et al., 1989). Central as well as peripheral administration of IFN significantly increases the firing rate of the somatosensory cortex, hippocampus, amygdala and the parafascicular nucleus and the majority of the ventromedial-hypothalamic nuclei (Dafny et al., 1996). Other studies suggest that IFN $\alpha$ modulates neuroendocrine functions. For example, IFN $\alpha$ induces the release of corticotrophin releasing factor (CRF) and arginine vasopressin (AVP) from the hypothalamus (Raber et al., 1997). IFNs also modulate the expression of serotonin transporter mRNA in the brain (Morikawa et al., 1998). It is thought that IRS activation and administration of cytokines, such as interferons, may induce depression through their modulation of the serotonergic system and through their effects on the hypothalamic-pituitary-adrenal axis (Maes, 1999; Yirmiya, 1997).

\section{Acknowledgements}

This work was supported by a grant from the National Council for Research no. 203.04.17 of 09/ 06/97.

\section{References}

Adams, F., Quesada, J.R., Gutterman, J.U., 1984. Neuropsychiatric manifestations of human leukocyte interferon therapy in patients with cancer. J. Am. Med. Assoc. 252, 938-941.

Cavaillon, J.-M., 1996. Interleukin-6. Les Cytokines. Masson, Paris.

Connor, T.J., Leonard, B.E., 1998. Depression, stress and immunological activation: the role of cytokines in depressive disorders. Life Sci. 62, 583-606.

Dafny, N., Lee, J.R., Dougherty, P.M., 1988. Immune response products alter CNS activity: interferon modulates central opioid function. J. Neurosci. Res. 19, 130-139.

Dafny, N., Prieto-Gomez, B., Dong, W.-Q., Reyes-Vazquez, C., 1996. Interferon modulates neuronal activity recorded from the hypothalamus, thalamus, hippocampus, amygdala and the somatosensory cortex. Brain Res. 734, 269-274. 
Dusheiko, G., 1997. Side effects of alpha interferon on chronic hepatitis C. Hepatology 3,121.

Fattovich, G., Giustina, G., Favarato, S., Ruol, A., Investigators of the Italian Association for the Study of the Liver, 1996. A survey of adverse events in 11241 patients with chronic viral $\mathrm{B}$ or $\mathrm{C}$ hepatitis treated with alfa interferon. J. Hepatol. 38-47.

Hardy, P., 1996. Trouble depressifs et interferon alpha. Gastroenterol. Clin. Biol. 20, 255-257.

Janssen, H.L.A., Brouwer, J.T., van der Mast, R.C., Schalm, S.W., 1994. Suicide associated with alfa-interferon therapy for chronic viral hepatitis. J. Hepatol. 21, 241-243.

Lieberman, A., Pitha, P.M., Shin, H.S., Shin, M.L., 1989. Production of tumor necrosis factor and other cytokines by astrocytes stimulated with lipopolysaccharide or a neurotropic virus. Proc. Natl. Acad. Sci. USA 96, 6348-6352.

Maes, M., 1995. The interleukin hypothesis of major depression. Prog. Neuropsychopharmacol. Biol. Psychiatry 19, 11-38.

Maes, M., 1999. Major depression and activation of the inflammatory response system. Adv. Exp. Med. Biol. 461, 25-

Maes, M., Smith, R., Scharpe, S., 1995. The monocyte-T lymphocyte hypothesis of major depression. Psychoneuroendocrinology $20,111-116$.

Maier, S.F., Watkins, L.R., 1998. Cytokines for psychologists: implications of bidirectional immune-to-brain communication for understanding behavior, mood, and cognition. Psychol. Rev. 105, 83-107.

Mattson, K., Niiranen, A., Laaksonen, R., Cantell, K., 1984 Psychometric monitoring of interferon neurotoxicity. Lancet (8371), 275-276.

McDonald, E.M., Mann, A.H., Thomas, H.C., 1987. Interferons mediators of psychiatric morbidity. An investigation in a trial of recombinant alpha-interferon in hepatitis-B carriers. Lancet 21, 1175-1177.

Morikawa, O., Sakai, N., Obara, H., Saito, N., 1998. Effects of interferon-alpha, interferon-gamma and CAMP on the transcriptional regulation of the serotonin transporter. Eur. J. Pharmacol. 349, 317-324.

Pan, W., Banks, W.A., Kastin, A.J., 1997. Permeability of bloodbrain and blood-spinal cord barriers to interferons. J. Neuroimmunol. 76, 105-111.

Pavol, M.A., Meyers, C.A., Rexer, B.S. et al., 1995. Pattern of neurobehavioral deficits associated with interferon alfa therapy for leukemia. Neurology 45, 947-950.

Raber, J., Koob, G.F., Bloom, F.E., 1997. Interferon alpha and transforming growth factor-beta-1 regulate corticotropin-releasing factor release from the amygdala: comparison with the hypothalamic response. Neurochem. Int. 30, 455-463.

Renault, P.F., Hoofnagle, J.H., Yoon Park, R.N., Mullen, K.D. Peters, M., Jones, B., Rustgi, V., Jones, A., 1987. Psychiatric complications of long-term interferon alfa therapy. Arch. Intern. Med. 147, 1577-1580.

Rifflet, H., Vuillemin, R.H., Oberti, F., Laine, P., Cales, P., 1996 Interferon et suicide au cours des hepatites virales chroniques. Gastroenterol. Clin. Biol. 2, 68.

Vial, T., Descotes, J., 1994. Clinical toxicity of the interferons. Drug Saf. 10, 115-150.

Yamada, T., Yamanaka, I., 1995. Microglial localization of alphainterferon receptor in human brain tissues. Neurosci. Lett. 189, 73-76.

Yirmiya, R., 1997. Behavioral and psychological effects of immune activation: Implications for depression due to general medical condition. Curr. Opin. Psychiatry 10, 470-476. 
82 Cytokines and Depression: A Neurochemical Hypothesis 


\section{Discussion}

The immune system is triggered not only in major depressive disorder but also in other diseases where depression is present in comorbidity or as a clinical symptom, such as somatization disorder (Rief et al., 2001), fibromyalgia (Bonaccorso et al., 1998), or for example in a group of detoxified chronic alcoholic patients without apparent liver disease (Maes et al., 1998), or a subgroup of patients affected by sleep disorders (Song et al., 1998) and patients with Alzheimer Disease (Bonaccorso et al., 1998).

In the somatization disorder (Rief et al., 2001), the concentrations of CD8 (a molecule secreted by activated T-lymphocytes) and IL- 6 (a pro-inflammatory cytokine) were significantly lower than the ones obtained in the group affected by major depression or healthy controls, while the receptor for IL-6 (IL-6R) had lower concentrations in the depression group than in the somatization group. The natural immunosuppresor and anti-inflammatory secretory protein Clara cell $16 \mathrm{KD}$ protein (CC16) was found to be significantly increased in patients affected by somatization syndrome than controls or patients affected by major depression. The receptor antagonist for IL-1 (IL-RA) was significantly elevated in all patients groups compared with controls. In this study immunological states of somatizing patients are significantly different from those of depressive patients, so in conclusion even if an immune activation is present this is consistently different from the one described for major depression. It's necessary to highlight this specificity in order to achieve a complete analysis of psychiatric clinical manifestations and the biological markers.

In fibromyalgia (Bonaccorso et al., 1998), even though there are not signs of immune activation as assessed by means of neopterin excretion in urine and, by inference, the comorbidity between both disorders and the presence of depressive symptoms in fibromyalgia patients may not be related to activation of cell-mediated immunity; however, lower concentrations of plasma tryptophan were observed. Moreover, a significant inverse correlation between tryptophan concentrations and morning pain responsiveness in those patients may suggest a direct involvement of lower tryptophan availability in the symptomatology of the disease. It is well known that cytokines, such as IFN-gamma, IL-1 and IL-2 may induce indoleamine 2, 3dioxygenase (IDO), a key enzyme which catabolizes tryptophan. Immune activation is accompanied by a decreased availability of tryptophan to the brain due to IDO-induced catabolism of tryptophan. Thus, the findings that there are no significant alterations in neopterin excretion, an index of IFNgamma-induced macrophage activity, do not exclude the existence of immune activation related to other pro-inflammatory cytokines, like IL-1 and IL-2.

In the group of detoxified alcoholic patients apparently without liver disease (AWLD) (Maes et al., 1998) significantly increased serum IL-8 concentrations and a trend toward higher IL-6 concentrations were found. IL-8 is produced by IL-1 and TNF-alpha induced monocytes, macrophages, neutrophils, endothelial cells, fibroblasts, hepatocytes and exerts a chemotaxic activity not only on neutrophils but also on eosinophils, basophiles, natural killer cells and lymphocytes. Thus increased serum IL-8 in detoxified AWLD is another indicant of activation of the IRS. Another important finding is that serum IL-1RA is significantly higher in detoxified AWLD patients than in non-alcoholic control subjects. IL-1RA is mainly derived from monocytes and is secreted, following activation, along with IL-1. Thus increased IL-8, IL-6 and IL-1 RA 
levels support the thesis that detoxification in AWLD patients is accompanied by an activation of the monocytic arm of cell-mediated immunity. Moreover, the lowered serum concentration of $\mathrm{CC}_{16}$, a molecule with immunosuppressive, anti-inflammatory, and anti-cytokine activities with inhibitory effects on phospholipase A2 activity and on the IL-2 related production of IFN-gamma, in detoxified alcoholic AWLD patients are supporting evidences of an activation of the IRS.

In the subgroups of patients affected by primary sleep disorders (Song et al., 1998) increased serum concentration of IL-6, IL-8, IL-1RA and an increased stimulated production of PGE2 were found. Alterations in sleep pattern like sleep deprivation or excessive daytime sleepiness are related to an increased IL-1, IL-2, IL-6, TNF-alpha as well as PGE2 concentrations (Norman et al., 1992; Vgontzal et al., Dinarello, 1987). The interaction between cytokines and sleep seems to be reciprocal. It has also been proposed by Pollmacher et al. (2002) a model according to which normal circulating levels of cytokines at physiological concentrations (in the order of the nanomolar concentrations) can promote physiological human functions such as the sleep-wake cycle while increased levels of circulating cytokines like TNF-alpha may induce a progressive decline in REM sleep amount.

Signs of activation of IRS such as increased concentrations of IL- 6 were also found in patients affected by Alzheimer's disease (Bonaccorso et al., 1998). This finding commensurates with earlier studies which described the implication of the inflammatory response system in the pathogenesis of DAT (Aisen et al., 1994; McGeer et al., 1995). In this respect, it has been hypothesized that DAT may result from an autoimmune process, characterized by either local or systemic increases in IL-6 production, which induces alphaz-macroglobulin to alter the processing of amiloid precursor protein and, consequently, the disposition of amiloid beta protein within the senile plaques.

Except for DAT and AWLD patients, in all the other groups studied, lowered values of plasma tryptophan and tryptophan/CAA ratio were found, along with IRS activation. For the patients affected by primary sleep disturbance these results were also accompanied by an inverse relationship between the availability of tryptophan to the brain and signs of the inflammatory system response activation, such as increased serum IL-6 and IL-1 RA indicating an enhanced catabolism cytokine-induced (IL-2 or IFN-gamma, IL-1) of tryptophan. Therefore the lowered availability of tryptophan might intervene with the serotonin neurotransmission and participate to the onset of mood disturbances.

In detoxified AWLD patients the negative results might be explained by the major upheavals in IRS function in AWLD that could have blurred any relationship between the IRS and plasma tryptophan. For DAT patients the results that show no significant alterations in the availability of tryptophan to the brain might suggest that the well documented serotonergic abnormalities in brain of DAT patients are probably not related to changes in the availability of tryptophan but on others serotonergic functions.

The results mentioned above show how the immune activation reflects a non-specific association to mood disturbances. However even if some of the variables found, such as IL-1RA, (Rief et al., 2001) showed positive correlations with other disorders where the depressive 
symptomatology is highly present in comorbidity such as somatization disorder, fibromyalgia, AWLD patients, DAT patients anxiety and sleep disorders, the presence of an immune activation cannot be taken as an unequivocal sign for depression. The biological changes involved in the pathophysiology of mood disturbances reach much higher complexity where a determinant role seems to be played by the serotonin neurotransmission and the alteration of the endocrine hypothalamic-pituitary-adrenal axis.

The updated information about this argument is summarized by a recent review (Anisman et al., 2003), which describes how severe depressive illness is frequently accompanied by signs of activation of the monocytic and T-lymphocitic arm of cell-mediated immunity; and by increased production of proinflammatory cytokines such as IL- 1 and IL- 6 which elicit sickness behavior in animals and symptoms of anxiety or depression in humans; and by changes in the neuroendocrine function and central neurotransmission induced by cytokines implicated in depression.

An interesting example of the complex interactions described above might be interferonalpha.

Interferon-alpha has been widely used for therapeutic purposes to treat patients affected by cancer or chronic viral diseases. Its administration alone or along with interleukin-2 has been reported strongly related to mood disturbances (Capuron et al., 2002). Depression or mood abnormalities induced by interferon-alpha immunotherapy normally occur after the first month of treatment while flu-like symptoms, fatigue, anorexia are normally detected earlier. Capuron (2002) searched for depressive symptoms after five days of IFN-alpha treatment with negative results. In fact, depression is not the only side effect related to IFN-alpha immunotherapy. In a wide survey on 642 patients affected by melanoma (Kirkwood et al., 2002), flu-like symptoms, elevated liver enzymes along with hypertrygliceridemia, fatigue, anorexia, myelosuppression, dermatological reactions were found to be symptoms correlated to IFN-alpha therapy in a dose-, time- and route of administration- depending manner. Fatigue was reported in $96 \%$ of patients. This finding is confirmed by another study (Malik et al., 2001) where fatigue was found to be the most common of the constitutional side effects, reported in the $70-100 \%$ of patients receiving IFN therapy and requiring a dose reduction in $10-40 \%$ when an interruption wasn't necessary. Since there is a consistent percentage of patients' interrupting the therapy because of the side effects, informative sessions and a therapeutic alliance between general practioner, psychiatrist and patients are required.

How interferon-alpha could interact with the central nervous system (CNS)?

Interferons $(\alpha$ and $\gamma$ ) can enter the CNS from the periphery in a regional selective manner (Pan et al., 1997). The permeability of the spinal cord is higher than that of the brain and for each region the permeability to IFN $\alpha$ is greater than that to IFN $\gamma$. The mechanism involved seems to be "endothelial transcytosis". Yamada et al. (1995) found IFN $\alpha$ receptors in microglia cells as well as in macrophages in cerebral infarct areas. Another system might be constituted by its production within the CNS; in fact, in vitro experiments showed that astrocytes produce IFN $\alpha$ when treated with Newcastle disease virus (Lieberman et al., 1989). In SpragueDawley rats IFN $\alpha$ modulated the majority of the hypothalamic (70\%), amygdala (76\%), hippocampus $(75 \%)$ and cortical $(82 \%)$ cells whether the route of administration was within 
the brain or given peripherally (i.v. or i.p.). IFN $\alpha$ in general suppressed the hypothalamic neuronal activity while accelerating neuronal activity in all the other CNS sites (Dafny et al., 1996; Raber et al., 1997).

In addition, IFN $\alpha$ may modulate physiological functions through opioid receptors (Menzies et al., 1992), and it seems to play an antagonistic role with naloxone in binding to membrane sites. IFN $\alpha$ 's interaction with opioid receptors could explain the attenuation of morphine withdrawal symptoms when IFN $\alpha$ is injected systemically one hour before naloxone (Dafny et al., 1988). Naloxone reduces the IFN $\alpha$ increased immobility time in the forced swimming test in the mouse (Makino et al., 2000). These evidences indicate that IFN $\alpha$ may modulate many functions in the central nervous system.

IFN-alpha treatment was also the object of the study we performed: 18 patients affected by chronic hepatitis $\mathrm{C}$ undergoing to an IFN-alpha treatment were evaluated before starting the treatment, 2 weeks after, 4 and 6 months after. The results showed (Bonaccorso et al., 2002) a significant increase in MADRS and HAM-A scores within 1 month after starting immunotherapy with IFN-alpha and that the depression and anxiety scores further increased during treatment up to 6 months where the protocol evaluation ended. These findings are in agreement with previous reports showing that IFN-alpha based immunotherapy increases depressive ratings and induces full-blown major depression in a considerable number of patients. Some authors argued that immunotherapy with IFN-alpha may aggravate the severity of preexisting symptomatology, such as anxiety, depression, fatigue and impaired concentration. Patients with a subclinical symptomatology may be more predisposed to IFN-alpha induced mood disturbances. However IFN-alpha induced major depression may also occur in a population with no subclinical symptoms and without a history of psychiatric disorders (Capuron et al., 1999; Rosenstein et al., 1999).

IFN-alpha seems to possess interesting interactions with the cytokine network as a therapeutic agent. In fact it has been found that patients affected by HCV virus who responded positively to the administration of the interferon-alpha (IFN-alpha) treatment (Naveau et al. 1999; Hayashi et al., 1995) show higher concentrations of sIL-2r than controls but lower than non-responders and also increased level of interleukin-4 (IL-4) compared to non-responders (Higashi et al., 1999). Even though the underlying mechanisms cannot be explained it seems that interferon-alpha treatment might elicit a Th1-type response. This immune response may help the organism in clearing the virus-infected hepatocytes, and once HCV is completely eliminated and Th1 cells not activated any longer, it may allow the Th2 system to reach higher levels and ameliorate the tissue-damaging effects of immune response. IFN-alpha is able to promote the cytokine network, in particular IL- 6 which was significantly and positively correlated to therapy-induced increases in MADRS and HAM-A scores. This result shows that the correlation between IL- 6 and clinical expression of depressive symptoms may give also positive confirmations to the role of IL- 6 as possible clinical indicator of future development of depressive symptomatology.

Although the hypothesis of a direct correlation between the IFN-alpha therapy the activation of the cytokine network and the depressive clinical symptomatology remains highly speculative, this represents a suggestive perspective. In our study we found that prolonged treatment with IFN-alpha increases also serum IL-10 (for a 6 months period evaluation) and 
serum IL-6 and IL-8 (for 2-4 weeks).

From our results it appears that IFN-alpha based immunotherapy induces the cytokine network as a whole rather than Th-1 vs. Th-2 cytokines (Bonaccorso et al., 2001). A possible explanation for the contradictory results is that the capacity of IL-10 production varies according to the genetic composition of the IL-10 locus while in most studies the IL-10 gene promoter polymorphism was not determined.

IFN-alpha directly or indirectly, may affect 5-HT metabolism and, thus, may induce depression. In fact plasma tryptophan (Bonaccorso et al., 2002) has been found to be significantly reduced and serum concentrations of kynurenine significantly increased during immunotherapy with IFN $\alpha$. The ratio between kynurenine and tryptophan also represents an indicator of serotonin metabolism. In our study, the K/T quotient was significantly increased during IFN $\alpha$ based immunotherapy. These findings suggest that IFN $\alpha$-based immunotherapy increases the catabolism of tryptophan to kynurenine. IFN $\alpha$, may induce indoleamine 2, 3 dioxygenase (IDO) (Taylor, 1991), the major tryptophan catabolizing enzyme in the brain, kidney, lung, spleen, duodenum and immune cells. While IFN $\gamma$ directly induces IDO, IFN $\alpha$ has a weak direct effect and an indirect effect through a $15 \mathrm{kD}$ protein, a product of IFN $\alpha$-treated monocytes and lymphocytes that stimulates IDO and IFN $\gamma$ production (Recht at al., 1991). Thus, activation of the IRS characterized by Th-1-like $T$ cell activation with hyperproduction of interferons induce IDO and, consequently, stimulates the catabolism of tryptophan, therefore decreases 5-HT availability and impairs the neurotransmission (Widner, 2000). It has been questioned whether plasma tryptophan might reflect the brain availability. Nevertheless, the influx of tryptophan into the brain depends on the plasma concentrations of all tryptophan, i.e. free and/or total, as well as on the concentrations of albumin (70-90\% of tryptophan is loosely bound to serum albumin) and the CAAs, as reflected by the tryptophan/CAA ratio (Curzon et al., 1975; Yuwiler, 1977).Thus, decreases in plasma tryptophan may reflect a decreased availability of plasma tryptophan to the brain. Interestingly, IFN $\alpha$-based immunotherapy has a significantly stronger effect on plasma tryptophan and on the K/T quotient in women than in men (Bonaccorso et al., 2002). These findings suggest that the catabolism of tryptophan into the kynurenine pathway is more sensitive to induction by pro-inflammatory cytokines in women than in men. These results are also in agreement with previous findings that the lowered availability of plasma tryptophan is more pronounced in depressed women than in depressed men (Maes \& Meltzer, 1995). These gender-related effects in the serotonergic system could play a role in the increased susceptibility of women to develop depression.

Noticeable is the fact that in our study also plasma levels of 5-HT were significantly diminished during IFN-alpha treatment. In animal and in vitro experiments, IFN $\alpha$ was found to have a suppressant effect on the 5-HT concentrations. In Wistar rats, decapitated $2 \mathrm{~h}$ after i.c.v. injection of IFN $\alpha$, the levels of $5-\mathrm{HT}$ were significantly reduced in the frontal cortex in a dosedependent manner, and the levels of both $5-\mathrm{HT}$ and 5 - $\mathrm{HIAA}$, the major 5 - $\mathrm{HT}$ catabolite, were reduced in the mid brain and the striatum (Kamata, 2000). In mice, IFN $\alpha$ for $3 \mathrm{~h}$ increase the levels of the 5-HT transporter mRNA in the midbrain and adrenal glands (Morikawa, 1998). Treatment with IFN $\alpha$ for $3 \mathrm{~h}$ increases the levels of 5 - $\mathrm{HT}$ transporter mRNA in human placental choriocarcinoma cells (BeWo cells) and this effect is inhibited by treatment with actinomycin D, an inhibitor of transcription (Morikawa, 1998). Exposure of primary carcinoid cell cultures with and without IFN $\alpha$ pretreatment to radiation (2 Gy and 8 Gy) showed that 
irradiation alone has no significant effect on the $5-\mathrm{HT}$ content of the medium and that when cells were preincubated with 1,000 IU/ml IFN $\alpha$ irradiation with $8 \mathrm{~Gy}$ induced a significant reduction of 5-HT concentrations in the medium on day 12 to 54.9 (Jacobsen, 1993). First, IFN $\alpha$ significantly increases both the $\mathrm{Kd}$ and Bmax measures of [3H]8-OH-DPAT binding at lowaffinity binding sites, but not at the high-affinity sites (Abe, 1999). These results suggest that IFN $\alpha$ affects the low-affinity 5-HT1A receptors sites. Acute $(1 \mathrm{~h})$ intraperitoneal and intracerebroventricular treatment with IFN $\alpha$ significantly inhibit wet-dog shakes induced by (+/-)-1-(2, 5-dimethoxy-4-iodophenyl)-2 aminopropane (DOI; $0.5,1.0 \mathrm{mg} / \mathrm{kg}$ ), which is mediated by $5-\mathrm{HT}_{2}$ receptor in rats (Kugaya, 1996). Thus, not only IFN $\alpha$-induced catabolism of tryptophan to kynurenine and diminished $5-\mathrm{HT}$ levels, but also changes in 5-HT1A receptor characteristics and $5-\mathrm{HT}_{2}$ receptor function may be involved in the development of IFN $\alpha$-induced depressive symptoms. Thus, the above results suggest that the IFN $\alpha$-induced depressive symptoms may arise through depletion of peripheral and central $5-\mathrm{HT}$, which in part may be related to changes in the $5-\mathrm{HT}$ transporter transcription and the uptake activity of the $5-\mathrm{HT}$ transporter or IDO activation and also might be related to changes in serotonin receptors activity and affinity.

IFN-alpha does not only affect the cytokine network and the 5-HT neurotransmission, but also the HPA axis. Interestingly, IFN $\alpha$ has been reported to induce the release of corticotropin releasing factor from neurons in the hypothalamus (Shuto, 1997). Increased CRH levels are found in patients with depression. The selective $\mathrm{CRH} 1$ receptor antagonist, $\mathrm{CP}-154526$, shows antidepressant like effect in rats with stress-induced depression-like behavior (Catalan et al., 1998; Mitchell, 1998; Mansbach et al., 1997). A study by Yamano and coworkers (2000) illustrated the activation of the $\mathrm{CRH} 1$ receptors and therefore of the HPA axis mediated by IFNalpha, suggesting an involvement of this cytokine in depression-like behavior in animals. The pretreatment with indomethacin and naloxone did not inhibit the activation of the $\mathrm{CRH}_{1}$ receptor indicating that prostaglandins and opioid system do not participate in this process. Moreover, a study conducted by Juengling (2000) and coworkers reported a hypometabolism in the frontal cortex in patients undergoing IFN-alpha treatment and these changes covaried with depression scores. The hypometabolism of the frontal cortex is an evidence normally found in depressed patients and in this case it might represent also a "vulnerability factor" for the development of depressive symptomatology of patients treated with IFN-alpha.

Summarizing, the IFN-alpha activities unveil: 1) impact on the cytokine network; 2) modulation of the serotonergic neurotransmission; 3) activation of the HPA axis. The above results suggest that IFN $\alpha$ should be considered a neuromodulator.

It has been questioned (de Beaurepaire, 2002) whether depression induced by cytokines is a typical depression. The essential features described by Freud were the feelings of worthlessness and guilt normally observed in melancholic patients and the persistence of these across time. We found that (Bonaccorso et al., 2002) prolonged IFN-alpha treatment may induce mood disturbances in $40.7 \%$ of the population examined with significantly increases sadness (expressed, unexpressed), irritability, insomnia, loss of appetite and asthenia. Even though the administration of a psychometric scale cannot entirely translate the clinical descriptions of depressive symptomatology, the results reported above indicate the absence of the classical core symptoms described by Freud. From this point of view, the depressive 
state occurring during cytokine therapy is more likely to belong to the DSM-IV category of "depression related to a general medical condition" (DSM-IV, 2001). Other findings (Owen et al., 2001) show that depression whether induced by infections ("organic" or "mood disorder due to a medical condition") or not (major depression, according to DSM-IV criteria, excludes all possible organic etiologies) is related to an increased production of cytokines (in particularly of IL-1beta and IL-6). Until now cytokines have been indicated as responsible at least for the vegetative symptoms of depression such as insomnia, weight loss, loss of appetite, psychomotor retardation, asthenia but further investigations are required to elucidate the association between cytokines and mood disorders.

We have to report the tremendous efficacy showed by antidepressive drugs in treating the depression occurred in patients treated with high dosage of cytokines such as IL-2 or IFNalpha. This finding is evidence in favor of the cytokine theory of mood disturbances cytokine induced.

Moreover, antidepressants drugs are widely known efficacious treatment for mood disturbances but recently they also have been indicated to possess antinflammatory activities. The main findings obtained in this regard show that clomipramine, sertraline and trazodone have a significant suppressive effect on IFN-gamma and a significant stimulatory effect on IL-10 secretion by whole blood stimulated with polyclonal activators (Maes et al., 1999). Since an increased production of pro-inflammatory cytokines such as IFN-gamma, alpha, IL-1beta, IL-2, IL- 6 and TNF-alpha may play a role in the etiology of depression while IL-10 potently suppresses the production of pro-inflammatory cytokines; the above findings explain the intricate links and connections in the cytokine network.

However, it might be questioned whether in vitro results might be translated into the in vivo conditions. It has been shown that subchronic treatment with fluoxetine, an SSRI, normalized the initially increased serum IL- 6 concentrations in depressed patients (Sluzewska et al., 1995) and in animals (Dredge et al., 1999; David et al., 2003). Moreover, Sammut et al. (2002) found in rats a reversible IFN-alpha induced anhedonia after the administration of desipramine and fluoxetine. Also, various case reports in humans have shown, in this respect, that the IFN $\alpha$-induced major depression may be well responsive to therapy with antidepressants, e.g. selective serotonin reuptake inhibitors and tricyclic antidepressants (Maddrey et al., 1999; Berk et al., 1999; Goldman et al., 1994).

The first mechanism by which antidepressive drugs may exert their activity is a normalization of the serotonergic neurotransmission. T lymphocytes express 5 - $\mathrm{HT}$ receptors such as 5$\mathrm{HT}_{1} \mathrm{~A}$ and $5-\mathrm{HT}_{2} \mathrm{~A} / 2 \mathrm{C}$ receptors as well as high affinity for $5-\mathrm{HT}$ transporter, whereas macrophages possess a specific active $5-\mathrm{HT}$ uptake system similar in activity to the observed for platelets. Moreover, 5 - $\mathrm{HT}$ has potent negative immunoregulatory effects such as a decrease in the mitogen-induced T lymphoproliferative response, suppression of DNA lymphocyte synthesis, inhibition of the migration of mononuclear lymphocytes, inhibition of $T$ cell activation of normal spleen and a decrease in the synthesis of TNF-alpha by macrophages. $5-\mathrm{HT} 1 \mathrm{~A}$ antagonists and inhibitors of $5-\mathrm{HT}$ synthesis may suppress IL-2 stimulated T cell proliferation and production of Th1-like cytokines including IFN-gamma.

In conclusion, the $5-\mathrm{HT}_{1} \mathrm{~A}$ and $5-\mathrm{HT}_{2} \mathrm{~A}$ receptors and their antagonists along with intracellular 5-HT depletion are able to suppress various aspects of immune action. Thus antidepressant drugs, such as tricyclics or SSRIs may be able to exert their antinflammatory activity through 
the serotonergic enhanced neurotransmission and therefore blockade of $5-\mathrm{HT} 2 \mathrm{a} / 2 \mathrm{c}$ receptors increased extracellular 5-HT and depletion of intracellular 5-HT stores (Kenis, 2002).

Even if not completely confirmed (for a review see Kenis and Maes, 2002), a second possible mechanism by which antidepressants might exert their activity on the immune system is the increase intracellular concentration of cyclic adenosine monophosphate (CAMP). It's generally believed that pharmacological augmentation of CAMP down regulates the expression of pro-inflammatory cytokines such as IFN-gamma and TNF-alpha and increases IL-10. Therefore cAMP levels induced by antidepressant treatments might exert in a decreased production of IFN-gamma and an enhanced secretion of IL-10.

In conclusion, accumulating evidence indicates that major depression is associated with an enhanced production of pro-inflammatory cytokines. Still it needs to be elucidated whether these cytokines may play a causative role. The role of antidepressant drugs in major depression manifested during cytokine therapy constitutes a positive clinical response to mood disorders for patients necessitating administration of high dosage of cytokines but still further researches are required to establish the exact correlation between these, the cytokine network and central neurotransmission.

Additional studies could be performed with the technique of the microdialysis to understand the neuromodulatory function of cytokines on monoamine dynamics, such as the dopaminergic and serotonergic and noradrenergic changes in the amygdala, dorsal raphe nucleus, and prefrontal cortex after chronic administration of IFN-alpha (or any other significant cytokine) to experimental animals in order to elucidated cerebral modifications not otherwise understandable.

Also, the clinical impact of antidepressants on subjects developing a depressive symptomatology during cytokine therapy needs to be clarified. Preliminary data on a group of patients affected by multiple sclerosis and major depression during treatment with IFN-beta showed a drastic reduction in HAM-D and HAM-A scores after a four months period of sertraline treatment (Bonaccorso, SOPSI 2003, Personal Communication). 


\title{
Cytokines en depressie: een neurochemische hypothese
}

\author{
Dr. Stefania Bonaccorso M.D.
}

\begin{abstract}
Er is steeds meer bewijs dat er een verband bestaat tussen het immuunsysteem en het centrale zenuwsysteem. Deze wederzijdse interactie duiden op een belangrijk gebied voor onderzoek dat kan leiden tot nieuwe inzichten in de etiologie van neuropsychiatrische aandoeningen. In dit proefschrift wordt de complexe relatie tussen het immuunsysteem, de endocriene functie, de hersenen en het gedrag van patiënten beschreven die aan hepatitis $C$ lijden en een depressieve symptomatologie vertonen, en daarvoor worden behandeld met interferon-alpha (IFN-alpha),. In dit proefschrift wordt een neurochemische hypothese gegeven die stelt dat niet alleen IFN-alpha effectief is als behandeling maar tegelijk ook een neuromodulator is.
\end{abstract}

Dit onderzoek werd financieel ondersteund door een beurs van de Italiaanse onderzoeksraad (National Council for Research n.302.04.17 of og/o6/97)

Trefwoorden: depressie, immuniteit, interferon-alpha

\section{Stefania Bonaccorso}

Address:

Viale Carlo Felice 63, 00185-Rome, Italy

Home Phone: +39-06-7009302

Cell Phone: +39-3335906200

Work Phone:+39-06-80345280

Fax number: +39-0774-401483 
92 Cytokines and Depression: A Neurochemical Hypothesis 


\section{References}

Abe S, Hori T, Suzuki T, Baba A, Shiraishi H, Yamamoto T. Effects of chronic administration of interferon alpha A/D on serotonergic receptors in rat brain. Neurochem Res. 1999 Mar;24(3):359-63.

Aisen PS, Davis KL. Inflammatory mechanisms in Alzheimer's disease: implications for therapy.Am J Psychiatry. 1994 Aug;151(8):1105-13.

Anisman H, Kokkinidis L, Borowski T, Merali Z. Differential effects of interleukin (IL)-1beta, IL2 and IL- 6 on responding for rewarding lateral hypothalamic stimulation. Brain Res. 1998 Jan 1;779(1-2):177-87.

Anisman H, Merali Z. Anhedonic and anxiogenic effects of cytokine exposure. Adv Exp Med Biol. 1999; 461:199-233.

Anisman H, Merali Z.Cytokines, stress and depressive illness: brain immune interactions. Ann Med. 2003; 35(1):2-11.

Arborelius L, Owens MJ, Plotsky PM, Nemeroff CB. The role of corticotropin-releasing factor in depression and anxiety disorders. J Endocrinol. 1999 Jan;160(1):1-12.

Berk L, van Gool AR, Bannink M, Kruit WH. Side effects of interferon alfa.Ned Tijdschr Geneeskd. 1999 Jul 10;143(28):1461-4.

Black PH. Immune system-central nervous system interactions: effect and immunomodulatory consequences of immune system mediators on the brain. Antimicrob Agents Chemother. 1994 Jan; 38(1):7-12.

Bluthe RM, Michaud B, Kelley KW, Dantzer R. Vagotomy attenuates behavioural effects of interleukin-1 injected peripherally but not centrally. Neuroreport. 1996 Jun 17; 7(9):1485-8.

Boje KM, Jaworowicz D Jr, Raybon JJ. Neuroinflammatory role of prostaglandins during experimental meningitis: evidence suggestive of an in vivo relationship between nitric oxide and prostaglandins. J Pharmacol Exp Ther. 2003 Jan; 304(1):319-25.

Bonaccorso S, Lin A, Song C, Verkerk R, Kenis G, Bosmans E, Scharpe S, Vandewoude M, Dossche A, Maes M. Serotonin-immune interactions in elderly volunteers and in patients with Alzheimer's disease (DAT): lower plasma tryptophan availability to the brain in the elderly and increased serum interleukin-6 in DAT. Aging (Milano). 1998 Aug; 10(4):316-23.

Bonaccorso S, Lin AH, Verkerk R, Van Hunsel F, Libbrecht I, Scharpe S, DeClerck L, Biondi M, Janca A, Maes M. Immune markers in fibromyalgia: comparison with major depressed patients and normal volunteers. J Affect Disord. $1998 \mathrm{Feb} ; 48(1): 75-82$.

Bonaccorso S, Maier SF, Meltzer HY, Maes M. Behavioral changes in rats after acute, chronic and repeated administration of interleukin-1beta: relevance for affective disorders. J Affect Disord. 2003 Nov; 77(2):143-8.

Bonaccorso S, Marino V, Biondi M, Grimaldi F, Ippoliti F, Maes M. Depression induced by treatment with interferon-alpha in patients affected by hepatitis C virus.J Affect Disord. 2002 Dec; 72(3):237-41.

Bonaccorso S, Marino V, Puzella A, Pasquini M, Biondi M, Artini M, Almerighi C, Verkerk R, Meltzer $\mathrm{H}$, Maes $M$. Increased depressive ratings in patients with hepatitis $C$ receiving interferon-alpha-based immunotherapy are related to interferon-alpha-induced changes in the serotonergic system.J Clin Psychopharmacol. 2002 Feb; 22(1):86-90. 
Bonaccorso S, Puzella A, Marino V, Pasquini $M$, Biondi $M$, Artini $M$, Almerighi $C$, Levrero $M$, Egyed B, Bosmans E, Meltzer HY, Maes M. Immunotherapy with interferon-alpha in patients affected by chronic hepatitis $C$ induces an intercorrelated stimulation of the cytokine network and an increase in depressive and anxiety symptoms. Psychiatry Res. 2001 Dec 15; 105(1-2):4555.

Bonaccorso S. Meltzer HY, Maes M. Psychological and behavioral effects of interferons. 2000. Current Opinion in Psychiatry. 13, 673-677.

Bonnet $M$, Lespinats $\mathrm{G}$, Burtin C. Evidence for serotonin ( $5 \mathrm{HT}$ ) binding sites on murine lymphocytes. Int J Immunopharmacol. 1987; 9(5):551-8.

Broderick PA. Interleukin 1alpha alters hippocampal serotonin and norepinephrine release during open-field behavior in Sprague-Dawley animals: differences from the Fawn-Hooded animal model of depression. Prog Neuropsychopharmacol Biol Psychiatry. 2002 Dec; 26(78):1355-72.

Brown KA. Factors modifying the migration of lymphocytes across the blood-brain barrier. Int Immunopharmacol. 2001 Nov; 1(12):2043-62.

Bunney WE Jr, Davis JM. Norepinephrine in depressive reactions. A review. Arch Gen Psychiatry. 1965 Dec; 13(6):483-94.

Buter J, de Vries EG, Sleijfer DT, Willemse PH, Mulder NH. Neuropsychiatric symptoms during treatment with interleukin-2. Lancet. 1993 Mar 6;341(8845):628.

Capuron L, Dantzer R. Cytokines and depression: the need for a new paradigm. Brain Behav Immun. 2003 Feb; 17 Suppl 1:S119-24.

Capuron L, Ravaud A, Neveu PJ, Miller AH, Maes M, Dantzer R. Association between decreased serum tryptophan concentrations and depressive symptoms in cancer patients undergoing cytokine therapy. Mol Psychiatry. 2002; 7(5):468-73.

Capuron L, Ravaud A. Prediction of the depressive effects of interferon alpha therapy by the patient's initial affective state. N Engl J Med. 1999 Apr 29; 340(17):1370.

Cassano WJ Jr, D'mello AP. Acute stress-induced facilitation of the hypothalamic-pituitaryadrenal axis: evidence for the roles of stressor duration and serotonin. Neuroendocrinology. 2001 Sep; 74(3):167-77.

Catalan R, Gallart JM, Castellanos JM, Galard R. Plasma corticotropin-releasing factor in depressive disorders. Biol Psychiatry. 1998 Jul 1; 44(1):15-20.

Cooper JR, Bloom FE, Roth RH: The Biochemical Basis of Neuropharmacology, Ed 3. Oxford University Press, New York, 1996.

Curzon G, Knott PJ. Rapid effects of environmental disturbance on rat plasma unesterified fatty acid and tryptophan concentrations and their prevention by antilipolytic drugs. $\mathrm{Br} J$ Pharmacol. 1975 Jul; 54(3):389-96.

Dafny N, Lee JR, Dougherty PM. Immune response products alter CNS activity: interferon modulates central opioid functions. J Neurosci Res. 1988; 19(1):130-9.

Dafny N, Prieto-Gomez B, Dong WQ, Reyes-Vazquez C. Interferon modulates neuronal activity recorded from the hypothalamus, thalamus, hippocampus, amygdala and the somatosensory cortex. Brain Res. 1996 Sep 23; 734(1-2):269-74.

Dantzer R. Cytokine-induced sickness behavior: where do we stand? Brain Behav Immun. 2001 Mar; 15(1):7-24.

David DJ, Renard CE, Jolliet P, Hascoet M, Bourin M. Antidepressant-like effects in various 
mice strains in the forced swimming test. Psychopharmacology (Berl). 2003 Apr; 166(4):373-82. Epub 2003 Feb 25.

de Beaurepaire R. Questions raised by the cytokine hypothesis of depression. Brain Behav Immun. 2002 Oct; 16(5):610-7.

Delgado PL, Charney DS, Price LH, Aghajanian GK, Landis H, Heninger GR. Serotonin function and the mechanism of antidepressant action. Reversal of antidepressant-induced remission by rapid depletion of plasma tryptophan. Arch Gen Psychiatry. 1990 May; 47(5):411-8.

Denicoff KD, Rubinow DR, Papa MZ, Simpson C, Seipp CA, Lotze MT, Chang AE, Rosenstein D, Rosenberg SA. The neuropsychiatric effects of treatment with interleukin-2 and lymphokineactivated killer cells. Ann Intern Med. 1987 Sep; 107(3):293-300.

Diagnostic Statistic Manual-IV-Text Revised (DSM-IV-TR). American Psychiatric Association. Masson, 2001.

Dinarello CA. The biology of interleukin 1 and comparison to tumor necrosis factor. Immunol Lett. 1987 Dec;16(3-4):227-31.

Dredge K, Connor TJ, Kelly JP, Leonard BE. Differential effect of a single high dose of the tricyclic antidepressant imipramine on interleukin-1beta and tumor necrosis factor-alpha secretion following an in vivo lipopolysaccharide challenge in rats. Int J Immunopharmacol. 1999 Oct; 21(10):663-73.

Dunn AJ, Berridge CW. Physiological and behavioral responses to corticotropin-releasing factor administration: is CRF a mediator of anxiety or stress responses? Brain Res Brain Res Rev. 1990 May-Aug; 15(2):71-100.

Dunn AJ, Chuluyan HE. Endotoxin elicits normal tryptophan and indolamine responses but impaired catecholamine and pituitary-adrenal responses in endotoxin-resistant mice. Life Sci. 1994; 54(13):847-53.

Dunn AJ, Welch J. Stress- and endotoxin-induced increases in brain tryptophan and serotonin metabolism depend on sympathetic nervous system activity. J Neurochem. 1991 Nov; 57(5):1615-22.

Dunn AJ. Systemic interleukin-1 administration stimulates hypothalamic norepinephrine metabolism parallelling the increased plasma corticosterone. Life Sci. 1988; 43(5):429-35.

Dursun SM, Blackburn JR, Kutcher SP. An exploratory approach to the serotonergic hypothesis of depression: bridging the synaptic gap. Med Hypotheses. 2001 Feb; 56(2):235-43.

Ericsson A, Kovacs KJ, Sawchenko PE. A functional anatomical analysis of central pathways subserving the effects of interleukin-1 on stress-related neuroendocrine neurons. J Neurosci. $1994 \mathrm{Feb} ; 14(2): 897-913$.

Fujino K, Yoshitake T, Inoue O, Ibii N, Kehr J, Ishida J, Nohta H, Yamaguchi M. Increased serotonin release in mice frontal cortex and hippocampus induced by acute physiological stressors. Neurosci Lett. 2002 Mar 1; 320(1-2):91-5.

Goldman LS. Successful treatment of interferon alpha-induced mood disorder with nortriptyline. Psychosomatics. 1994 Jul-Aug; 35(4):412-3.

Harrison BJ, Olver JS, Norman TR, Nathan PJ. Effects of serotonin and catecholamine depletion on interleukin- 6 activation and mood in human volunteers. Hum Psychopharmacol. 2002 Aug; 17(6):293-7.

Hayashi J, Kishihara Y, Yamaji K, Yoshimura E, Ohmiya M, Tani Y, Ikematsu H, Kashiwagi S. Serum levels of soluble interleukin-2 receptors and effects of interferon-alpha for patients 
with chronic hepatitis C virus. Dig Dis Sci. 1995 Aug; 4O(8):1837-41.

Hickey WF. Basic principles of immunological surveillance of the normal central nervous system. Glia. 2001 Nov; 36(2):118-24.

Higashi N, Nakamuta M, Motomura K, Enjoji M, Sakai H, Nawata H. Serum level of interleukin- 4 is a possible marker of therapeutic response to interferon treatment in patients with chronic hepatitis C. J Hepatol. 1999 Aug; 31(2):383.

Holsboer F: Neuroendocrinology of mood disorders. In Psychopharmacology: The Fourth Generation of Progress. FE Bloom, DJ Kupfer, editors. Raven Press, New York, 1995.

Ikegaya Y, Delcroix I, Iwakura Y, Matsuki N, Nishiyama N. Interleukin-1beta abrogates longterm depression of hippocampal CA1 synaptic transmission. Synapse. 2003 Jan; 47(1):54-7.

Jacobsen MB, Bratlie J, Reitan JB, Hellesnes J, Hanssen LE. Influence of interferon and radiation on serotonin content in primary carcinoid cell cultures. Acta Oncol. 1993; 32(2):239-43.

Janowsky DS, Risch SC, Ziegler MG, Gillin JC, Huey L, Rausch J. Physostigmine-induced epinephrine release in patients with affective disorder. Am J Psychiatry. 1986 Jul; 143(7):919-21.

Juengling FD, Ebert D, Gut O, Engelbrecht MA, Rasenack J, Nitzsche EU, Bauer J, Lieb K.Prefrontal cortical hypometabolism during low-dose interferon alpha treatment. Psychopharmacology (Berl). 2000 Nov; 152(4):383-9.

Kafka MS, Paul SM. Platelet alpha 2-adrenergic receptors in depression. Arch Gen Psychiatry. 1986 Jan; 43(1):91-5.

Kamata M, Higuchi H, Yoshimoto M, Yoshida K, Shimizu T. Effect of single intracerebroventricular injection of alpha-interferon on monoamine concentrations in the rat brain. Eur Neuropsychopharmacol. 2000 Mar; 10(2):129-32.

Kapur S, Mann JJ. Role of the dopaminergic system in depression. Biol Psychiatry. 1992 Jul 1; 32(1):1-17.

Kenis G, Maes M. Effects of antidepressants on the production of cytokines. Int J Neuropsychopharmacol. 2002 Dec; 5(4):401-12.

Kiecolt-Glaser JK, McGuire L, Robles TF, Glaser R. Emotions, morbidity, and mortality: new perspectives from psychoneuroimmunology. Annu Rev Psychol. 2002; 53:83-107.

Kirkwood JM, Bender C, Agarwala S, Tarhini A, Shipe-Spotloe J, Smelko B, Donnelly S, Stover L. Mechanisms and management of toxicities associated with high-dose interferon alfa-2b therapy. J Clin Oncol. 2002 Sep 1; 20(17):3703-18.

Kitayama I, Cintra A, Janson AM, Fuxe K, Agnati LF, Eneroth P, Aronsson M, Harfstrand A, Steinbush HW, Visser TJ, et al. Chronic immobilization stress: evidence for decreases of 5hydroxy-tryptamine immunoreactivity and for increases of glucocorticoid receptor immunoreactivity in various brain regions of the male rat. J Neural Transm. 1989; 77(2-3):93130.

Konsman JP, Parnet P, Dantzer R. Cytokine-induced sickness behaviour: mechanisms and implications. Trends Neurosci. 2002 Mar; 25(3):154-9.

Kugaya A, Kagaya A, Uchitomi Y, Yokota N, Yamawaki S. Effect of interferon-alpha on DOIinduced wet-dog shakes in rats. J Neural Transm. 1996; 103(8-9):947-55.

Lacosta S, Merali Z, Anisman H. Influence of interleukin-1beta on exploratory behaviors, plasma ACTH, corticosterone, and central biogenic amines in mice. Psychopharmacology (Berl). 1998 Jun; 137(4):351-61.

Lesch KP, Bengel D, Heils A, Sabol SZ, Greenberg BD, Petri S, Benjamin J, Muller CR, Hamer DH, 
Murphy DL. Association of anxiety-related traits with a polymorphism in the serotonin transporter gene regulatory region. Science. 1996 Nov 29; 274(5292):1527-31.

Lesch KP, Mossner R. Genetically driven variation in serotonin uptake: is there a link to affective spectrum, neurodevelopmental, and neurodegenerative disorders? Biol Psychiatry. 1998 Aug 1; 44(3):179-92.

Lieberman AP, Pitha PM, Shin HS, Shin ML. Production of tumor necrosis factor and other cytokines by astrocytes stimulated with lipopolysaccharide or a neurotropic virus. Proc Natl Acad Sci U S A. 1989 Aug; 86(16):6348-52.

Linthorst AC, Flachskamm C, Hopkins SJ, Hoadley ME, Labeur MS, Holsboer F, Reul JM. Longterm intracerebroventricular infusion of corticotropin-releasing hormone alters neuroendocrine, neurochemical, autonomic, behavioral, and cytokine responses to a systemic inflammatory challenge. J Neurosci. 1997 Jun 1; 17(11):4448-6o.

Linthorst AC, Flachskamm C, Muller-Preuss P, Holsboer F, Reul JM. Effect of bacterial endotoxin and interleukin-1 beta on hippocampal serotonergic neurotransmission, behavioral activity, and free corticosterone levels: an in vivo microdialysis study. J Neurosci. 1995 Apr; 15(4):2920-34.

Madden KS, Livnat S. "Catecholamine Action and Immunologic Reactivity" in Ader R., Felten DL, Cohen N (eds), Psychoneuroimmunology, San Diego, Academic Press Inc. 1991.

Maddrey WC. Safety of combination interferon alfa-2b/ribavirin therapy in chronic hepatitis C-relapsed and treatment-naive patients. Semin Liver Dis. 1999; 19 Suppl 1:67-75.

Maes M, Bosmans E, Suy E, Minner B, Raus J. A further exploration of the relationships between immune parameters and the HPA-axis activity in depressed patients. Psychol Med. 1991 May; 21(2):313-20.

Maes M, Bosmans E, Suy E, Vandervorst C, De Jonckheere C, Raus J. Immune disturbances during major depression: upregulated expression of interleukin-2 receptors. Neuropsychobiology. 1990-91; 24(3):115-20.

Maes M, Lin A, Bosmans E, Vandoolaeghe E, Bonaccorso S, Kenis G, De Jongh R, Verkerk R, Song $C$, Scharpe S, Neels $H$. Serotonin-immune interactions in detoxified chronic alcoholic patients without apparent liver disease: activation of the inflammatory response system and lower plasma total tryptophan. Psychiatry Res. 1998 May 8; 78(3):151-61.

Maes M, Meltzer HY, Scharpe S, Bosmans E, Suy E, De Meester I, Calabrese J, Cosyns P. Relationships between lower plasma L-tryptophan levels and immune-inflammatory variables in depression. Psychiatry Res. 1993 Nov; 49(2):151-65.

Maes M, Meltzer HYM. The serotonin hypothesis of major depression. In: Bloom FE, Kupfer DJ, Eds. Psychopharmacology the Fourth Generation of Progress. Raven Press, New York, 1995:933-944.

Maes M, Minner B, Suy E, Vandervorst C, Raus J. Coexisting dysregulations of both the sympathoadrenal system and hypothalamic-pituitary-adrenal-axis in melancholia. J Neural Transm Gen Sect. 1991; 85(3):195-210.

Maes M, Scharpe S, Meltzer HY, Bosmans E, Suy E, Calabrese J, Cosyns P. Relationships between interleukin- 6 activity, acute phase proteins, and function of the hypothalamic-pituitary-adrenal axis in severe depression. Psychiatry Res. 1993 Oct; 49(1):11-27.

Maes M, Scharpe S, Meltzer HY, Okayli G, Bosmans E, D’Hondt P, Vanden Bossche BV, Cosyns $P$. Increased neopterin and interferon-gamma secretion and lower availability of L-tryptophan 
in major depression: further evidence for an immune response. Psychiatry Res. 1994 Nov; 54(2):143-60.

Maes M, Smith R, Scharpe S. The monocyte-T-lymphocyte hypothesis of major depression. Psychoneuroendocrinology. 1995; 20(2):111-6.

Maes M, Song C, Lin AH, Bonaccorso S, Kenis G, De Jongh R, Bosmans E, Scharpe S. Negative immunoregulatory effects of antidepressants: inhibition of interferon-gamma and stimulation of interleukin-10 secretion. Neuropsychopharmacology. 1999 Apr; 20(4):370-9.

Maes M, Van Gastel A, Delmeire L, Meltzer HY. Decreased platelet alpha-2 adrenoceptor density in major depression: effects of tricyclic antidepressants and fluoxetine. Biol Psychiatry. 1999 Feb 1; 45(3):278-84.

Maes M, Wauters A, Verkerk R, Demedts P, Neels H, Van Gastel A, Cosyns P, Scharpe S, Desnyder R. Lower serum L-tryptophan availability in depression as a marker of a more generalized disorder in protein metabolism. Neuropsychopharmacology. 1996 Sep; 15(3):243-51.

Maes M. 1997. The immune pathophysiology of major depression. In Depression: Neurobiological, Psychopathological and Therapeutic Advances. Honig A, van Praag H (eds). John Wiley: Chichester; 197-215.

Maes M. A review on the acute phase response in major depression. Rev Neurosci. 1993 OctDec; 4(4):407-16.

Maes M. Interleukin-1beta and the etiology of depression. Acta Psychiatr Scand. 2001 Mar; 103(3):161-2.

Maes M. Major depression and activation of the inflammatory response system. Adv Exp Med Biol. 1999; 461:25-46.

Maier SF, Goehler LE, Fleshner M, Watkins LR. The role of the vagus nerve in cytokine-tobrain communication. Ann N Y Acad Sci. 1998 May 1; 840:289-300.

Maier SF, Watkins LR. Intracerebroventricular interleukin-1 receptor antagonist blocks the enhancement of fear conditioning and interference with escape produced by inescapable shock. Brain Res. 1995 Oct 16; 695(2):279-82.

Maier SF, Wiertelak EP, Martin D, Watkins LR. Interleukin-1 mediates the behavioral hyperalgesia produced by lithium chloride and endotoxin. Brain Res. 1993 Oct 1; 623(2):321-4.

Makino M, Kitano Y, Komiyama C, Hirohashi M, Kohno M, Moriyama M, Takasuna K. Human interferon-alpha induces immobility in the mouse forced swimming test: involvement of the opioid system. Brain Res. 2000 Jan 10; 852(2):482-4.

Malik UR, Makower DF, Wadler S. Interferon-mediated fatigue.

Cancer. 2001 Sep 15; 92 (6 Suppl):1664-8.

Manfridi A., Brambilla D., Imeri L. Interleukin-1beta inhibits serotonergic raphe neurons in guinea pig brain slices. Soc. Neurosci. Abstr. 2001; 28, 324.

Mansbach RS, Brooks EN, Chen YL. Antidepressant-like effects of CP-154,526, a selective CRF1 receptor antagonist. Eur J Pharmacol. 1997 Mar 26; 323(1):21-6.

Martelletti P, Alteri E, Pesce A, Rinaldi-Garaci C, Giacovazzo M. Defect of serotonin binding to mononuclear cells from episodic cluster headache patients. Headache. 1987 Jan; 27(1):23-6.

Matta S, Singh J, Newton R, Sharp BM. The adrenocorticotropin response to interleukin-1 beta instilled into the rat median eminence depends on the local release of catecholamines. Endocrinology. 1990 Nov; 127(5):2175-82.

Mayberg HS. Limbic-cortical dysregulation: a proposed model of depression. J 
Neuropsychiatry Clin Neurosci. 1997 Summer; 9(3):471-81.

McGeer PL, McGeer EG. The inflammatory response system of brain: implications for therapy of Alzheimer and other neurodegenerative diseases. Brain Res Brain Res Rev. 1995 Sep; 21(2):195-218.

Menzies RA, Patel R, Hall NR, O'Grady MP, Rier SE. Human recombinant interferon alpha inhibits naloxone binding to rat brain membranes. Life Sci. 1992; 50(24):PL227-32.

Merali Z, Brennan K, Brau P, Anisman H. Dissociating anorexia and anhedonia elicited by interleukin-1beta: antidepressant and gender effects on responding for "free chow" and "earned" sucrose intake. Psychopharmacology (Berl). 2003 Feb; 165(4):413-8. Epub 2002 Nov 30.

Miller A. H., Pearce B., Pariante C.M. Immune System and the Central Nervous System Interaction In: Kaplan Sadock's Comphrensive Textbook of Psychiatry. Seventh Edition on CDROM, 2000, Lippincott Williams and Wilkins.

Mitchell AJ. The role of corticotropin releasing factor in depressive illness: a critical review. Neurosci Biobehav Rev. 1998 Sep; 22(5):635-51.

MohanKumar PS, Quadri SK. Systemic administration of interleukin-1 stimulates norepinephrine release in the paraventricular nucleus. Life Sci. 1993; 52(24):1961-7.

MohanKumar SM, MohanKumar PS, Quadri SK. Specificity of interleukin-1beta-induced changes in monoamine concentrations in hypothalamic nuclei: blockade by interleukin-1 receptor antagonist. Brain Res Bull. 1998 Sep 1; 47(1):29-34.

Morikawa O, Sakai N, Obara H, Saito N. Effects of interferon-alpha, interferon-gamma and CAMP on the transcriptional regulation of the serotonin transporter. Eur J Pharmacol. 1998 May 22; 349(2-3):317-24.

Nakamori T, Morimoto A, Yamaguchi K, Watanabe T, Long NC, Murakami N. Organum vasculosum laminae terminalis (OVLT) is a brain site to produce interleukin-1 beta during fever. Brain Res. 1993 Jul 30; 618(1):155-9.

Naveau S, Balian A, Degos F, Daurat V, Chevret S, Gayno S, Bastie A, Riachi G, BartolomeiPortal I, Barange K, Moussalli J, Bailly F, Chaumet-Riffaud P, Emilie D. Prognostic value of the soluble interleukin-2 receptor in chronic hepatitis $C$ treated with interferon-alfa. Multicenter GER-CYT O4 Group. J Hepatol. 1999 Oct; 31(4):612-7.

Norman SE, Chediak AD, Freeman C, Kiel M, Mendez A, Duncan R, Simoneau J, Nolan B. Sleep disturbances in men with asymptomatic human immunodeficiency (HIV) infection. Sleep. $1992 \mathrm{Apr} ; 15(2): 150-5$.

Owen BM, Eccleston D, Ferrier IN, Young AH. Raised levels of plasma interleukin-1beta in major and postviral depression. Acta Psychiatr Scand. 2001 Mar; 103(3):226-8.

Owens MJ, Nemeroff CB. Physiology and pharmacology of corticotropin-releasing factor. Pharmacol Rev. 1991 Dec; 43(4):425-73.

Pan W, Banks WA, Kastin AJ. Permeability of the blood-brain and blood-spinal cord barriers to interferons. J Neuroimmunol. 1997 Jun; 76(1-2):105-11.

Pollmacher T, Haack M, Schuld A, Reichenberg A, Yirmiya R. Low levels of circulating inflammatory cytokines-do they affect human brain functions? Brain Behav Immun. 2002 Oct; 16(5):525-32.

Raber J, Koob GF, Bloom FE. Interferon-alpha and transforming growth factor-beta 1 regulate corticotropin-releasing factor release from the amygdala: comparison with the hypothalamic 
response. Neurochem Int. 1997 Apr-May; 30(4-5):455-63.

Raivich G, Jones LL, Kloss CU, Werner A, Neumann H, Kreutzberg GW. Immune surveillance in the injured nervous system: T-lymphocytes invade the axotomized mouse facial motor nucleus and aggregate around sites of neuronal degeneration. J Neurosci. 1998 Aug 1; 18(15):5804-16.

Recht $\mathrm{M}$, Borden $\mathrm{EC}$, Knight $\mathrm{E}$ Jr. A human 15-kDa IFN-induced protein induces the secretion of IFN-gamma. J Immunol. 1991 Oct 15; 147(8):2617-23.

Rief W, Pilger F, Ihle D, Bosmans E, Egyed B, Maes M. Immunological differences between patients with major depression and somatization syndrome. Psychiatry Res. 2001 Dec 31; 105(3):165-74.

Rosenstein DL, Lerner D, Cai J. More on the depressive effects of interferon alpha. N Engl J Med. 1999 Sep 9; 341(11):849-50.

Sakic B, Szechtman H, Braciak T, Richards C, Gauldie J, Denburg JA. Reduced preference for sucrose in autoimmune mice: a possible role of interleukin-6. Brain Res Bull. 1997; 44(2):15565.

Sammut S, Bethus I, Goodall G, Muscat R. Antidepressant reversal of interferon-alphainduced anhedonia. Physiol Behav. 2002 Apr 15; 75(5):765-72.

Sargent PA, Kjaer KH, Bench CJ, Rabiner EA, Messa C, Meyer J, Gunn RN, Grasby PM, Cowen PJ. Brain serotonin1A receptor binding measured by positron emission tomography with [11C]WAY-100635: effects of depression and antidepressant treatment. Arch Gen Psychiatry. $2000 \mathrm{Feb} ;$ 57(2): 174-80.

Shuto H, Kataoka Y, Horikawa T, Fujihara N, Oishi R. Repeated interferon-alpha administration inhibits dopaminergic neural activity in the mouse brain. Brain Res. 1997 Feb 7; 747(2):34851.

Silverman DH, Krueger JM, Karnovsky ML. Specific binding sites for muramyl peptides on murine macrophages. J Immunol. 1986 Mar 15; 136(6):2195-201.

Sluzewska A, Rybakowski JK, Laciak M, Mackiewicz A, Sobieska M, Wiktorowicz K. Interleukin- 6 serum levels in depressed patients before and after treatment with fluoxetine. Ann N Y Acad Sci. 1995 Jul 21;762:474-6.

Sluzewska A, Rybakowski JK, Sobieska M, Wiktorowicz K. Concentration and microheterogeneity glycophorms of alpha-1-acid glycoprotein in major depressive disorder. J Affect Disord. 1996 Jul 8; 39(2):149-55.

Smith RS. The macrophage theory of depression. Med Hypotheses. 1991 Aug; 35(4):298-306.

Song C, Dinan T, Leonard BE. Changes in immunoglobulin, complement and acute phase protein levels in the depressed patients and normal controls. J Affect Disord. 1994 Apr; 30(4):283-8.

Song C, Lin A, Bonaccorso S, Heide C, Verkerk R, Kenis G, Bosmans E, Scharpe S, Whelan A, Cosyns $P$, de Jongh $R$, Maes $M$. The inflammatory response system and the availability of plasma tryptophan in patients with primary sleep disorders and major depression. J Affect Disord. 1998 Jun; 49(3):211-9.

Song $\mathrm{C}$. The interaction between cytokines and neurotransmitters in depression and stress: possible mechanism of antidepressant treatments. Hum Psychopharmacol. 2000 Apr; 15(3):199-211.

Spath-Schwalbe E, Hansen K, Schmidt F, Schrezenmeier H, Marshall L, Burger K, Fehm HL, 
Born J. Acute effects of recombinant human interleukin- 6 on endocrine and central nervous sleep functions in healthy men. J Clin Endocrinol Metab. 1998 May; 83(5):1573-9.

Stastny J, Konstantinidis A, Schwarz MJ, Rosenthal NE, Vitouch O, Kasper S, Neumeister A. Effects of tryptophan depletion and catecholamine depletion on immune parameters in patients with seasonal affective disorder in remission with light therapy. Biol Psychiatry. 2003 Feb 15; 53(4):332-7.

Stitt JT, Bernheim HA. Differences in endogenous pyrogen fevers induced by iv and icv routes in rabbits. J Appl Physiol. 1985 Aug; 59(2):342-7.

Sun D, Whitaker JN, Wilson DB. Regulatory $T$ cells in experimental allergic encephalomyelitis. III. Comparison of disease resistance in Lewis and Fischer 344 rats. Eur J Immunol. 1999 Apr; 29(4):1101-6.

Sweep CG, van der Meer MJ, Hermus AR, Smals AG, van der Meer JW, Pesman GJ, Willemsen SJ, Benraad TJ, Kloppenborg PW. Chronic stimulation of the pituitary-adrenal axis in rats by interleukin-1 beta infusion: in vivo and in vitro studies. Endocrinology. 1992 Mar; 130(3):115364.

Taylor MW, Feng GS. Relationship between interferon-gamma, indoleamine 2, 3-dioxygenase, and tryptophan catabolism. FASEB J. 1991 Aug;5(11):2516-22.

Thase M.E. Mood Disorders: Neurobiology. In: Kaplan Sadock's Comphrensive Textbook of Psychiatry. Seventh Edition on CD-ROM, 2000, Lippincott Williams and Wilkins.

Tipold A, Vandevelde M, Wittek R, Moore P, Summerfield A, Zurbriggen A. Partial protection and intrathecal invasion of CD8(+) T cells in acute canine distemper virus infection. Vet Microbiol. 2001 Nov 26; 83(3):189-203.

Titinchi S, Clark B. Alpha 2-adrenoceptors in human lymphocytes: direct characterisation by [3H] yohimbine binding. Biochem Biophys Res Commun. 1984 May 31; 121(1):1-7.

Vgontzas AN, Papanicolaou DA, Bixler EO, Kales A, Tyson K, Chrousos GP. Elevation of plasma cytokines in disorders of excessive daytime sleepiness: role of sleep disturbance and obesity. J Clin Endocrinol Metab. 1997 May; 82(5):1313-6.

Watkins LR, Goehler LE, Relton JK, Tartaglia N, Silbert L, Martin D, Maier SF. Blockade of interleukin-1 induced hyperthermia by subdiaphragmatic vagotomy: evidence for vagal mediation of immune-brain communication. Neurosci Lett. 1995 Jan 2; 183(1-2):27-31.

Westly HJ, Kelley KW. Down-regulation of glucocorticoid and beta-adrenergic receptors on lectin-stimulated splenocytes. Proc Soc Exp Biol Med. 1987 Jun; 185(2):211-8.

Wichers $M$, Maes $M$. The psychoneuroimmuno-pathophysiology of cytokine-induced depression in humans. Int J Neuropsychopharmacol. 2002 Dec; 5(4):375-88.

Widner B, Sepp N, Kowald E, Ortner U, Wirleitner B, Fritsch P, Baier-Bitterlich G, Fuchs D. Enhanced tryptophan degradation in systemic lupus erythematosus. Immunobiology. 2000 Apr; 201(5):621-30.

Yamada T, Yamanaka I. Microglial localization of alpha-interferon receptor in human brain tissues. Neurosci Lett. 1995 Apr 14; 189(2):73-6.

Yamano M, Yuki H, Yasuda S, Miyata K. Corticotropin-releasing hormone receptors mediate consensus interferon-alpha YM643-induced depression-like behavior in mice.J Pharmacol Exp Ther. 2000 Jan; 292(1):181-7.

Yatham LN, Liddle PF, Shiah IS, Scarrow G, Lam RW, Adam MJ, Zis AP, Ruth TJ. Brain serotonin2 receptors in major depression: a positron emission tomography study. Arch Gen Psychiatry. 
2000 Sep; 57(9):850-8.

Yuwiler A, Oldendorf WH, Geller E, Braun L. Effect of albumin binding and amino acid competition on tryptophan uptake into brain. J Neurochem. 1977 May; 28(5):1015-23.

Zalcman S, Green-Johnson JM, Murray L, Nance DM, Dyck D, Anisman H, Greenberg AH. Cytokine-specific central monoamine alterations induced by interleukin-1, -2 and -6 . Brain Res. 1994 Apr 18; 643(1-2):40-9.

Zubareva OE, Krasnova IN, Abdurasulova IN, Bluthe RM, Dantzer R, Klimenko VM. Effects of serotonin synthesis blockade on interleukin-1 beta action in the brain of rats. Brain Res. 2001 Oct 12; 915(2):244-7. 